Supporting Information for

\title{
Highly Distorted Chiral Two-Dimensional Tin Iodide Perovskites for Spin Polarized Charge Transport
}

Haipeng Lu, ${ }^{1}$ Chuanxiao Xiao, ${ }^{1}$ Ruyi Song, ${ }^{2}$ Tianyang Li,${ }^{2}$ Annalise E. Maughan, ${ }^{1}$ Andrew Levin, ${ }^{1}$ Roman Brunecky, ${ }^{3}$ Joseph J. Berry, ${ }^{1}$ David B. Mitzi, ${ }^{2}$ Volker Blum, ${ }^{2}$ and Matthew C. Beard ${ }^{*} 1$

${ }^{1}$ Chemistry \& Nanoscience Center, National Renewable Energy Laboratory, Golden, Colorado 80401, United States

${ }^{2}$ Department of Mechanical Engineering and Material Science, Duke University, Durham, North Carolina 27708, United States

${ }^{3}$ Chemical and Bioscience Center, National Renewable Energy Laboratory, Golden, Colorado 80401, United States

\section{Corresponding Author}

Matthew C. Beard*: Matt.Beard@nrel.gov

\section{Table of Contents}

I. Distortion index and bond angle variance of $(R-/ S \text { - } / \text { rac-MBA })_{2} \mathrm{SnI}_{4}$ compounds

II. Structural and bonding parameters of $(R-/ S-/ r a c-M B A)_{2} \mathrm{SnI}_{4}$ and $(R-\mathrm{MBA})_{2} \mathrm{PbI}_{4}$ compounds

III. Comparison of the in-plane lattice parameters between representative layered Sn-I and $\mathrm{Pb}-\mathrm{I}$ perovskites

IV. Generalized Elliot Equation

V. EDS results

VI. Powder-XRD data and Pawley refinements

VII. Additional details of theoretical simulation

VIII. AFM images and magnetic CP-AFM data

IX. Geometry information for electronic structure calculations 
I. Distortion index and bond angle variance of $(R-/ S \text { - } / \text { rac-MBA })_{2} \mathrm{SnI}_{4}$ compounds

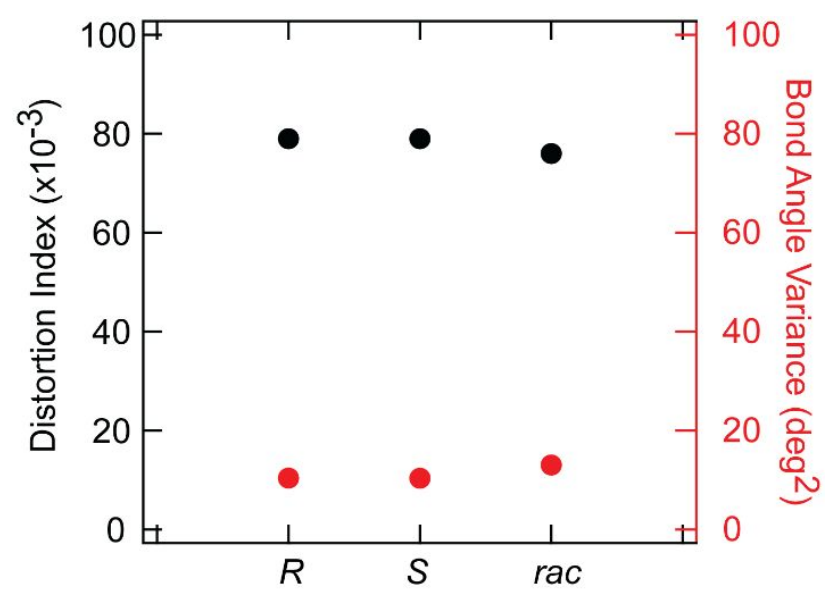

Figure S1. Bond length distortion index (D) and bond angle variance $\left(\mathrm{deg}^{2}\right)$ of $(R-\mathrm{MBA})_{2} \mathrm{SnI}_{4},(S-$ $\mathrm{MBA})_{2} \mathrm{SnI}_{4}$, and ( $\left.\mathrm{rac}-\mathrm{MBA}\right)_{2} \mathrm{SnI}_{4}$ compounds calculated based on the crystal structures.

\section{Structural and bonding parameters of $(R-/ S-/ r a c-M B A){ }_{2} \mathrm{SnI}_{4}$ and $(R-M B A)_{2} \mathrm{PbI}_{4}$ compounds}

Table S1. Comparison of the structural and bonding parameters between $(R-\mathrm{MBA})_{2} \mathrm{SnI}_{4},(S$ $\mathrm{MBA})_{2} \mathrm{SnI}_{4},(\mathrm{rac}-\mathrm{MBA})_{2} \mathrm{SnI}_{4}$, and $(R-\mathrm{MBA})_{2} \mathrm{PbI}_{4}$.

$$
(R-\mathrm{MBA})_{2} \mathrm{SnI}_{4} \quad(S-\mathrm{MBA})_{2} \mathrm{SnI}_{4} \quad(\text { rac-MBA })_{2} \mathrm{SnI}_{4} \quad(R-\mathrm{MBA})_{2} \mathrm{PbI}_{4}{ }^{1}
$$

\begin{tabular}{lcccc}
\hline $\begin{array}{l}\text { Average M-I bond } \\
\text { length }(\AA)\end{array}$ & 3.267 & 3.267 & 3.261 & 3.258 \\
\hline $\begin{array}{l}\text { Octahedra } \\
\text { distortion index (D) }\end{array}$ & 0.079 & 0.079 & 0.076 & 0.021 \\
\hline $\begin{array}{l}\text { Quadratic } \\
\text { elongation }(\lambda)\end{array}$ & 1.0112 & 1.0113 & 1.0115 & 1.0058 \\
\hline $\begin{array}{l}\text { Average M-I-M } \\
\text { bond angle (deg) }\end{array}$ & 153.383 & 153.389 & 153.634 & 150.78 \\
\hline $\begin{array}{l}\text { Average bond angle } \\
\text { variance }\left(\mathrm{deg}^{2}\right)\end{array}$ & 10.3754 & 10.3507 & 13.0358 & 18.2869 \\
\hline
\end{tabular}




\section{Comparison of the in-plane lattice parameters between representative layered Sn-I and $\mathrm{Pb}-\mathrm{I}$ perovskites}

Table S2. Comparison of the in-plane lattice parameters between representative layered Sn-I and $\mathrm{Pb}-\mathrm{I}$ perovskites templated by the same organic cations based on literature reports.

\begin{tabular}{|c|c|c|c|}
\hline Organic Cations (L) & Space group & $(\mathrm{L})_{2} \mathrm{PbI}_{4}$ & $(\mathrm{~L})_{2} \mathrm{SnI}_{4}$ \\
\hline Phenethylammonium (PEA) ${ }^{2,3}$ & $C 2 / m$ & $\begin{array}{l}b=6.131 \AA \\
c=6.185 \AA\end{array}$ & $\begin{array}{l}b=6.117 \AA \\
c=6.164 \AA\end{array}$ \\
\hline Butylammonium $(\mathrm{BA})^{4}$ & Pbca & $\begin{array}{l}a=8.8632 \AA \\
b=8.6816 \AA\end{array}$ & $\begin{array}{l}a=8.8370 \AA \\
b=8.6191 \AA\end{array}$ \\
\hline Benzylammonium (BZA) ${ }^{5}$ & $P 2_{1} / n$ & $\begin{array}{l}a=8.9159 \AA \\
c=8.9925 \AA\end{array}$ & $\begin{array}{l}a=8.7411 \AA \\
c=8.9845 \AA\end{array}$ \\
\hline Histammonium $(\mathrm{HA})^{5}$ & Pbca & $\begin{array}{l}a=9.1561 \AA \\
b=8.6894 \AA\end{array}$ & $\begin{array}{l}a=9.0944 \AA \\
b=8.6613 \AA\end{array}$ \\
\hline
\end{tabular}

* We can see that, when templated with these organic cationas, PEA, BA, BZA, and HA, the inplane lattice parameters are all larger in $\mathrm{Pb}$-I layered perovskites versus those in layered $\mathrm{Sn}$-I perovskites. 


\section{Generalized Elliot Equation.}

$\alpha(h v)$

$$
\begin{aligned}
& =\frac{A \quad \Gamma\left(E_{e x}\right)}{\frac{\zeta \Gamma\left(E_{e x}\right)^{2}}{4}+\left[\frac{h v-E_{e x}}{\zeta}\right]^{2}}+B \frac{1}{1+\theta\left(h v-E_{g}\right) \exp \left\{-2 \pi\left[\frac{h v-E_{g}}{R y}\right]^{-1 / 2}\right\}} \\
& \left\{\frac{1}{2}+\frac{1}{\pi} \arctan \left[\frac{2\left(h v-E_{g}\right)}{\Gamma\left(E_{g}\right)}\right]\right\}
\end{aligned}
$$

where, $E_{e x}=E_{g}-R y, \zeta$ is a broadening parameter, $\mathrm{A}$ and $\mathrm{B}$ are the relative amplitudes of the exciton and free carrier absorptions and

$$
\Gamma\left(E_{x}\right)=\frac{2\left(\Gamma_{0}\right)}{\exp \left[-3\left(h v-E_{x}\right) / k_{B} T\right]+1}
$$

\section{EDS results}

Table S3. Summary of EDS results based on freshly-synthesized crystals

\begin{tabular}{lccc} 
Theoretical Formula & Pb atomic \% & Sn atomic \% & I atomic \% \\
\hline$(R \text {-MBA })_{2} \mathrm{PbI}_{4}$ & 20.15 & 0 & 79.85 \\
\hline$(R-\mathrm{MBA})_{2} \mathrm{~Pb}_{0.95} \mathrm{Sn}_{0.05} \mathrm{I}_{4}$ & 14.57 & 1.86 & 83.57 \\
\hline$(R-\mathrm{MBA})_{2} \mathrm{~Pb}_{0.8} \mathrm{Sn}_{0.2} \mathrm{I}_{4}$ & 11.41 & 5.17 & 83.42 \\
\hline$(R-\mathrm{MBA})_{2} \mathrm{~Pb}_{0.5} \mathrm{Sn}_{0.5} \mathrm{I}_{4}$ & 10.02 & 9.09 & 80.89 \\
\hline$(R-\mathrm{MBA})_{2} \mathrm{~Pb}_{0.20} \mathrm{Sn}_{0.80} \mathrm{I}_{4}$ & 2.42 & 17.11 & 80.47 \\
\hline$(R-\mathrm{MBA})_{2} \mathrm{SnI}_{4}$ & 0 & 23.42 & 75.58 \\
\hline
\end{tabular}

* Here we only include atomic ratio of $\mathrm{Sn}, \mathrm{Pb}$ and I, excluding $\mathrm{C}, \mathrm{N}$, and $\mathrm{H}$. 
VI. Powder-XRD data and Pawley refinements 

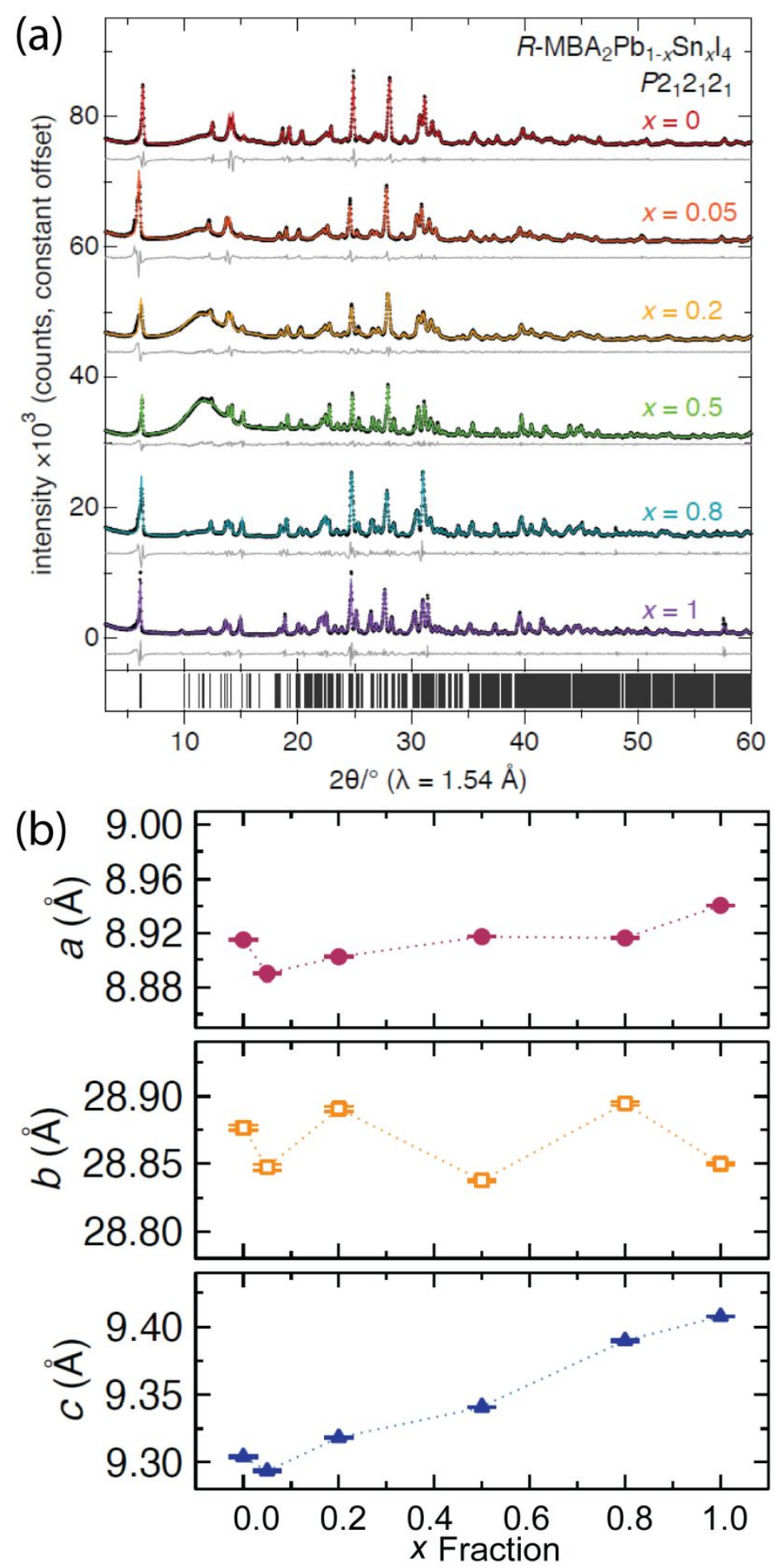

Figure S2. Pawley refinements of the orthorhombic structures (space group $P 2_{1} 2_{1} 2_{1}$ ) of $R$ $\mathrm{MBA}_{2} \mathrm{~Pb}_{1-x} \mathrm{Sn}_{x} \mathrm{I}_{4}$ from laboratory powder X-ray diffraction data (a) and extracted lattice parameters (b). Black circles represent the data, colored lines represent the fits, and light grey lines represent the difference curves. The vertical tick marks in the subplot represent the position of predicted reflections for the orthorhombic structure determined by single crystal X-ray diffraction of $R$ - 
$\mathrm{MBA}_{2} \mathrm{SnI}_{4}$. The amorphous background in powder XRD is due to glass substrate. Pawley refinement results show that the in-plane lattice parameters ( $a$ and $c$ ) increase with increasing tin content.

Table S4. Fitted structural and refinement parameters for Pawley refinements of the powder Xray diffraction data of the $(R-\mathrm{MBA})_{2} \mathrm{~Pb}_{1-x} \mathrm{Sn}_{x} \mathrm{I}_{4}$ series. Errors are denoted in the parentheses.

\begin{tabular}{lllll}
\hline$x$ in $\mathrm{MBA}_{2} \mathrm{~Pb}_{1-x} \mathrm{Sn}_{x} \mathrm{I}_{4}$ & $a(\AA)$ & $b(\AA)$ & $c(\AA)$ & $R_{w p}(\%)$ \\
\hline 0 & $8.9148(7)$ & $28.877(2)$ & $9.3041(8)$ & 9.85 \\
0.05 & $8.8898(8)$ & $28.847(2)$ & $9.293(1)$ & 8.49 \\
0.2 & $8.9021(8)$ & $28.891(2)$ & $9.3184(9)$ & 7.47 \\
0.5 & $8.9171(3)$ & $28.8374(9)$ & $9.3409(3)$ & 5.44 \\
0.8 & $8.9161(7)$ & $28.895(1)$ & $9.3900(7)$ & 12.05 \\
1 & $8.9404(4)$ & $28.850(1)$ & $9.4080(5)$ & 11.04 \\
\hline
\end{tabular}




\section{Additional details of computational simulations}

(a)

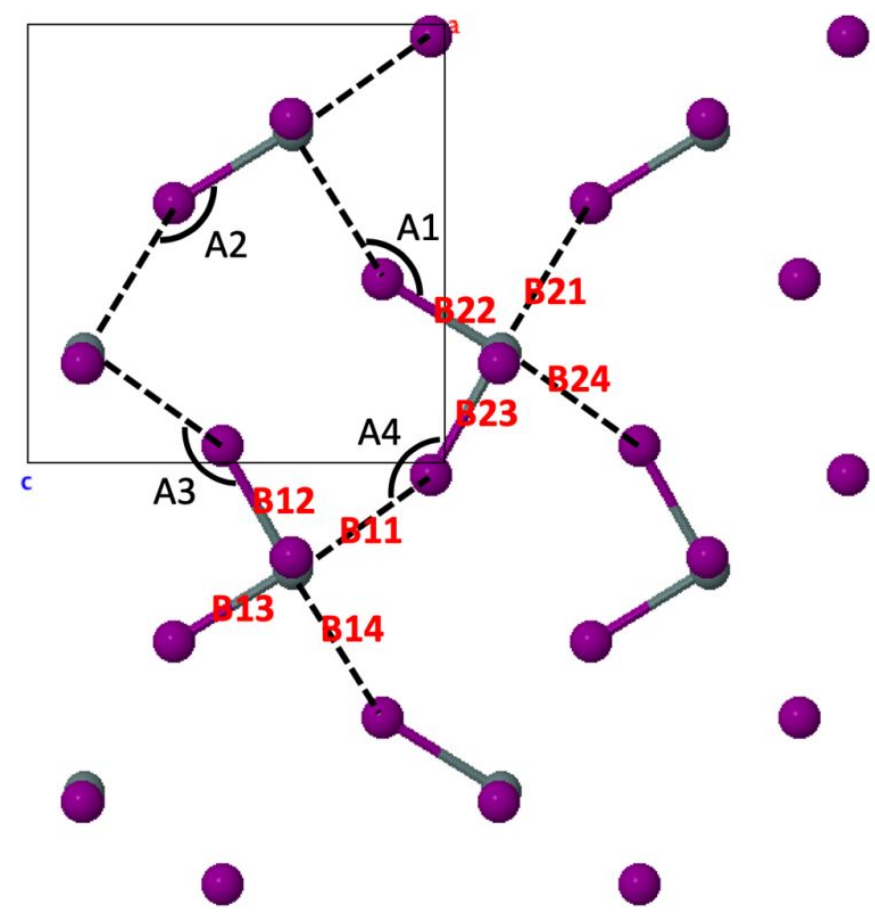

(b)

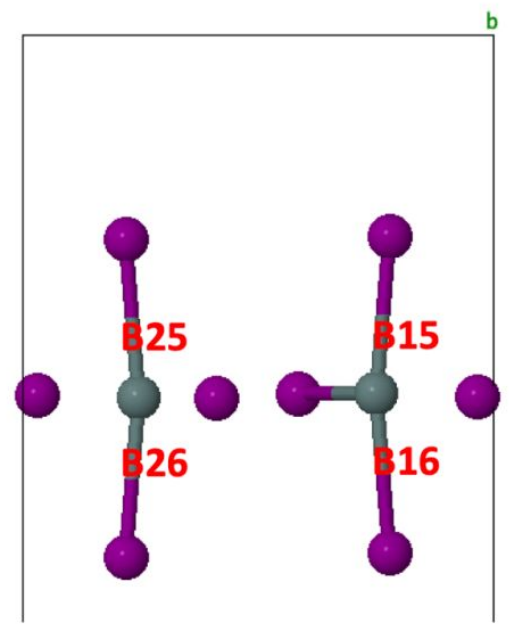

Figure S3. Illustration of the bond length and bond angle labels used in geometry comparisons between input and relaxed structures for $(S-\mathrm{MBA})_{2} \mathrm{~Pb}_{1-x} \mathrm{Sn}_{x} \mathrm{I}_{4}(x=0,0.5,1)$. This in-plane unit cell is denoted $\mathrm{c}(2 \times 2)$. The $(S-\mathrm{MBA})_{2} \mathrm{SnI}_{4}$ structure is here shown as an example. (a) Top view of the inorganic layer along $b$-axis. (b) Side view of the inorganic layer along $a$-axis. The labels of bond angles are colored in black and bond lengths are colored in red. 
(a)

(b)

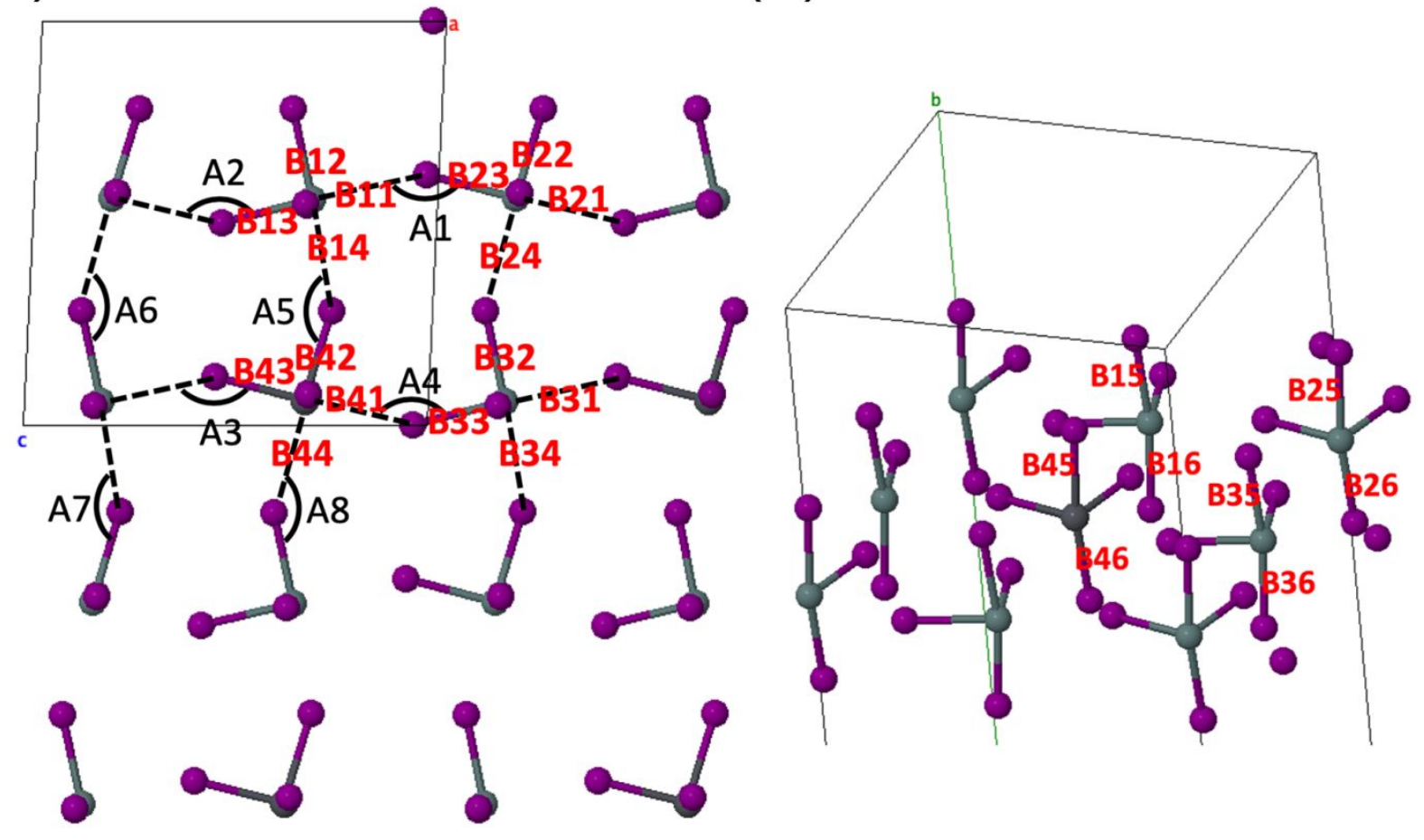

Figure S4. Illustration of the bond length and bond angle labels used in geometry comparison between input and relaxed structures for $(S-\mathrm{MBA})_{2} \mathrm{~Pb}_{1-x} \mathrm{Sn}_{x} \mathrm{I}_{4}(x=0.25,0.75)$. The lateral unit cell is a $(2 \times 2)$ unit cell with respect to the underlying octahedra (i.e., there are four metal halide octahedra per lateral unit cell) and the $(S-\mathrm{MBA})_{2} \mathrm{~Pb}_{0.25} \mathrm{Sn}_{0.75} \mathrm{I}_{4}$ structure serves as an example. (a) Top view of the inorganic layer along the $b$-axis. (b) Side view of the inorganic layer along the $a$ axis. The labels of bond angles are colored in black and bond lengths are colored in red. 
Table S5. Comparison of lattice parameters and unit cell volumes between input and relaxed structures of $(S-\mathrm{MBA})_{2} \mathrm{~Pb}_{1-x} \mathrm{Sn}_{x} \mathrm{I}_{4}(x=0,0.25,0.5,0.75,1)$. The input structures for $x=0$ and 1 were obtained from XRD. The input structures for $x=0.25$ and $x=0.5,0.75$ model alloy systems were artificially constructed by substituting a corresponding proportion of $\mathrm{Sn}(\mathrm{Pb})$ cations in the experimental structures of the pure $\mathrm{Pb}$ and pure $\mathrm{Sn}$ end point structures, i.e., $(S-\mathrm{MBA})_{2} \mathrm{PbI}_{4}$ and $(S \text {-MBA })_{2} \mathrm{SnI}_{4}$, respectively. The unit cells for $x=0.25$ and 0.75 were doubled within the $(a, c)$ plane forming a $(2 \times 2)$ arrangement. This makes it possible to accommodate the same 25:75 (75:25) stoichiometry in each inorganic layer.

\begin{tabular}{|c|c|c|c|c|c|c|c|c|}
\hline Compound & & $a(\AA)$ & $b(\AA)$ & $c(\AA)$ & $\alpha\left({ }^{\circ}\right)$ & $\beta\left(^{\circ}\right)$ & $\gamma\left({ }^{\circ}\right)$ & $V\left(\AA^{3}\right)$ \\
\hline \multirow{3}{*}{$\begin{array}{c}x=0, \\
\text { Pb100\% Sn0\% }\end{array}$} & Input (Exp.) & 8.90 & 28.86 & 9.31 & 90.0 & 90.0 & 90.0 & 2393 \\
\hline & $\mathrm{PBE}+\mathrm{TS}$ & 8.81 & 28.83 & 9.19 & 90.0 & 90.0 & 90.0 & 2333 \\
\hline & Deviation(\%) & -1.07 & -0.12 & -1.36 & & & & -2.54 \\
\hline$x=0.25$ & Input & 12.88 & 28.86 & 12.88 & 90.0 & 92.6 & 90.0 & 4787 \\
\hline \multirow{2}{*}{$\begin{array}{c}\text { Sn25\% Pb75\% } \\
\text { (with doubled unit cell) }\end{array}$} & $\mathrm{PBE}+\mathrm{TS}$ & 12.71 & 28.79 & 12.71 & 90.0 & 92.3 & 90.0 & 4651 \\
\hline & Deviation(\%) & -1.31 & -0.25 & -1.33 & & & & -2.84 \\
\hline \multirow{3}{*}{$\begin{array}{c}x=0.5 \\
\text { Sn50\% Pb50\% }\end{array}$} & Input & 8.91 & 28.73 & 9.36 & 90.0 & 90.0 & 90.0 & 2396 \\
\hline & $\mathrm{PBE}+\mathrm{TS}$ & 8.80 & 28.78 & 9.16 & 90.0 & 92.0 & 90.0 & 2320 \\
\hline & Deviation(\%) & -1.21 & 0.17 & -2.16 & & & & -3.18 \\
\hline \multirow{3}{*}{$\begin{array}{c}x=\mathbf{0 . 7 5} \\
\text { Sn75\% Pb25\% } \\
\text { (with doubled unit cell) }\end{array}$} & Input & 12.92 & 28.73 & 12.92 & 90.0 & 92.8 & 90.0 & 4792 \\
\hline & $\mathrm{PBE}+\mathrm{TS}$ & 12.72 & 28.75 & 12.72 & 90.0 & 92.2 & 90.0 & 4649 \\
\hline & Deviation(\%) & -1.53 & 0.07 & -1.59 & & & & -2.99 \\
\hline \multirow{3}{*}{$\begin{array}{c}x=1, \\
\text { Sn100\% Pb0\% }\end{array}$} & Input (Exp.) & 8.91 & 28.73 & 9.36 & 90.0 & 90.0 & 90.0 & 2396 \\
\hline & $\mathrm{PBE}+\mathrm{TS}$ & 8.84 & 28.72 & 9.18 & 90.0 & 90.0 & 90.0 & 2329 \\
\hline & Deviation(\%) & -0.84 & -0.05 & -1.92 & & & & -2.80 \\
\hline
\end{tabular}




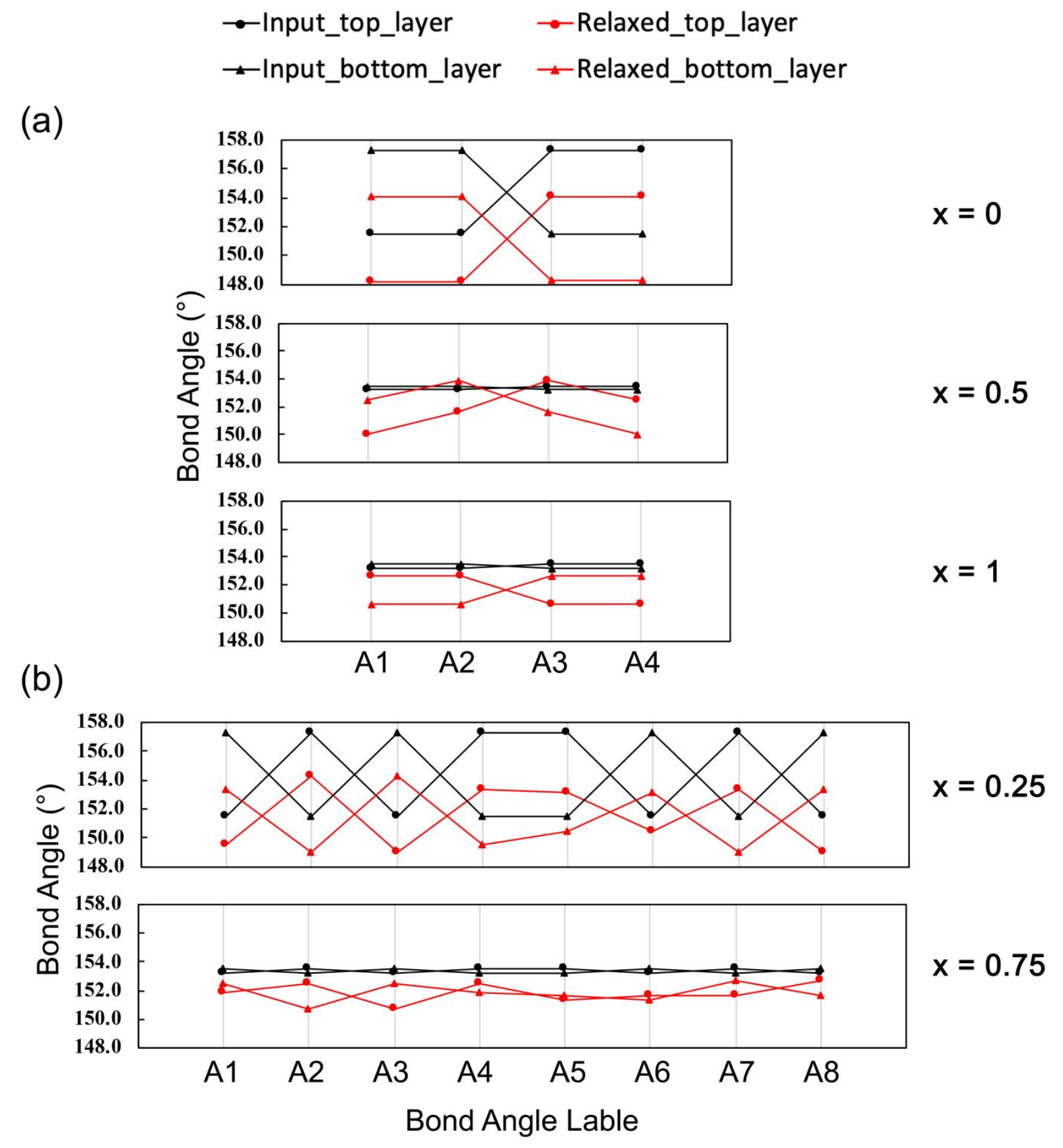

Figure S5. Comparison of bond angles between input (black data points) and DFT-PBE+TS relaxed (red data points) structures of (a) $(S-\mathrm{MBA})_{2} \mathrm{~Pb}_{1-x} \mathrm{Sn}_{x} \mathrm{I}_{4}(x=0,0.5,1)$ and (b) $(S-$ $\mathrm{MBA})_{2} \mathrm{~Pb}_{1-x} \mathrm{Sn}_{x} \mathrm{I}_{4}(x=0.25,0.75)$. The input structures for $x=0$ and 1 are experimental structures while the input structures for $x=0.25$ and $x=0.5,0.75$ model alloy systems are generated based on the experimental structures of $(S-\mathrm{MBA})_{2} \mathrm{PbI}_{4}$ and $(S-\mathrm{MBA})_{2} \mathrm{SnI}_{4}$, respectively. The label notation in (a) and (b) is the same as in Figure $\mathbf{S 3}$ and Figure $\mathbf{S 4}$. 
(a)

$\rightarrow$-Input_top_layer $\quad \rightarrow$ Relaxed_top_layer
$\rightarrow$ Input_bottom_layer $\rightarrow$ Relaxed_bottom_layer

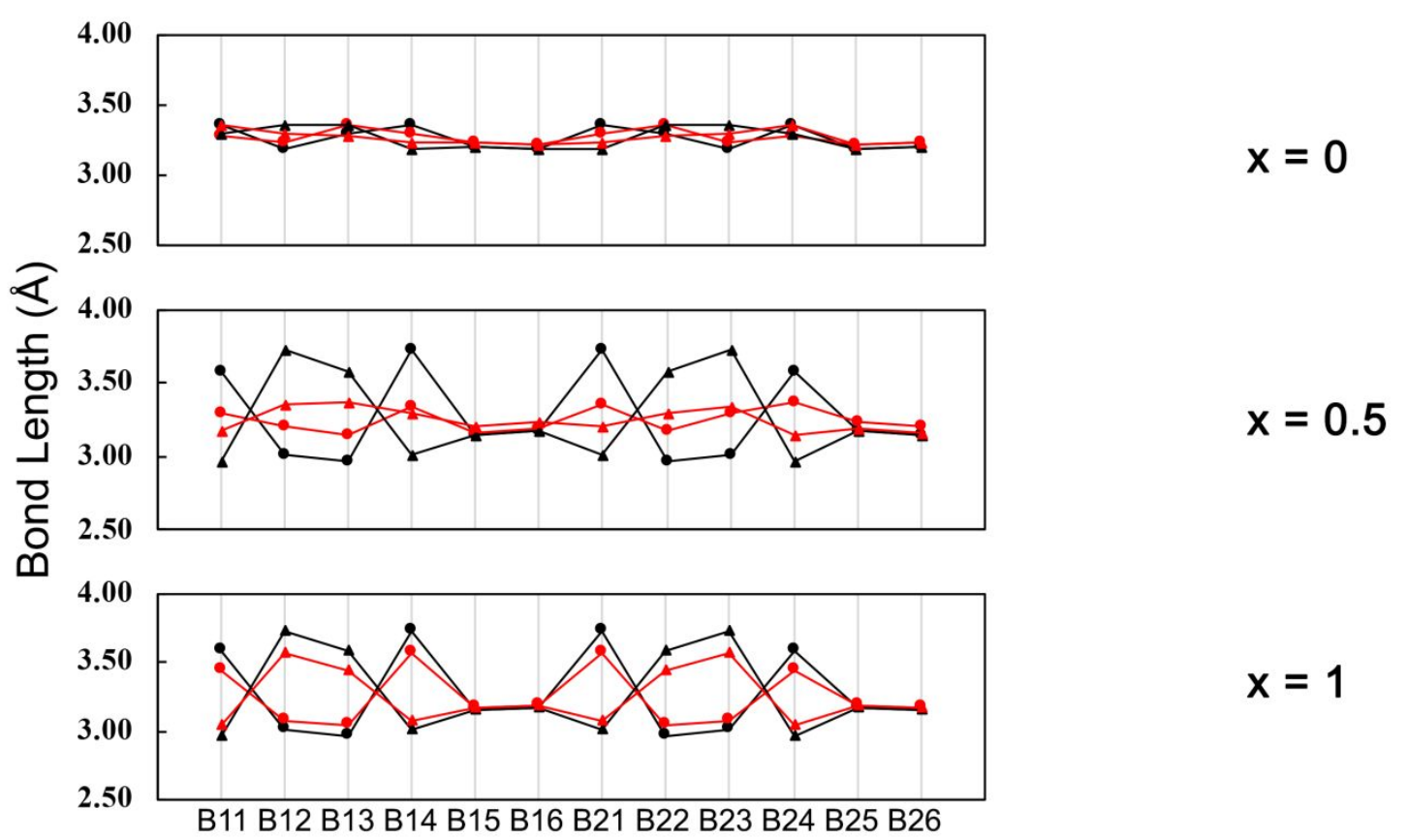

(b)

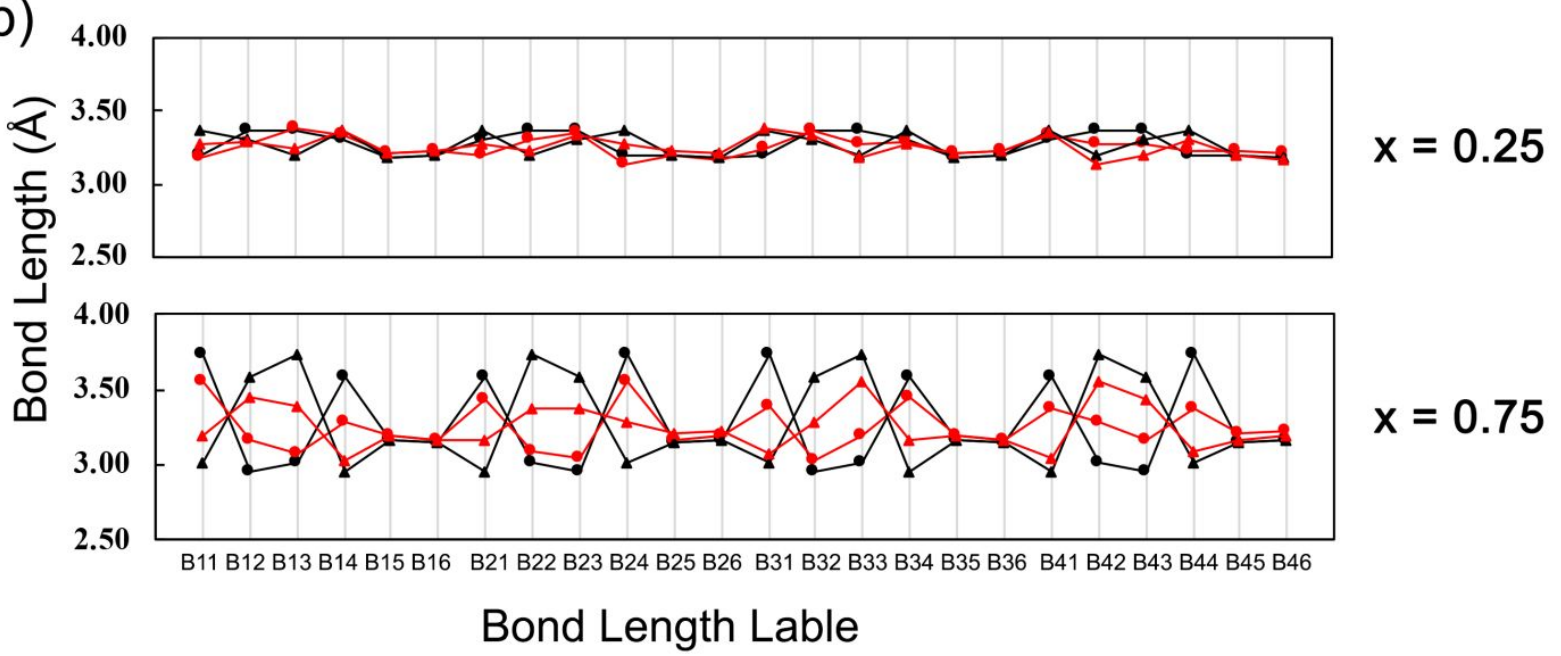

Figure S6. Comparison of bond lengths between input (black data points) and DFT-PBE+TS relaxed (red data points) structures of (a) $(S \text {-MBA) })_{2} \mathrm{~Pb}_{1-x} \mathrm{Sn}_{x} \mathrm{I}_{4}(x=0,0.5,1)$ and (b) $(S$ $\mathrm{MBA})_{2} \mathrm{~Pb}_{1-x} \mathrm{Sn}_{x} \mathrm{I}_{4}(x=0.25,0.75)$. The input structures for $x=0$ and 1 are experimental structures while the input structures for $x=0.25$ and $x=0.5,0.75$ model alloy systems are generated based on the experimental structures of $(S-\mathrm{MBA})_{2} \mathrm{PbI}_{4}$ and $(S-\mathrm{MBA})_{2} \mathrm{SnI}_{4}$, respectively. The label notation in (a) and (b) is the same as in Figure $\mathbf{S 3}$ and Figure $\mathbf{S 4}$. 


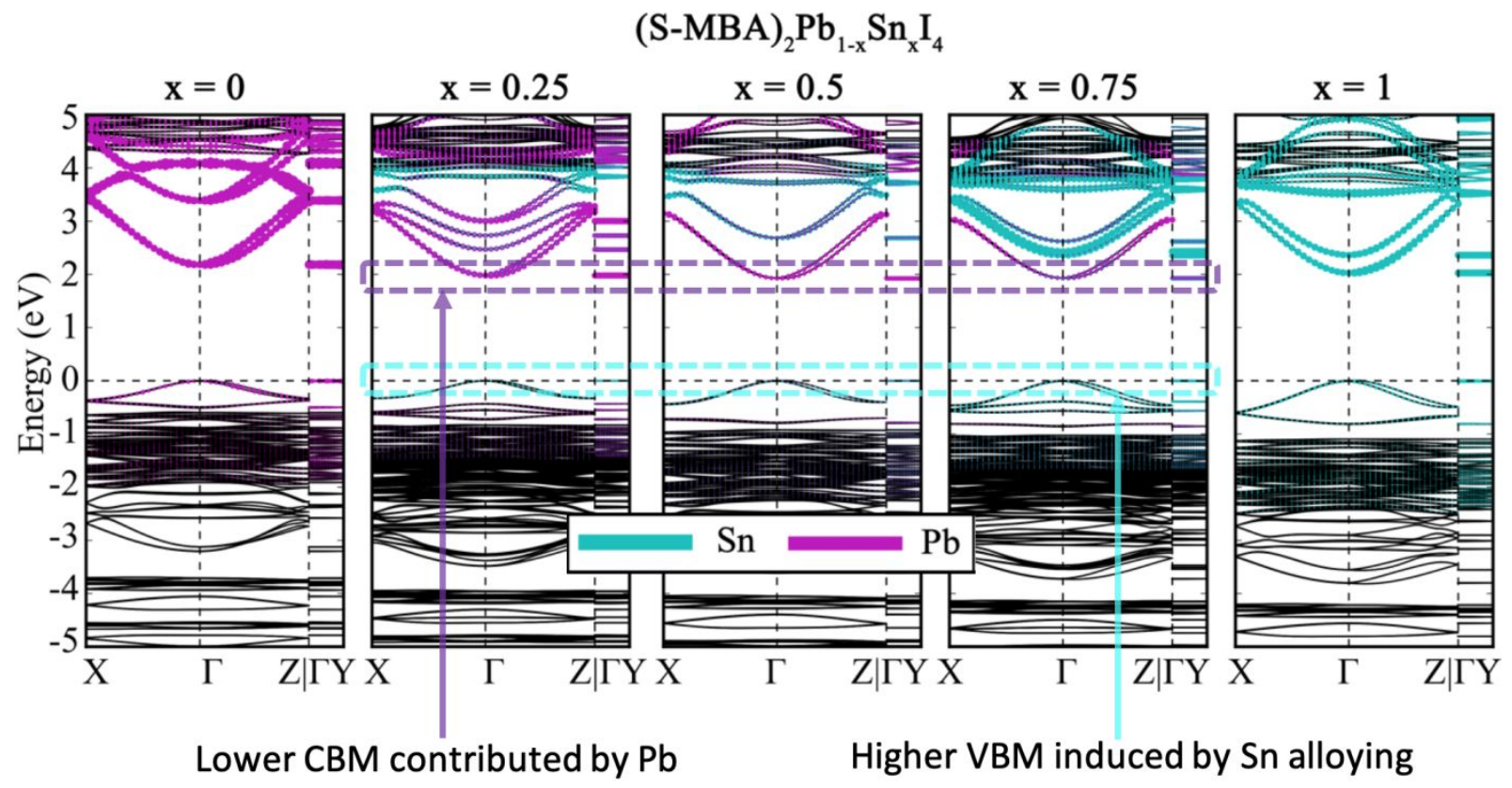

Figure S7. The computed DFT-HSE06+SOC electronic band structures of $(S-\mathrm{MBA})_{2} \mathrm{~Pb}_{1-x} \mathrm{Sn}_{x} \mathrm{I}_{4}(x$ $=0,0.25,0.5,0.75,1)$, highlighting the contributions of electronic states derived predominantly from $\mathrm{Sn}$ (cyan), and $\mathrm{Pb}$ (magenta). In particular, for the three computationally considered alloy systems $(x=0.25,0.5,0.75)$, the CBMs have a higher $\mathrm{Pb}$ contribution than the compositional average. The lowest unoccupied $\mathrm{Pb}$-derived states are lower in energy than the next lowest unoccupied states that have a higher Sn contribution. In contrast, the Sn-related VBM states are higher in energy than the highest-lying occupied states that have a predominant $\mathrm{Pb}$ cation contribution. Detailed plots of these contributions are shown in Figure S8 and Figure S9. The relative shifts between $\mathrm{Pb}$-derived states and $\mathrm{Sn}$-derived states accounts for the decrease of bandgaps of the alloyed systems, compared to non-alloyed ones $(x=0,1)$. 

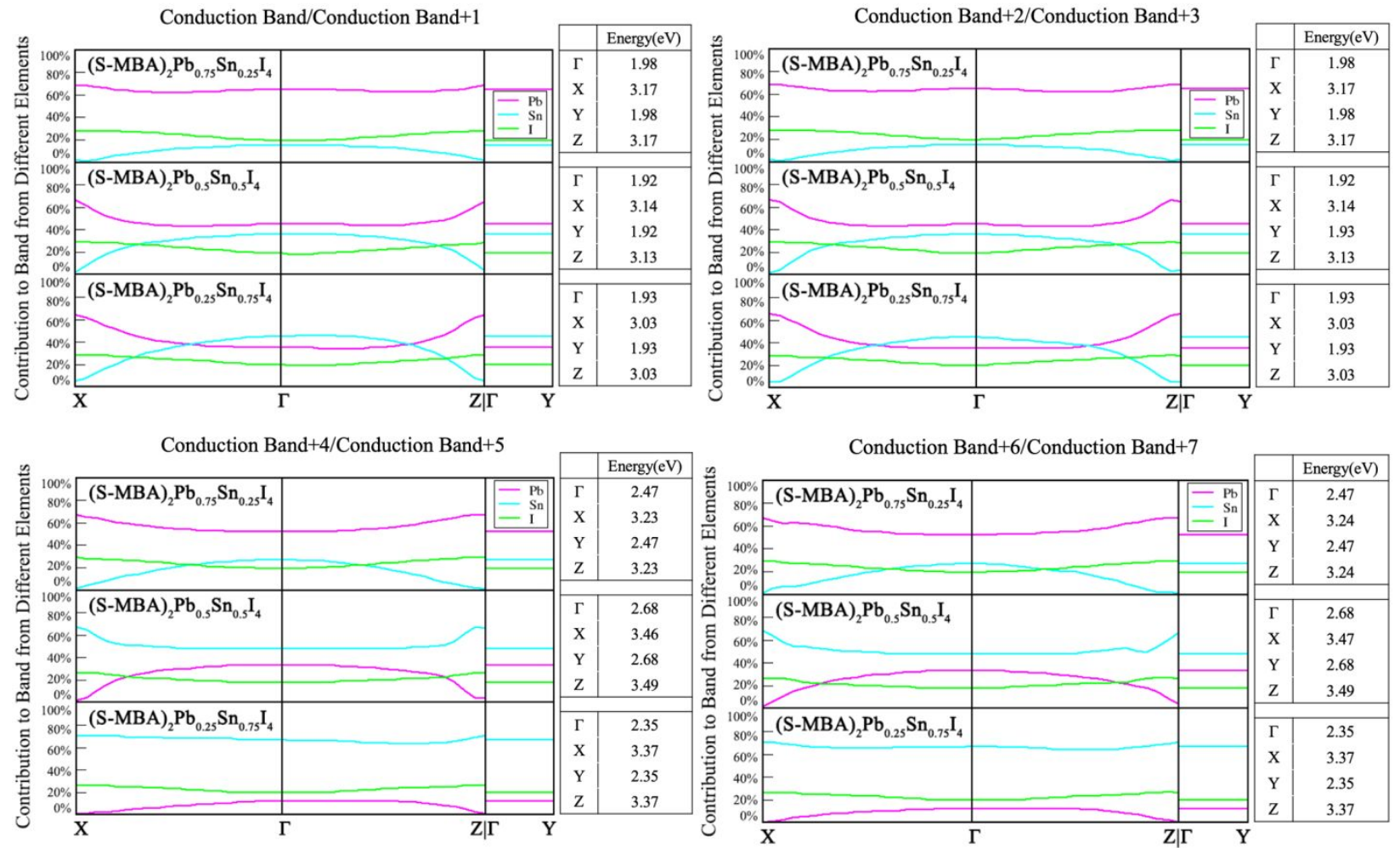

Figure S8. Mulliken decomposition of individual spin-orbit coupled bands for the model alloy system $(S-\mathrm{MBA})_{2} \mathrm{~Pb}_{1-x} \mathrm{Sn}_{x} \mathrm{I}_{4}(x=0.25,0.5$ and 0.75$)$ along the $\mathrm{k}$-space directions $\mathrm{X}-\Gamma, \Gamma-\mathrm{Z}$ and $\Gamma-$ $\mathrm{Y}$. Contributions from $\mathrm{Pb}$ (magenta), $\mathrm{Sn}$ (cyan) and I (green) are shown for the eight lowest-lying unoccupied spin-orbit coupled bands. For better comparison with Fig. $6 c$ in the main text, individual band energies at selected k-points are given in the accompanying tables. For the energetically lowest-lying conduction states, i.e., those near the $\Gamma$ associated with Conduction Band through Conduction $\mathrm{Band}+3$, the $\mathrm{Pb}: \mathrm{Sn}$ ratio is larger than the compositional $\mathrm{Pb}: \mathrm{Sn}$ ratio in the overall alloy in all cases. Note that each state has an essentially degenerate spin-orbit coupled counterpart and therefore only the Conduction Band, Conduction Band +2 , Conduction Band +4 and Conduction Band +6 are shown. 

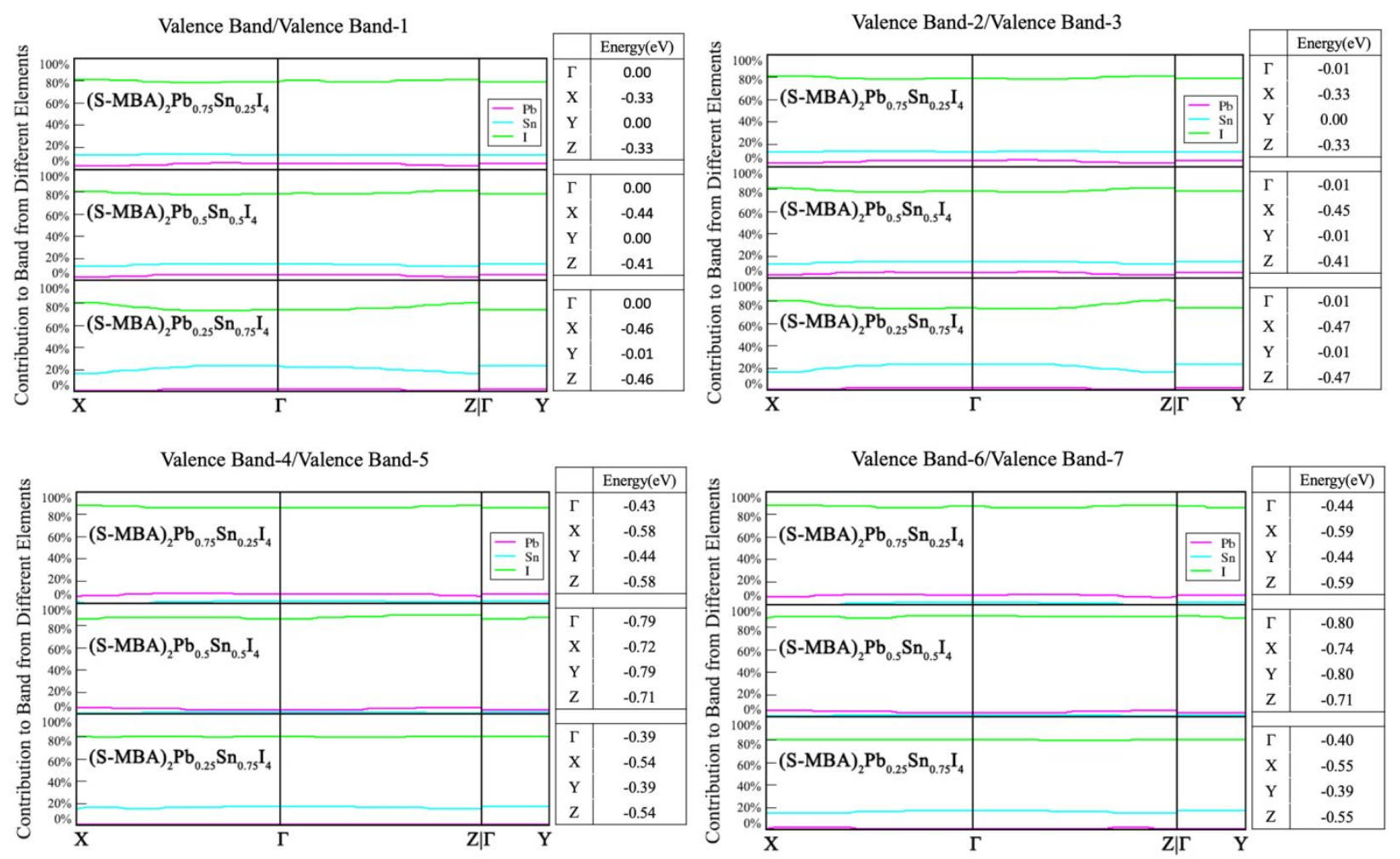

Figure S9. Mulliken decomposition of individual spin-orbit coupled bands for the model alloy system $(S-\mathrm{MBA})_{2} \mathrm{~Pb}_{1-x} \mathrm{Sn}_{x} \mathrm{I}_{4}(x=0.25,0.5$ and 0.75$)$ along the k-space directions $\mathrm{X}-\Gamma, \Gamma-\mathrm{Z}$ and $\Gamma-$ $\mathrm{Y}$. Contributions from $\mathrm{Pb}$ (magenta), $\mathrm{Sn}$ (cyan) and I (green) are shown for the eight highest-lying occupied spin-orbit coupled bands. For better comparison with Fig. $6 \mathrm{c}$ in the main text, individual band energies at selected k-points are given in the accompanying tables. For the energetically highest-lying valence states, i.e., those near the $\Gamma$ associated with Valence Band through Valence Band-3, the $\mathrm{Pb}: \mathrm{Sn}$ ratio is significantly lower than the compositional $\mathrm{Pb}: \mathrm{Sn}$ ratio in the overall alloy in all cases. Note that each state has an essentially degenerate spin-orbit coupled counterpart and therefore only the Valence Band, Valence Band-2, Valence Band-4 and Valence Band-6 are shown. 


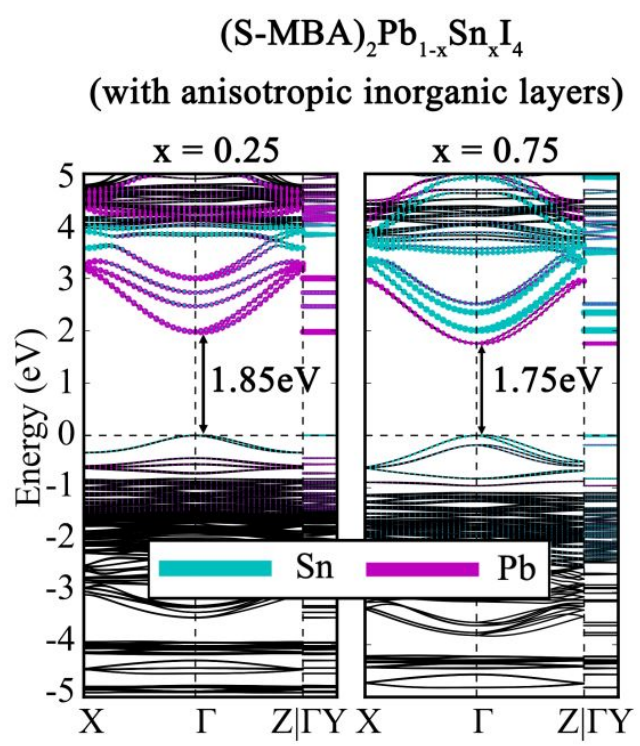

Figure S10. The computed DFT-HSE06+SOC electronic band structures of $(S-\mathrm{MBA})_{2} \mathrm{~Pb}_{1-x} \mathrm{Sn}_{x} \mathrm{I}_{4}$ $(x=0.25,0.75)$ based on $\mathrm{c}(2 \times 2)$ lateral unit cells (see Figure S3). In this case, there are only two $\mathrm{Sn} / \mathrm{Pb}$ cation sites in each inorganic plane and the overall stoichiometry can only be accomplished by including planes of different composition. In the examples shown, pure $\mathrm{Pb}(\mathrm{Sn})$ and mixed $\mathrm{Pb} / \mathrm{Sn}$ inorganic planes alternate. The predicted fundamental band gaps are reduced by more than $0.1 \mathrm{eV}$ compared to their counterparts with isotropic inorganic layers.

A simple computational experiment reveals that the predicted band gaps can indeed vary significantly as a consequence of local inhomogeneities. In Figure S10, uneven cation distributions in the inorganic planes are probed for the particular case of alternating pure $\mathrm{Pb}(\mathrm{Sn})$ and 50-50 mixed $\mathrm{Pb} / \mathrm{Sn}$ planes at compositions $x=0.25(0.75)$. The result is a significant drop of the overall band gaps, by more than $0.1 \mathrm{eV}$ in both cases. The actual inhomogeneities encountered in real samples are almost certainly different from the simple cases explored here (the determination of the detailed elemental distributions expected in the alloyed samples lies beyond the scope of this work). Importantly, differences in elemental distribution can entirely account for most of the remaining observed differences between the optical gaps of the real materials and the fundamental gaps computed for the alloyed structure models. 
VIII. AFM images and magnetic CP-AFM data
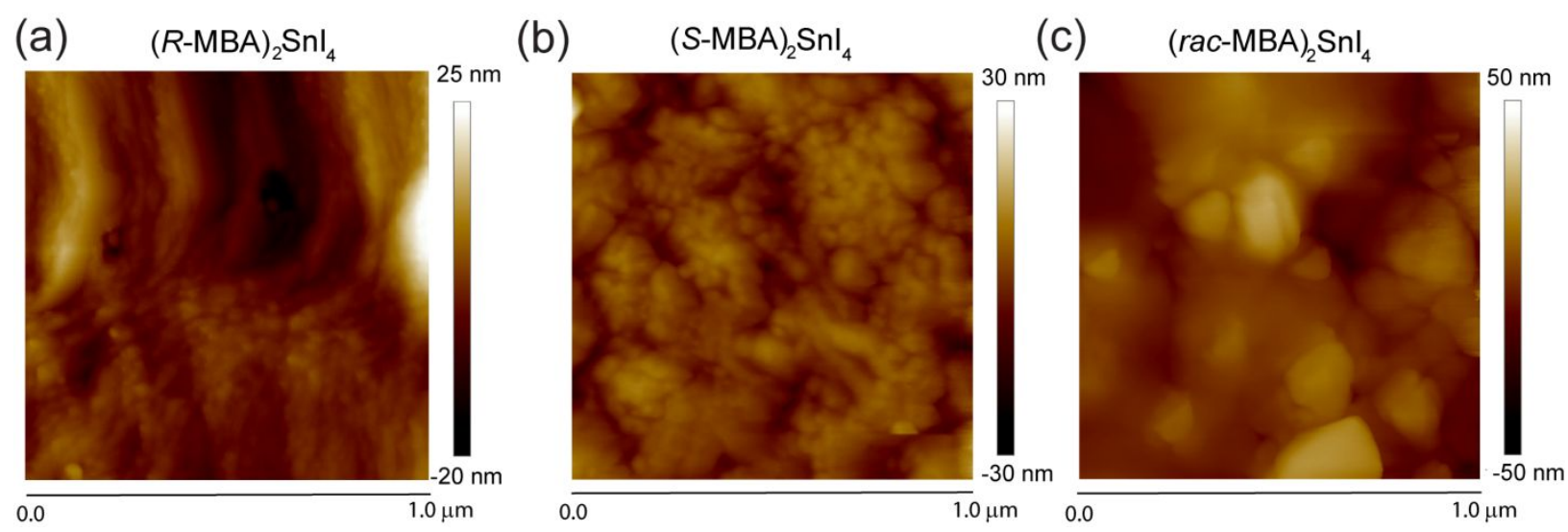

Figure S11. AFM images of $(R-\mathrm{MBA})_{2} \mathrm{SnI}_{4},(S-\mathrm{MBA})_{2} \mathrm{SnI}_{4}$, and $(\mathrm{rac}-\mathrm{MBA})_{2} \mathrm{SnI}_{4}$ thin films. 

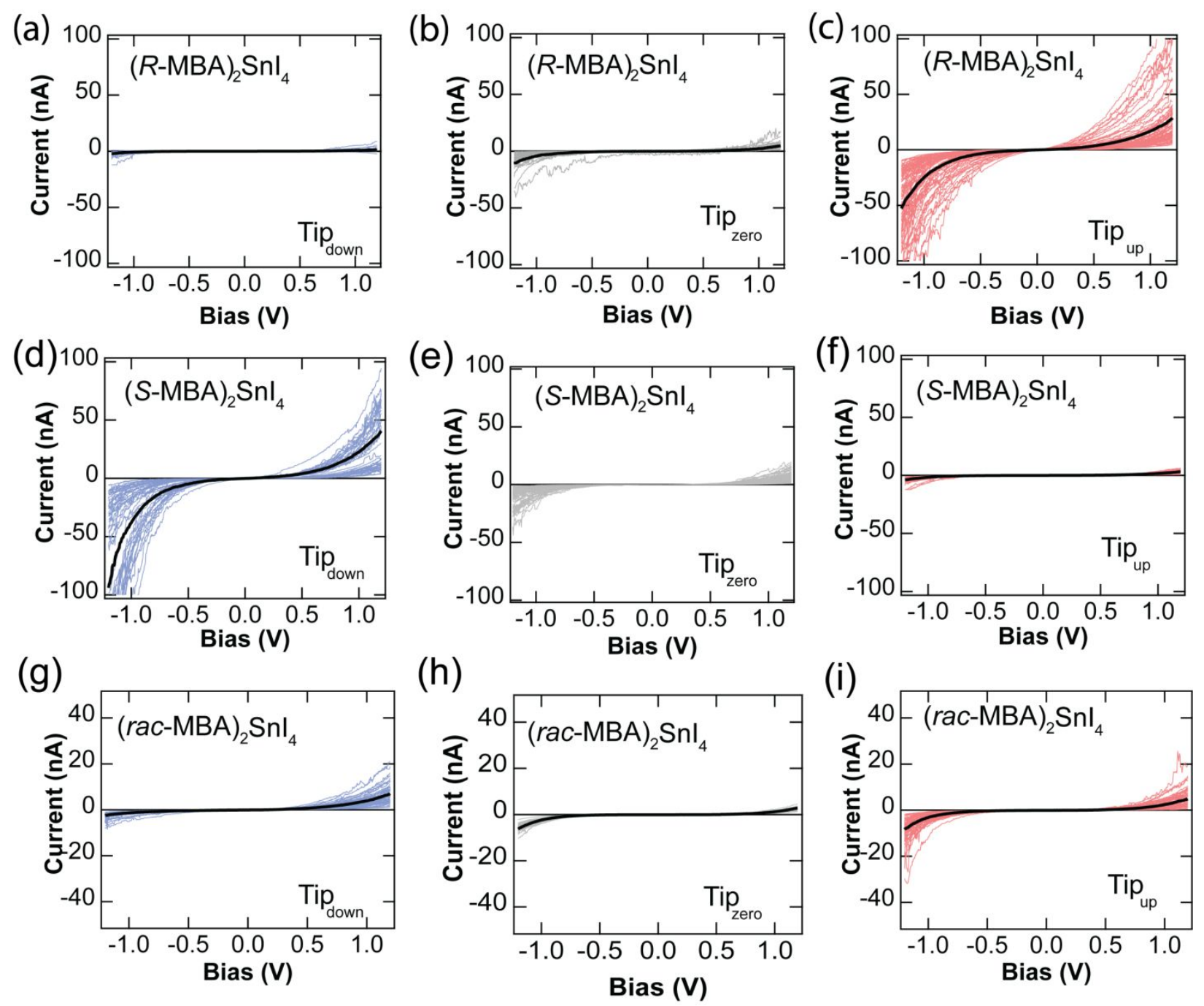

Figure S12. Raw and averaged current-voltage $(I-V)$ curves from the mCP-AFM measurements of chiral perovskite thin films $(\sim 50 \mathrm{~nm})(R-\mathrm{MBA})_{2} \mathrm{SnI}_{4}(\mathbf{a}-\mathbf{c}),(S-\mathrm{MBA})_{2} \mathrm{SnI}_{4}(\mathbf{b}-\mathbf{f})$, and $(\mathrm{rac}-$ MBA) $)_{2} \mathrm{SnI}_{4}$ (g-i). The statistic $95 \%$ confident limit is defined as $\left(\bar{x}-z^{*} \times \frac{\sigma}{\sqrt{n}}, \bar{x}+z^{*} \times \frac{\sigma}{\sqrt{n}}\right)$, where $\bar{x}, z^{*}, \sigma$ and $n$ are the mean value, 1.96 (for $95 \%$ confidence), standard deviation, and the total scan number, respectively. 


\section{Geometry information for electronic structure calculations}

This section provides the geometric information for electronic structure calculations. Unit cell parameters and atomic coordinates (Cartesian) are listed in FHI-aims' geometry format. Unit cell type and relaxation strategy are next to the name of each system. All numbers related to structural information are given in A. FHI-aims' geometry format can be read by standard viewing programs (e. g., jmol) and can be converted into other formats using Vesta.

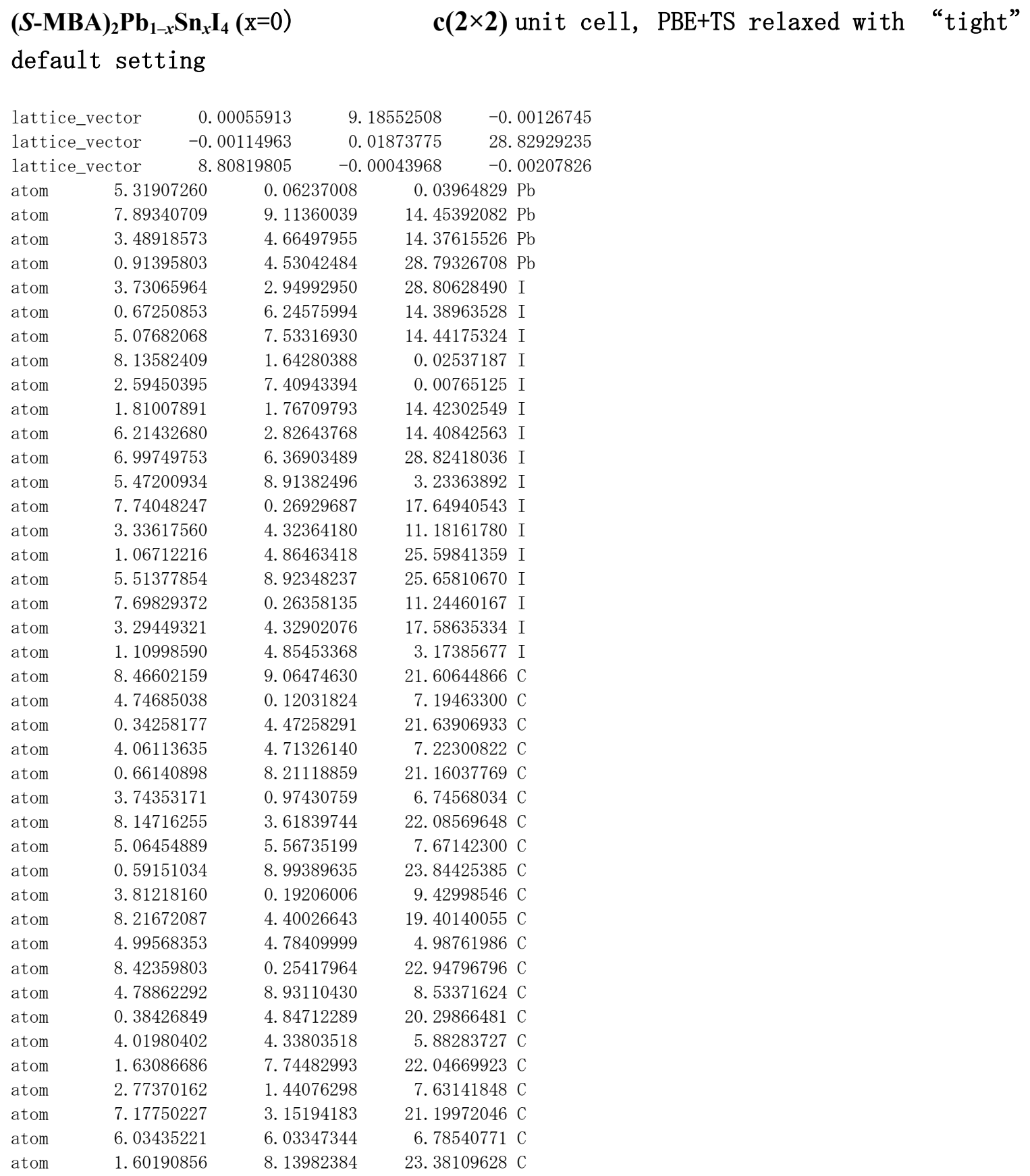




\begin{tabular}{|c|c|c|c|}
\hline atom & 2. 80199728 & 1. 04595732 & 8. 96596472 \\
\hline atom & 7. 20626997 & 3. 54654485 & 19.86514543 \\
\hline atom & 6. 00593358 & 5. 63818558 & 5. 45106924 \\
\hline atom & 5. 41531845 & 5. 33610722 & 23. 69531024 \\
\hline atom & 7. 79663332 & 3. 85007573 & 9. 27945905 \\
\hline atom & 3. 39264687 & 0.74302860 & 19.55113705 \\
\hline atom & 1. 01161877 & 8. 44243847 & 5. 13721388 \\
\hline atom & 5. 86299163 & 3. 52133634 & 25. 43231280 \\
\hline atom & 7. 34832830 & 5. 66458250 & 11.01662555 \\
\hline atom & 2. 94512009 & 8. 11330090 & 17. 81369899 \\
\hline atom & 1. 45933441 & 1. 07076637 & 3. 40043302 \\
\hline atom & 5. 70048152 & 5. 00631746 & 25. 14479322 \\
\hline atom & 7. 51149709 & 4. 17977886 & 10. 72885930 \\
\hline atom & 3. 10751623 & 0.41279145 & 18. 10184492 \\
\hline atom & 1. 29680911 & 8. 77156800 & 3. 68759768 \\
\hline atom & 6. 30138438 & 6. 15804737 & 22. 98873521 \\
\hline atom & 6. 91073071 & 3. 02794629 & 8. 57296462 \\
\hline atom & 2. 50647400 & 1. 56505472 & 20.25737931 \\
\hline atom & 1. 89733857 & 7. 62032783 & 5. 84405647 \\
\hline atom & 4. 32731085 & 4. 77075034 & 23.01733407 \\
\hline atom & 0.07651005 & 4. 41582505 & 8. 60363566 \\
\hline atom & 4. 48070687 & 0.17796927 & 20. 22932422 \\
\hline atom & 8. 73193511 & 9. 00788913 & 5. 81283511 \\
\hline atom & 6. 10637280 & 6. 40679376 & 21. 63193821 \\
\hline atom & 7. 10601463 & 2. 77895779 & 7. 21628799 \\
\hline atom & 2. 70134767 & 1. 81408031 & 21. 61411463 \\
\hline atom & 1. 70199889 & 7. 37187358 & 7. 20084699 \\
\hline atom & 4. 13633106 & 5. 01736143 & 21. 65916715 \\
\hline atom & 0.26777409 & 4. 16894832 & 7. 24559849 \\
\hline atom & 4. 67153898 & 0.42484224 & 21.58743042 \\
\hline atom & 8. 54054549 & 8. 76144334 & 7. 17096184 \\
\hline atom & 5. 02367946 & 5. 83887065 & 20. 96613911 \\
\hline atom & 8. 18881815 & 3. 34679965 & 6. 55056730 \\
\hline atom & 3. 78401301 & 1. 24634342 & 22.28016129 \\
\hline atom & 0.61926935 & 7. 94011783 & 7. 86631331 \\
\hline atom & 0.55983906 & 0.29641337 & 25. 27559328 \\
\hline atom & 3. 84383755 & 8. 89056018 & 10. 85907369 \\
\hline atom & 8. 24783685 & 4. 88760335 & 17. 97107734 \\
\hline atom & 4. 96466063 & 4. 29625366 & 3. 55755754 \\
\hline atom & 1. 86754709 & 0.88565315 & 25. 77004636 \\
\hline atom & 2. 53612835 & 8. 30254777 & 11. 35497538 \\
\hline atom & 6. 94018336 & 5. 47648398 & 17. 47597973 \\
\hline atom & 6. 27232292 & 3. 70742973 & 3. 06255367 \\
\hline atom & 7. 73298008 & 0.27063880 & 20.90326377 \\
\hline atom & 5. 48017391 & 8. 91423371 & 6. 48943918 \\
\hline atom & 1. 07522061 & 4. 86412154 & 22.34322428 \\
\hline atom & 3. 32840070 & 4. 32198148 & 7. 92721975 \\
\hline atom & 0.68896782 & 7. 93394747 & 20. 10781472 \\
\hline atom & 3. 71644884 & 1. 25150613 & 5. 69307920 \\
\hline atom & 8. 11971634 & 3. 34141481 & 23. 13835526 \\
\hline atom & 5. 09169345 & 5. 84487534 & 8. 72391307 \\
\hline atom & 7. 63043187 & 0.90754953 & 23. 30954467 \\
\hline atom & 5. 58205803 & 8. 27804769 & 8. 89537372 \\
\hline atom & 1. 17763070 & 5. 50016691 & 19. 93684440 \\
\hline atom & 3. 22652391 & 3. 68481640 & 5.52122011 \\
\hline atom & 2. 44096063 & 7. 10709636 & 21. 69745599 \\
\hline atom & 1. 96380482 & 2. 07841751 & 7. 28149929 \\
\hline atom & 6. 36741298 & 2. 51441265 & 21.54940259 \\
\hline atom & 6. 84432112 & 6. 67122456 & 7. 13492317 \\
\hline atom & 2. 39427924 & 7. 80058559 & 24. 04929612 \\
\hline atom & 2. 00919836 & 1. 38521999 & 9. 63361368 \\
\hline atom & 6. 41367500 & 3. 20727604 & 19. 19724965 \\
\hline
\end{tabular}




\begin{tabular}{|c|c|c|c|}
\hline atom & 6. 79864146 & 5. 97720353 & 4. $78316649 \mathrm{H}$ \\
\hline atom & 3. 65808414 & 5. 24922782 & 25. $81630958 \mathrm{H}$ \\
\hline atom & 0.74606455 & 3. 93826933 & 11. $40196267 \mathrm{H}$ \\
\hline atom & 5. 15002266 & 0.65442958 & 17. 42993780 \\
\hline atom & 8. 06224059 & 8. 52817006 & 3. 01511161 \\
\hline atom & 4. 64524484 & 6. 62279398 & 25. 98055979 \\
\hline atom & 8.56776368 & 2. 56376738 & $11.56428252 \mathrm{H}$ \\
\hline atom & 4. 16361266 & 2. 02860949 & 17. $26596929 \mathrm{H}$ \\
\hline atom & 0.24118375 & 7. 15513067 & 2. 85209950 \\
\hline atom & 4. 80848763 & 5. 33098745 & 27. 03350928 \\
\hline atom & 8. 40396684 & 3. 85505253 & 12. 61737290 \\
\hline atom & 3. 99909249 & 0.73732590 & 16. $21299609 \mathrm{H}$ \\
\hline atom & 0.40359948 & 8. 44718502 & 1. $79927961 \mathrm{H}$ \\
\hline atom & 6. 19331465 & 3. 34690640 & 26. 46430708 \\
\hline atom & 7. 01830349 & 5. 83852228 & 12. 04877580 \\
\hline atom & 2. 61486946 & 7. 93859619 & 16. $78174665 \mathrm{H}$ \\
\hline atom & 1. 78947093 & 1. 24432232 & 2. $36823742 \mathrm{H}$ \\
\hline atom & 6. 62444593 & 3. 10866595 & 24. $76272101 \mathrm{H}$ \\
\hline atom & 6. 58645521 & 6. 07704571 & 10. 34733920 \\
\hline atom & 2. 18350971 & 7. 70115020 & 18. 48346882 \\
\hline atom & 2. 22115048 & 1. 48356918 & 4. $06953472 \mathrm{H}$ \\
\hline atom & 4. 93305411 & 2. 96218102 & $25.27106154 \mathrm{H}$ \\
\hline atom & 8. 27795468 & 6. 22421913 & $10.85522762 \mathrm{H}$ \\
\hline atom & 3. 87489901 & 7. 55396641 & 17. $97529127 \mathrm{H}$ \\
\hline atom & 0.52968438 & 1. 63046012 & 3. 56146810 \\
\hline atom & 6. 61554671 & 5. 53303205 & 25. $44941172 \mathrm{H}$ \\
\hline atom & 6. 59681867 & 3. 65252284 & 11. $03371738 \mathrm{H}$ \\
\hline atom & 2. 19267070 & 0.93971527 & 17. 79688219 \\
\hline atom & 2. 21156967 & 8. 24429132 & 3. $38307065 \mathrm{H}$ \\
\hline atom & 7. 16963271 & 6. 58252786 & 23. $49372562 \mathrm{H}$ \\
\hline atom & 6. 04242008 & 2. 60349828 & 9. $07790257 \mathrm{H}$ \\
\hline atom & 1. 63825483 & 1. 98936830 & 19. $75215593 \mathrm{H}$ \\
\hline atom & 2. 76556435 & 7. 19550286 & 5. $33933092 \mathrm{H}$ \\
\hline atom & 3. 62823968 & 4. 11949104 & 23.54325510 \\
\hline atom & 0.77550128 & 5. 06714259 & 9. $12953283 \mathrm{H}$ \\
\hline atom & 5. 18049732 & 8. 71235562 & 19. $70229807 \mathrm{H}$ \\
\hline atom & 8. 03290371 & 0.47410139 & 5. $28794602 \mathrm{H}$ \\
\hline atom & 6. 82773577 & 7. 01712616 & $21.09262486 \mathrm{H}$ \\
\hline atom & 6. 38477823 & 2. 16851206 & 6. $67697788 \mathrm{H}$ \\
\hline atom & 1. 97988071 & 2. 42447831 & $22.15314955 \mathrm{H}$ \\
\hline atom & 2. 42309790 & 6. 76152643 & 7. $74051589 \mathrm{H}$ \\
\hline atom & 3. 31719724 & 4. 53694897 & 21. 12748250 \\
\hline atom & 1. 08698921 & 4. 64928637 & 6. $71395224 \mathrm{H}$ \\
\hline atom & 5. 49129579 & 9. 13014897 & 22. $11799821 \mathrm{H}$ \\
\hline atom & 7. 72097047 & 0.05665552 & 7. $70373998 \mathrm{H}$ \\
\hline atom & 4. 87707692 & 6. 00346594 & 19. $89960455 \mathrm{H}$ \\
\hline atom & 8. 33569150 & 3. 18200835 & 5. 48412475 \\
\hline atom & 3. 93050525 & 1. 41102481 & 23. 34667914 \\
\hline atom & 0.47236315 & 7. 77557672 & 8. $93280894 \mathrm{H}$ \\
\hline atom & 8. 57229159 & 1. 04743269 & 25. $37631303 \mathrm{H}$ \\
\hline atom & 4. 63919816 & 8. 13872542 & 10. 96235152 \\
\hline atom & 0.23533050 & 5. 63952736 & 17. 86994779 \\
\hline atom & 4. 16903144 & 3. 54468635 & 3. 45462032 \\
\hline atom & 0.57014055 & 7. 43871610 & 25. $97882228 \mathrm{H}$ \\
\hline atom & 3. 83279017 & 1. 74814126 & $11.56517415 \mathrm{H}$ \\
\hline atom & 8. 23697032 & 2. 84448824 & 17. 26634500 \\
\hline atom & 4. 97465451 & 6. 33945098 & 2. 85287448 \\
\hline atom & 7. 88767584 & 8. 23218651 & 26. $08538885 \mathrm{H}$ \\
\hline atom & 5. 32478596 & 0.95601293 & 11. $66971446 \mathrm{H}$ \\
\hline atom & 0.92041095 & 3. 63741436 & 17. 16194304 \\
\hline atom & 3. 48349604 & 5. 54624481 & 2. 74587987 \\
\hline atom & 0.29919538 & 8. 58514948 & 27. 17859911 \\
\hline
\end{tabular}




$\begin{array}{lllrl}\text { atom } & 4.10897400 & 0.60119978 & 12.76396107 \mathrm{H} \\ \text { atom } & 8.51112952 & 3.99135273 & 16.06716508 \mathrm{H} \\ \text { atom } & 4.70169617 & 5.19295743 & 1.65346513 \mathrm{H} \\ \text { atom } & 2.69605275 & 0.17007806 & 25.72791172 \mathrm{H} \\ \text { atom } & 1.70794602 & 9.01847942 & 11.31270145 \mathrm{H} \\ \text { atom } & 6.11168716 & 4.76090773 & 17.51827870 \mathrm{H} \\ \text { atom } & 7.10073490 & 4.42310377 & 3.10468445 \mathrm{H} \\ \text { atom } & 1.77417124 & 1.24805437 & 26.80080535 \mathrm{H} \\ \text { atom } & 2.63014592 & 7.94136639 & 12.38612662 \mathrm{H} \\ \text { atom } & 7.03382870 & 5.83820158 & 16.44498013 \mathrm{H} \\ \text { atom } & 6.17860212 & 3.34546093 & 2.03167592 \mathrm{H} \\ \text { atom } & 2.12603012 & 1.74552239 & 25.14145072 \mathrm{H} \\ \text { atom } & 2.27668123 & 7.44201025 & 10.72777124 \mathrm{H} \\ \text { atom } & 6.68144217 & 6.33677166 & 18.10381583 \mathrm{H} \\ \text { atom } & 6.53113995 & 2.84730578 & 3.69066695 \mathrm{H} \\ \text { atom } & 4.62284930 & 5.57650841 & 26.04641414 \mathrm{~N} \\ \text { atom } & 8.58970243 & 3.61004807 & 11.63021058 \mathrm{~N} \\ \text { atom } & 4.18539917 & 0.98232586 & 17.20006528 \mathrm{~N} \\ \text { atom } & 0.21870477 & 8.20142333 & 2.78643485 \mathrm{~N} \\ \text { atom } & 0.11480264 & 8.35344803 & 26.18796542 \mathrm{~N} \\ \text { atom } & 4.28985898 & 0.83400967 & 11.77288115 \mathrm{~N} \\ \text { atom } & 8.69352082 & 3.75875747 & 17.05802123 \mathrm{~N} \\ \text { atom } & 4.51865052 & 5.42494699 & 2.64427730 \mathrm{~N}\end{array}$

\section{$(S-M B A){ }_{2} \mathbf{P b}_{1-x} \mathbf{S n}_{x} \mathbf{I}_{4}(\mathrm{x}=0.25) \quad(2 \times 2)$ unit cell, PBE+TS relaxed with "tight" default setting}

$\begin{array}{lcccc}\text { lattice_vector } & 12.71516642 & 0.00001647 & 0.03267429 \\ \text { lattice_vector } & 0.00011691 & 28.79412258 & 0.00037232 \\ \text { lattice_vector } & -0.53815764 & -0.00007709 & 12.70154578 \\ \text { atom } & 3.72795713 & 28.75345196 & 3.89152917 \mathrm{Sn} \\ \text { atom } & 11.49335295 & 14.35684981 & 12.02668160 \mathrm{Sn} \\ \text { atom } & 9.71719385 & 28.75442755 & 10.26231694 \mathrm{~Pb} \\ \text { atom } & 5.50405474 & 14.35888757 & 5.65552803 \mathrm{~Pb} \\ \text { atom } & 11.78179854 & 14.43486404 & 5.73914273 \mathrm{~Pb} \\ \text { atom } & 5.07671425 & 14.43201600 & 12.08228921 \mathrm{~Pb} \\ \text { atom } & 10.14447242 & 0.03559736 & 3.83531712 \mathrm{~Pb} \\ \text { atom } & 3.43946203 & 0.03768070 & 10.17845714 \mathrm{~Pb} \\ \text { atom } & 0.47425451 & 0.02527658 & 4.70341655 \mathrm{I} \\ \text { atom } & 6.54394975 & 0.03043673 & 11.08940221 \mathrm{I} \\ \text { atom } & 8.67727792 & 14.42751470 & 4.82831082 \mathrm{I} \\ \text { atom } & 2.03189375 & 14.42220362 & 11.18172731 \mathrm{I} \\ \text { atom } & 10.80614709 & 14.36576913 & 8.96594171 \mathrm{I} \\ \text { atom } & 4.69018997 & 14.36780087 & 2.52985048 \mathrm{I} \\ \text { atom } & 4.41536910 & 28.76274180 & 6.95198514 \mathrm{I} \\ \text { atom } & 11.06930472 & 28.76484339 & 0.68667732 \mathrm{I} \\ \text { atom } & 9.15918517 & 28.78684514 & 7.02683909 \mathrm{I} \\ \text { atom } & 3.09358571 & 28.79116980 & 0.64459984 \mathrm{I} \\ \text { atom } & 12.66580744 & 14.39429502 & 2.57200804 \mathrm{I} \\ \text { atom } & 6.06206786 & 14.39000169 & 8.89157918 \mathrm{I} \\ \text { atom } & 2.25689472 & 14.40185358 & 6.44090968 \mathrm{I} \\ \text { atom } & 8.38500135 & 14.39531235 & 12.78509593 \mathrm{I} \\ \text { atom } & 0.24875586 & 0.00494077 & 9.44416408 \mathrm{I} \\ \text { atom } & 6.83614051 & -0.00171049 & 3.13263323 \mathrm{I} \\ \text { atom } & 10.06111923 & 25.56493552 & 10.13203796 \mathrm{I} \\ \text { atom } & 3.96781966 & 25.60196223 & 3.76497181 \mathrm{I} \\ \text { atom } & 5.16015794 & 11.16803811 & 5.78593735 \mathrm{I} \\ \text { atom } & 11.25343826 & 11.20460907 & 12.15305382 \mathrm{I} \\ \text { atom } & 11.90860226 & 17.62934619 & 5.43655404 \mathrm{I} \\ \text { atom } & 5.27324770 & 17.62380362 & 11.77223499 \mathrm{I} \\ \text { atom } & 9.94813936 & 3.22746697 & 4.14548802 \mathrm{I} \\ \text { atom } & 3.31272667 & 3.23244084 & 10.48104141 \mathrm{I} \\ \text { atom } & 10.08702545 & 3.17026157 & 10.15440470 \mathrm{I} \\ \text { atom } & 3.99904902 & 3.13413115 & 3.79405308 \mathrm{I}\end{array}$




\begin{tabular}{|c|c|c|c|}
\hline atom & 5.13426267 & 17.56780570 & $5.76343641 \mathrm{I}$ \\
\hline atom & 11.22232783 & 17.53106707 & $12.12366968 \mathrm{I}$ \\
\hline tom & 11.88172643 & 11.22223413 & 5.40634483 I \\
\hline tom & 5.24965506 & 11.22380648 & $11.73787273 \mathrm{I}$ \\
\hline tom & 9.97154876 & 25.62118604 & $4.18006709 \mathrm{I}$ \\
\hline tom & 3.33952485 & 25.61914194 & $10.51165369 \mathrm{I}$ \\
\hline atom & 12.02999901 & 7.22227447 & $12.38888870 \mathrm{C}$ \\
\hline atom & 5.94712306 & 7.21389321 & $6.02389720 \mathrm{C}$ \\
\hline tom & 3.19124775 & 21.61930186 & $3.52889694 \mathrm{C}$ \\
\hline atom & 9.27424464 & 21.61091597 & $9.89386698 \mathrm{C}$ \\
\hline atom & 9.73347840 & 7.18217150 & $3.35397402 \mathrm{C}$ \\
\hline atom & 3.11345069 & 7.18085298 & $9.68568638 \mathrm{C}$ \\
\hline atom & 12.10786234 & 21.57791682 & $6.23185041 \mathrm{C}$ \\
\hline atom & 5.48792831 & 21.57915815 & $12.56392426 \mathrm{C}$ \\
\hline atom & 7.25854685 & 7.65820619 & $6.16352863 \mathrm{C}$ \\
\hline atom & 0.62590130 & 7.66879167 & $12.49155915 \mathrm{C}$ \\
\hline atom & 1.88019377 & 22.06581265 & $3.39344324 \mathrm{C}$ \\
\hline atom & 7.96281302 & 22.05519422 & $9.75430452 \mathrm{C}$ \\
\hline atom & 3.03316828 & 6.73921000 & $8.36841673 \mathrm{C}$ \\
\hline atom & 9.65610170 & 6.73950666 & $2.03687898 \mathrm{C}$ \\
\hline atom & 12.18806747 & 21.13617608 & $7.54907861 \mathrm{C}$ \\
\hline atom & 6.10346236 & 21.13654668 & $1.17946897 \mathrm{C}$ \\
\hline atom & 6.63525925 & 4.97659365 & $6.65261567 \mathrm{C}$ \\
\hline atom & 0.54647927 & 4.98635551 & $0.28310597 \mathrm{C}$ \\
\hline atom & 2.49778912 & 19.38333205 & $2.90034435 \mathrm{C}$ \\
\hline atom & 8.58608389 & 19.37362498 & $9.26511914 \mathrm{C}$ \\
\hline atom & 2.50928583 & 9.41803917 & $8.97544917 \mathrm{C}$ \\
\hline atom & 9.13480263 & 9.41969090 & $2.63989543 \mathrm{C}$ \\
\hline atom & -0.00314274 & 23.81502497 & $6.90965594 \mathrm{C}$ \\
\hline atom & 6.62466253 & 23.81677272 & $0.57648795 \mathrm{C}$ \\
\hline atom & 5.64274588 & 5.87450082 & $6.25170911 \mathrm{C}$ \\
\hline atom & 11.72845634 & 5.88256279 & $12.61896314 \mathrm{C}$ \\
\hline atom & 9.57861024 & 20.27154153 & $9.66601312 \mathrm{C}$ \\
\hline atom & 3.49279394 & 20.27960409 & $3.29882268 \mathrm{C}$ \\
\hline atom & 9.48914412 & 8.52065620 & $3.64901193 \mathrm{C}$ \\
\hline atom & 2.86827418 & 8.51871143 & $9.98261491 \mathrm{C}$ \\
\hline atom & 12.35309575 & 22.91581700 & $5.93506894 \mathrm{C}$ \\
\hline atom & 5.73221820 & 22.91766032 & $12.26889978 \mathrm{C}$ \\
\hline atom & 8.26189379 & 6.76876385 & $6.54386016 \mathrm{C}$ \\
\hline atom & 2.16976605 & 6.78140733 & $0.16880929 \mathrm{C}$ \\
\hline atom & 0.87450896 & 21.17834491 & $3.01455211 \mathrm{C}$ \\
\hline atom & 6.95945135 & 21.16573761 & $9.37399352 \mathrm{C}$ \\
\hline atom & 2.69482627 & 7.62989557 & $7.35126582 \mathrm{C}$ \\
\hline atom & 9.32186316 & 7.62974946 & $1.01796156 \mathrm{C}$ \\
\hline atom & -0.18879144 & 22.02675292 & $8.53366811 \mathrm{C}$ \\
\hline atom & 6.43766481 & 22.02678420 & $2.19839913 \mathrm{C}$ \\
\hline atom & 7.95178867 & 5.43555792 & $6.79573613 \mathrm{C}$ \\
\hline atom & 1.86256547 & 5.44790157 & $0.42241845 \mathrm{C}$ \\
\hline atom & 1.18171864 & 19.84485392 & $2.76093078 \mathrm{C}$ \\
\hline atom & 7.26954969 & 19.83255417 & $9.12205599 \mathrm{C}$ \\
\hline atom & 2.42580701 & 8.96170544 & $7.65293405 \mathrm{C}$ \\
\hline atom & 9.05482163 & 8.96244549 & $1.31744612 \mathrm{C}$ \\
\hline atom & 0.08025088 & 23.35858437 & $8.23215952 \mathrm{C}$ \\
\hline atom & 6.70465602 & 23.35950425 & $1.89893140 \mathrm{C}$ \\
\hline atom & -0.10222229 & 5.12068332 & $7.58744556 \mathrm{C}$ \\
\hline atom & 6.52206892 & 5.12000499 & $1.25572988 \mathrm{C}$ \\
\hline atom & 2.60837927 & 19.51778144 & $8.29766188 \mathrm{C}$ \\
\hline atom & 9.23740194 & 19.51706591 & $1.96052989 \mathrm{C}$ \\
\hline atom & 1.81120813 & 9.26855327 & $2.96215269 \mathrm{C}$ \\
\hline atom & 7.90149332 & 9.27562556 & $9.32816787 \mathrm{C}$ \\
\hline atom & 7.31986757 & 23.67268416 & $6.58979754 \mathrm{C}$ \\
\hline atom & 1.23319690 & 23.66561236 & $0.22143822 \mathrm{C}$ \\
\hline atom & 1.52601984 & 3.38874485 & $6.66375849 \mathrm{C}$ \\
\hline atom & 8.15102316 & 3.38591387 & $0.33681596 \mathrm{C}$ \\
\hline atom & 0.98002959 & 17.78582683 & $9.22125912 \mathrm{C}$ \\
\hline tom & 7.60843385 & 17.78298353 & $2.87952788 \mathrm{C}$ \\
\hline atom & 8.89714520 & 11.01481934 & $7.74957082 \mathrm{C}$ \\
\hline & 2.80217268 & 11.00367416 & $1.37710409 \mathrm{C}$ \\
\hline atom & 0.24210899 & 25.40060586 & $1.80650445 \mathrm{C}$ \\
\hline atom & 6.32436478 & 25.41188696 & $8.16843867 \mathrm{C}$ \\
\hline
\end{tabular}




\begin{tabular}{|c|c|c|c|}
\hline aton & 0.33318839 & 3.67142749 & $7.56457984 \mathrm{C}$ \\
\hline aton & 6.95549170 & 3.67014191 & $1.23383190 \mathrm{C}$ \\
\hline 01 & 2.17294256 & 18.06853458 & $8.32053244 \mathrm{C}$ \\
\hline $\mathrm{m}$ & 8.80392434 & 18.06720860 & $1.98245242 \mathrm{C}$ \\
\hline om & 1.85040102 & 10.71834447 & $2.52915826 \mathrm{C}$ \\
\hline om & 7.94155818 & 10.72600841 & $8.89758720 \mathrm{C}$ \\
\hline $\mathrm{m}$ & 7.27986572 & 25.12306718 & $7.02036376 \mathrm{C}$ \\
\hline on & 1.19397208 & 25.11536924 & $0.65451531 \mathrm{C}$ \\
\hline $\mathrm{n}$ & -0.08136226 & 5.82787451 & $8.79519852 \mathrm{C}$ \\
\hline $\mathrm{m}$ & 6.53997859 & 5.82657817 & $2.46385213 \mathrm{C}$ \\
\hline $\mathrm{m}$ & 2.58747747 & 20.22499339 & $7.08991426 \mathrm{C}$ \\
\hline $\mathrm{m}$ & 9.21955366 & 20.22361315 & $0.75238604 \mathrm{C}$ \\
\hline om & 0.60635361 & 8.55980864 & $2.88835804 \mathrm{C}$ \\
\hline $\mathrm{m}$ & 6.69631439 & 8.56759936 & $5590 \mathrm{C}$ \\
\hline om & 8.52503740 & 22.96466726 & $6.66441735 \mathrm{C}$ \\
\hline & 2.43806530 & 2485 & $2432 \mathrm{C}$ \\
\hline & 12.26437731 & 5.79695803 & $6.44391259 \mathrm{C}$ \\
\hline $\mathrm{m}$ & 6.17817626 & 5.79721279 & $0.07860031 \mathrm{C}$ \\
\hline $\mathrm{m}$ & 9.58126622 & 20.19429845 & $5010 \mathrm{C}$ \\
\hline $\mathrm{m}$ & 3633 & 20.1 & $52 \mathrm{C}$ \\
\hline $\mathrm{m}$ & 4213 & 8.5 & $32 \mathrm{C}$ \\
\hline $\mathrm{m}$ & 2965 & $8.5 \mathrm{c}$ & 9.72 \\
\hline $\mathrm{m}$ & -0.46596345 & 22.98 & $12.52547244 \mathrm{C}$ \\
\hline III & 6.15949737 & 22.99 & $6.19081161 \mathrm{C}$ \\
\hline$n$ & 12.31632896 & 918 & $49 \mathrm{C}$ \\
\hline $\mathrm{m}$ & 6.22516157 & 7.1 & $85 \mathrm{C}$ \\
\hline $\mathrm{m}$ & 2.90 & 21.5 & $11 \mathrm{C}$ \\
\hline $\mathrm{m}$ & 9.53 & 21.5 & $92 \mathrm{C}$ \\
\hline $\mathrm{m}$ & 4582 & 801 & $38 \mathrm{C}$ \\
\hline - & 6.65240398 & 2606 & $9.56738737 \mathrm{C}$ \\
\hline$m$ & 8.56 & 21.6 & $360 \mathrm{C}$ \\
\hline $\mathrm{m}$ & 1.94285840 & 9491 & $8633 \mathrm{C}$ \\
\hline $\mathrm{m}$ & 11.95056185 & & $16 \mathrm{C}$ \\
\hline$n$ & 5.86 & 7.15 & $66 \mathrm{C}$ \\
\hline $\mathrm{m}$ & 8401 & 6861 & $52 \mathrm{C}$ \\
\hline $\mathrm{m}$ & 3.27078689 & 21.55125623 & $3509 \mathrm{C}$ \\
\hline $\mathrm{m}$ & 2.928 & 7.23500848 & $193 \mathrm{C}$ \\
\hline $\mathrm{m}$ & 9.01663310 & 7.241 & $10.03683419 \mathrm{C}$ \\
\hline $\mathrm{n}$ & -0.42171096 & 21.63198653 & $86643 \mathrm{C}$ \\
\hline & 6.20 & 21.6 & $07 \mathrm{C}$ \\
\hline n & 11.9 & 23 & $1 \mathrm{C}$ \\
\hline $\mathrm{m}$ & 5.88637444 & 7.848 & $26 \mathrm{C}$ \\
\hline . & 3.24848331 & 22.24500068 & $8.23312877 \mathrm{C}$ \\
\hline $\mathrm{m}$ & 9.87317133 & 8406 & $166 \mathrm{C}$ \\
\hline $\mathrm{m}$ & 1.72270387 & 6.53978704 & $3.59375907 \mathrm{C}$ \\
\hline$n$ & 7.81091905 & 6.54731384 & $9.96068139 \mathrm{C}$ \\
\hline n & 7.410 & 20.9 & $3344 \mathrm{C}$ \\
\hline$n$ & 6 & 20. & $98 \mathrm{C}$ \\
\hline I & 0.170 & 3.55 & $5596 \mathrm{C}$ \\
\hline & 6.25568781 & 1776 & $6.96587690 \mathrm{C}$ \\
\hline 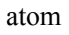 & 8.96564442 & 17.94480192 & $8.95182438 \mathrm{C}$ \\
\hline $\mathrm{m}$ & 2.87425018 & 17.95440346 & $2.58274131 \mathrm{C}$ \\
\hline $\mathrm{n}$ & 2.17320771 & 10.84437610 & $9.34482757 \mathrm{C}$ \\
\hline & 8.80256540 & 10.84777231 & $3.00619370 \mathrm{C}$ \\
\hline & 0.33296240 & 25.2 & $6.54039529 \mathrm{C}$ \\
\hline & 6.95685220 & 25.24488344 & $0.21021666 \mathrm{C}$ \\
\hline & 0.65725966 & 3.06185761 & $1.94948317 \mathrm{C}$ \\
\hline & 6.72886484 & 3.05034621 & $8.31913614 \mathrm{C}$ \\
\hline $\mathrm{m}$ & 8.49246626 & 17.44746588 & $7.59854288 \mathrm{C}$ \\
\hline & 2.38708293 & 17.45883990 & $1.23396208 \mathrm{C}$ \\
\hline & 0.84473293 & $11.33 \mathrm{~S}$ & $7269 \mathrm{C}$ \\
\hline & 7.47898607 & 11.34901982 & $2.45909835 \mathrm{C}$ \\
\hline & 1.66139496 & 25.73697971 & $7.08047203 \mathrm{C}$ \\
\hline & 8.28045954 & 25.74615586 & $0.75724974 \mathrm{C}$ \\
\hline & 5.16110291 & 7.91996857 & $5.76146133 \mathrm{H}$ \\
\hline $\mathrm{n}$ & 11.24199455 & 7.92669275 & $12.12785662 \mathrm{H}$ \\
\hline & 10.06027796 & 22.31700240 & $10.15628148 \mathrm{H}$ \\
\hline & 3.97924023 & 22.32372899 & $3.78999238 \mathrm{H}$ \\
\hline & 9.95990292 & 6.47544215 & $4.15054826 \mathrm{H}$ \\
\hline & 3.34287397 & 6.47374616 & $10.48107553 \mathrm{H}$ \\
\hline
\end{tabular}




\begin{tabular}{|c|c|c|c|}
\hline atom & 11.87845301 & 20.87090268 & $5.43638048 \mathrm{H}$ \\
\hline atom & 5.26152982 & 20.87242805 & $11.76734498 \mathrm{H}$ \\
\hline atom & 7.48282032 & 8.70959799 & $5.99275301 \mathrm{H}$ \\
\hline tom & 0.84823764 & 8.72019984 & $12.31825125 \mathrm{H}$ \\
\hline tom & 1.65785732 & 23.11721400 & $3.56673861 \mathrm{H}$ \\
\hline om & 7.73853652 & 23.10656865 & $9.92512811 \mathrm{H}$ \\
\hline atom & 3.21687002 & 5.68888059 & $8.14965161 \mathrm{H}$ \\
\hline atom & 9.83908931 & 5.68877226 & $1.81942731 \mathrm{H}$ \\
\hline atom & 12.00438998 & 20.08583867 & $7.76785498 \mathrm{H}$ \\
\hline atom & 5.92052166 & 20.08579277 & $1.39689732 \mathrm{H}$ \\
\hline atom & 4.62205304 & 5.51575986 & $6.12601104 \mathrm{H}$ \\
\hline atom & 10.70792678 & 5.52226220 & $12.49695767 \mathrm{H}$ \\
\hline atom & 10.59930372 & 19.91281576 & $9.79166634 \mathrm{H}$ \\
\hline atom & 4.51331784 & 19.91933116 & $3.42088067 \mathrm{H}$ \\
\hline atom & 9.56520723 & 8.87784185 & $4.67505858 \mathrm{H}$ \\
\hline atom & 2.94718379 & 8.87529848 & $11.00863566 \mathrm{H}$ \\
\hline atom & 12.27423107 & 23.27249077 & $4.90907365 \mathrm{H}$ \\
\hline atom & 5.65615195 & 23.27486521 & $11.24285664 \mathrm{H}$ \\
\hline atom & 9.28325478 & 7.11534023 & $6.68858720 \mathrm{H}$ \\
\hline atom & 3.19095951 & 7.12970126 & $0.31071757 \mathrm{H}$ \\
\hline atom & -0.14666848 & 21.52662809 & $2.87256727 \mathrm{H}$ \\
\hline atom & 5.93808194 & 21.51229138 & $9.22933833 \mathrm{H}$ \\
\hline atom & 2.59620185 & 7.28535151 & $6.32374768 \mathrm{H}$ \\
\hline atom & 9.22543024 & 7.28425163 & $-0.00944487 \mathrm{H}$ \\
\hline atom & -0.09021987 & 21.68195762 & $9.56110798 \mathrm{H}$ \\
\hline atom & 6.53411155 & 21.68126773 & $3.22580058 \mathrm{H}$ \\
\hline atom & 8.74088274 & 4.76414903 & $7.13532894 \mathrm{H}$ \\
\hline atom & 2.65372084 & 4.77811564 & $0.76024253 \mathrm{H}$ \\
\hline atom & 0.39058268 & 19.17506354 & $2.42303397 \mathrm{H}$ \\
\hline atom & 6.48043276 & 19.16113143 & $8.78250781 \mathrm{H}$ \\
\hline atom & 2.11932161 & 9.63391721 & $6.85115440 \mathrm{H}$ \\
\hline atom & 8.75265844 & 9.63473436 & $0.51405241 \mathrm{H}$ \\
\hline atom & 0.38671472 & 24.03077187 & $9.03396529 \mathrm{H}$ \\
\hline atom & 7.00678260 & 24.03179844 & $2.70233417 \mathrm{H}$ \\
\hline atom & 11.46726732 & 3.01680752 & $6.27698209 \mathrm{H}$ \\
\hline atom & 4.83561984 & 3.02116928 & $12.61286961 \mathrm{H}$ \\
\hline atom & 10.38564643 & 17.41826394 & $3.30491631 \mathrm{H}$ \\
\hline atom & 3.75400040 & 17.41397249 & $9.64087572 \mathrm{H}$ \\
\hline atom & 3.09911738 & 11.37695973 & $4.16619074 \mathrm{H}$ \\
\hline atom & 9.18527249 & 11.38219573 & $10.53890214 \mathrm{H}$ \\
\hline atom & 12.12234867 & 25.77400082 & $11.75169632 \mathrm{H}$ \\
\hline atom & 6.03608039 & 25.77921674 & $5.37909480 \mathrm{H}$ \\
\hline atom & 11.15313998 & 2.82648947 & $7.93370761 \mathrm{H}$ \\
\hline atom & 5.05950877 & 2.82556394 & $1.56755627 \mathrm{H}$ \\
\hline atom & 4.06810693 & 17.22352790 & $7.98417526 \mathrm{H}$ \\
\hline atom & 10.69987009 & 17.22262265 & $1.64867627 \mathrm{H}$ \\
\hline atom & 1.42945713 & 11.56026461 & $4.40862667 \mathrm{H}$ \\
\hline atom & 7.51445920 & 11.56222988 & $10.77823445 \mathrm{H}$ \\
\hline atom & 7.70688232 & 25.95928752 & $5.13969182 \mathrm{H}$ \\
\hline atom & 1.07684592 & 25.95728989 & $11.47663232 \mathrm{H}$ \\
\hline atom & 12.20395125 & 1.78816206 & $7.14361168 \mathrm{H}$ \\
\hline atom & 6.10793338 & 1.78904379 & $0.77377180 \mathrm{H}$ \\
\hline atom & 3.01723452 & 16.18528570 & $8.77429114 \mathrm{H}$ \\
\hline atom & 9.65141411 & 16.18609557 & $2.44244286 \mathrm{H}$ \\
\hline atom & 2.25973907 & 12.60262138 & $3.39374754 \mathrm{H}$ \\
\hline atom & 8.34562084 & 12.60828364 & $9.76869442 \mathrm{H}$ \\
\hline atom & 6.87575701 & 27.00532800 & $6.14926462 \mathrm{H}$ \\
\hline atom & 0.24658860 & 26.99962022 & $12.49152493 \mathrm{H}$ \\
\hline atom & 1.86968345 & 2.35199292 & $6.76798984 \mathrm{H}$ \\
\hline atom & 8.49396057 & 2.34915723 & $0.44379555 \mathrm{H}$ \\
\hline atom & 0.63640213 & 16.74905375 & $9.11703169 \mathrm{H}$ \\
\hline atom & 7.26545414 & 16.74623180 & $2.77256941 \mathrm{H}$ \\
\hline atom & 8.80576580 & 12.05247530 & $7.40465680 \mathrm{H}$ \\
\hline atom & 2.70484538 & 12.03824520 & $1.02495621 \mathrm{H}$ \\
\hline atom & 0.33940325 & 26.43516553 & $2.15868863 \mathrm{H}$ \\
\hline atom & 6.41576390 & 26.44956176 & $8.51329130 \mathrm{H}$ \\
\hline atom & 2.35314882 & 4.04657530 & $6.94820960 \mathrm{H}$ \\
\hline atom & 8.97771131 & 4.04335538 & $0.62335328 \mathrm{H}$ \\
\hline atom & 0.15291284 & 18.44360273 & $8.93669744 \mathrm{H}$ \\
\hline atom & 6.78175305 & 18.44043272 & $2.59303860 \mathrm{H}$ \\
\hline
\end{tabular}




\begin{tabular}{|c|c|c|c|}
\hline atom & 8.65410802 & 10.35922100 & $6.90758346 \mathrm{H}$ \\
\hline atom & 2.56058721 & 10.34089776 & $0.54043783 \mathrm{H}$ \\
\hline atom & 0.48361418 & 24.73780245 & $2.64317076 \mathrm{H}$ \\
\hline atom & 6.56746791 & 24.75633433 & $9.01044413 \mathrm{H}$ \\
\hline atom & 1.29852680 & 3.56944845 & $5.60621860 \mathrm{H}$ \\
\hline atom & 7.38922235 & 3.56576437 & $11.97986022 \mathrm{H}$ \\
\hline atom & 1.20741121 & 17.96657264 & $10.27881597 \mathrm{H}$ \\
\hline atom & 7.83215590 & 17.96290047 & $3.93801602 \mathrm{H}$ \\
\hline atom & 9.94330583 & 10.83618601 & $8.02609140 \mathrm{H}$ \\
\hline atom & 3.84964007 & 10.83130759 & $1.65270934 \mathrm{H}$ \\
\hline atom & 11.90982889 & 25.22824658 & $1.56347962 \mathrm{H}$ \\
\hline atom & 5.27818341 & 25.23321067 & $7.89201042 \mathrm{H}$ \\
\hline atom & 0.57536458 & 3.36269793 & $8.59043786 \mathrm{H}$ \\
\hline atom & 7.19420395 & 3.36102033 & $2.26037934 \mathrm{H}$ \\
\hline atom & 1.93082910 & 17.75979341 & $7.29466306 \mathrm{H}$ \\
\hline atom & 8.56514992 & 17.75811001 & $0.95591401 \mathrm{H}$ \\
\hline atom & 0.83559480 & 11.02514316 & $2.24211625 \mathrm{H}$ \\
\hline atom & 6.92753661 & 11.03276067 & $8.60772359 \mathrm{H}$ \\
\hline atom & 8.29391304 & 25.42983001 & $7.31014865 \mathrm{H}$ \\
\hline atom & 2.20875567 & 25.42218465 & $0.94164184 \mathrm{H}$ \\
\hline atom & 0.21440230 & 5.32290157 & $9.71484459 \mathrm{H}$ \\
\hline atom & 6.83161942 & 5.32058468 & $3.38428001 \mathrm{H}$ \\
\hline atom & 2.29167482 & 19.72002521 & $6.17027813 \mathrm{H}$ \\
\hline atom & 8.38975085 & 19.71753080 & $12.53351372 \mathrm{H}$ \\
\hline atom & 12.41468271 & 9.06439082 & $2.58734857 \mathrm{H}$ \\
\hline atom & 5.79021667 & 9.07247688 & $8.91831661 \mathrm{H}$ \\
\hline atom & 9.43117385 & 23.46953816 & $6.99949268 \mathrm{H}$ \\
\hline atom & 3.34488783 & 23.46151689 & $0.62894021 \mathrm{H}$ \\
\hline atom & 12.24942532 & 5.27004569 & $5.48940172 \mathrm{H}$ \\
\hline atom & 5.62771464 & 5.27078773 & $11.82525445 \mathrm{H}$ \\
\hline atom & 9.59352023 & 19.66797491 & $4.09256045 \mathrm{H}$ \\
\hline atom & 2.97202933 & 19.66708327 & $10.42836385 \mathrm{H}$ \\
\hline atom & 10.01449855 & 9.12726981 & $9.78473953 \mathrm{H}$ \\
\hline atom & 3.92464492 & 9.12092170 & $3.41637653 \mathrm{H}$ \\
\hline atom & 5.20683714 & 23.52433272 & $6.13333406 \mathrm{H}$ \\
\hline atom & 11.29679393 & 23.51785407 & $12.50135234 \mathrm{H}$ \\
\hline atom & -0.34168603 & 7.72382778 & $9.76843850 \mathrm{H}$ \\
\hline atom & 6.28041480 & 7.72300138 & $3.43677780 \mathrm{H}$ \\
\hline atom & 2.84773551 & 22.12097190 & $6.11667829 \mathrm{H}$ \\
\hline atom & 8.94103601 & 22.11994119 & $12.48095662 \mathrm{H}$ \\
\hline atom & 12.34016965 & 6.66215926 & $3.13604197 \mathrm{H}$ \\
\hline atom & 5.71385929 & 6.67054752 & $9.46827338 \mathrm{H}$ \\
\hline atom & 9.50744928 & 21.06764142 & $6.44944705 \mathrm{H}$ \\
\hline atom & 3.41947146 & 21.05933283 & $0.08011296 \mathrm{H}$ \\
\hline atom & 11.72556732 & 7.68366879 & $5.55289037 \mathrm{H}$ \\
\hline atom & 5.10682416 & 7.68504883 & $11.88820731 \mathrm{H}$ \\
\hline atom & 10.11447717 & 22.08222852 & $4.02954686 \mathrm{H}$ \\
\hline atom & 3.49582754 & 22.08072488 & $10.36488179 \mathrm{H}$ \\
\hline atom & 3.84152335 & 6.70603416 & $3.93268317 \mathrm{H}$ \\
\hline atom & 9.92969810 & 6.71237986 & $10.30080150 \mathrm{H}$ \\
\hline atom & 11.37997658 & 21.10299818 & $11.98491628 \mathrm{H}$ \\
\hline atom & 5.29155495 & 21.10947280 & $5.61718374 \mathrm{H}$ \\
\hline atom & 11.74896558 & 8.91339615 & $7.69363926 \mathrm{H}$ \\
\hline atom & 5.66444383 & 8.91414094 & $1.32788103 \mathrm{H}$ \\
\hline atom & 3.47229366 & 23.31050882 & $8.22415723 \mathrm{H}$ \\
\hline atom & 10.09512700 & 23.31120121 & $1.88829161 \mathrm{H}$ \\
\hline atom & 1.70533743 & 5.47394205 & $3.81552085 \mathrm{H}$ \\
\hline atom & 7.79302710 & 5.48137034 & $10.18196163 \mathrm{H}$ \\
\hline atom & 7.42822967 & 19.87847867 & $5.73583456 \mathrm{H}$ \\
\hline atom & 0.80102787 & 19.87099053 & $12.06940504 \mathrm{H}$ \\
\hline atom & 5.16295364 & 3.44911936 & $6.90611790 \mathrm{H}$ \\
\hline atom & 11.79196889 & 3.45843458 & $0.58425474 \mathrm{H}$ \\
\hline atom & 10.05837944 & 17.84620444 & $9.01157798 \mathrm{H}$ \\
\hline atom & 3.96749764 & 17.85548730 & $2.63188326 \mathrm{H}$ \\
\hline atom & 8.80095157 & 10.94273189 & $4.10090762 \mathrm{H}$ \\
\hline atom & 2.17671541 & 10.93849278 & $10.43959551 \mathrm{H}$ \\
\hline atom & 0.32952746 & 25.33559525 & $5.44563531 \mathrm{H}$ \\
\hline atom & 6.42024073 & 25.33980546 & $11.81705083 \mathrm{H}$ \\
\hline atom & 7.74562621 & 2.83235360 & $5.57749699 \mathrm{H}$ \\
\hline atom & 1.11120322 & 2.83591324 & $11.90599358 \mathrm{H}$ \\
\hline
\end{tabular}




\begin{tabular}{|c|c|c|c|}
\hline tom & 1.39489682 & 17.23283703 & $3.97889996 \mathrm{H}$ \\
\hline ton & 7.47569510 & 17.22931095 & $10.34015504 \mathrm{H}$ \\
\hline om & 3.62430012 & 11.57956978 & $7.92687698 \mathrm{H}$ \\
\hline III & 10.26499221 & 11.57351051 & $1.59441273 \mathrm{H}$ \\
\hline $\mathrm{m}$ & 11.59700697 & 25.97651516 & $7.99099910 \mathrm{H}$ \\
\hline $\mathrm{m}$ & 5.49439021 & 25.97043530 & $1.62204400 \mathrm{H}$ \\
\hline & -0.48915766 & 2.75267919 & $11.36522894 \mathrm{H}$ \\
\hline & 6.14832758 & 2.74366470 & $5.02909287 \mathrm{H}$ \\
\hline & 2.99522837 & 17.14976903 & $4.51978104 \mathrm{H}$ \\
\hline & 9.07298274 & 17.14063288 & $10.88858103 \mathrm{H}$ \\
\hline & 10.73595244 & 11.64180275 & $3.21732487 \mathrm{H}$ \\
\hline & 4.10400136 & 11.64623326 & $9.54689949 \mathrm{H}$ \\
\hline $\mathrm{m}$ & 11.11731095 & 26.04311299 & $6.37093681 \mathrm{H}$ \\
\hline $\mathrm{m}$ & 5.02349849 & 26.03891428 & $50 \mathrm{H}$ \\
\hline & 6.70592528 & 1.643 & 6.155 \\
\hline & 0.069 & 1.650 & 12.4 \\
\hline & 2.43641607 & 16.04803312 & $3.39380019 \mathrm{H}$ \\
\hline & 8.51540955 & $16 .($ & $221 \mathrm{H}$ \\
\hline & 3.00021914 & 42963 & $847 \mathrm{H}$ \\
\hline $\mathrm{m}$ & 9.64 & 12.7 & $16 \mathrm{H}$ \\
\hline $\mathrm{m}$ & 12.22 & 0108 & $94 \mathrm{H}$ \\
\hline $\mathrm{m}$ & 6.11807766 & 5885 & $41 \mathrm{H}$ \\
\hline & 1.74765409 & 3.10 & $2.04380407 \mathrm{H}$ \\
\hline & $7.818-r=2$ & 207 & 8.423 \\
\hline & 77590 & 17. & $73 \mathrm{H}$ \\
\hline & 1.29 & 17. & $72 \mathrm{H}$ \\
\hline $\mathrm{m}$ & 0.79 & 11. & $10 \mathrm{H}$ \\
\hline $\mathrm{m}$ & 7.4 & 11. & $2 \mathrm{H}$ \\
\hline $\mathrm{m}$ & 1.70814961 & 25. & $258 \mathrm{H}$ \\
\hline $\mathrm{m}$ & 8.32419497 & 25.7 & $504 \mathrm{H}$ \\
\hline $\mathrm{m}$ & 0.33 & & $7 \mathrm{H}$ \\
\hline & 9754 & 4888 & $207 \mathrm{H}$ \\
\hline & 8.8 & 6883 & $98 \mathrm{H}$ \\
\hline 1 & 2.71 & 16. & $2 \mathrm{H}$ \\
\hline $\mathrm{m}$ & 0.64 & 12. & $882 \mathrm{H}$ \\
\hline $\mathrm{m}$ & 7.283 & 3869 & $094 \mathrm{H}$ \\
\hline $\mathrm{m}$ & 1.86 & 26.7 & $31 \mathrm{H}$ \\
\hline $\mathrm{m}$ & 8.47560530 & 26.77621621 & $0641 \mathrm{H}$ \\
\hline 1 & 0.22206308 & 3.68889066 & $2.73544245 \mathrm{H}$ \\
\hline $\mathrm{n}$ & 6.288 & 3.6 & $307 \mathrm{H}$ \\
\hline & 8.93 & 18. & $6 \mathrm{H}$ \\
\hline $\mathrm{m}$ & 2.822 & $18 .($ & $69 \mathrm{H}$ \\
\hline & 0.04357303 & 8096 & $2602 \mathrm{H}$ \\
\hline $\mathrm{m}$ & 6.672 & 10.7 & $993 \mathrm{H}$ \\
\hline 1 & 2.46257755 & 25.10209467 & $6.68635802 \mathrm{H}$ \\
\hline 1 & 9.08736977 & 25.11 & $5644 \mathrm{H}$ \\
\hline & 11.89 & & $7272 \mathrm{~N}$ \\
\hline & 5.80 & 2.77 & 0.83 \\
\hline & 3.326 & 17.16 & $541 \mathrm{~N}$ \\
\hline & 9.957 & 0755 & $154 \mathrm{~N}$ \\
\hline & 2.19757432 & 11.61760717 & $3.69906369 \mathrm{~N}$ \\
\hline 1 & 8.28429023 & 11.62214853 & $10.07073052 \mathrm{~N}$ \\
\hline & 6.93708376 & 26.01919702 & $5.84722579 \mathrm{~N}$ \\
\hline & 0.30872320 & 26.01461660 & $12.18617522 \mathrm{~N}$ \\
\hline & 6.76486980 & 8051 & $5.86676716 \mathrm{~N}$ \\
\hline & 0.13102208 & 2.64066517 & $12.20010899 \mathrm{~N}$ \\
\hline & 2.37510768 & 17.03769411 & $335 \mathrm{~N}$ \\
\hline & 8.45645090 & 17.03056894 & $10.05089255 \mathrm{~N}$ \\
\hline & 3.29341589 & 11.76524155 & $8.89667774 \mathrm{~N}$ \\
\hline & 9.92929516 & 11.76227670 & $2.56224826 \mathrm{~N}$ \\
\hline & 11.92786742 & 26.16219760 & $7.02118937 \mathrm{~N}$ \\
\hline & 5.83012857 & 26.15932310 & $0.65424202 \mathrm{~N}$ \\
\hline
\end{tabular}
$(S-M B A)_{2} \mathrm{~Pb}_{1-x} \mathrm{Sn}_{x} \mathrm{I}_{4}(\mathrm{x}=0.5)$ default setting
$\mathbf{c}(\mathbf{2} \times \mathbf{2})$ unit cel1, PBE+TS relaxed with "tight"

lattice_vector $\quad 8.80472416 \quad-0.00352576 \quad-0.00000416$ 
$\begin{array}{llll}\text { lattice_vector } & -0.00372599 & 28.77994953 & 0.00010226\end{array}$

lattice_vector $\quad-0.00000530 \quad-0.00012930 \quad 9.15562303$

$\begin{array}{llll}\text { atom } & 3.19004393 & 7.22589013 & 2.22103516 \mathrm{~Pb}\end{array}$

atom $\quad \begin{array}{lll}1.20843278 & 21.55202362 & 6.79905633 \mathrm{~Pb}\end{array}$

atom $\quad 5.61069339 \quad 21.62053092 \quad 2.28871611 \mathrm{Sn}$

atom $\quad 7.59236722 \quad 7.15408615 \quad 6.86629892 \mathrm{Sn}$

$\begin{array}{llll}\text { atom } \quad 3.30680548 & 4.01627927 & 2.56824230 \mathrm{I}\end{array}$

$\begin{array}{llll}\text { atom } & 1.09180914 & 24.76197195 & 7.14610709 \mathrm{I}\end{array}$

$\begin{array}{llll}\text { atom } & 5.48828458 & 18.44827973 & 2.01191155 \mathrm{I}\end{array}$

atom $\quad \begin{array}{llll}7.71512348 & 10.32630332 & 6.58965688 \mathrm{I}\end{array}$

$\begin{array}{llll}\text { atom } & 3.26146030 & 10.42150723 & 2.53909053 \mathrm{I}\end{array}$

atom $\quad \begin{array}{llll}1.13717110 & 18.35653264 & 7.11699763 \mathrm{I}\end{array}$

$\begin{array}{llll}\text { atom } & 5.53695170 & 24.77218575 & 2.03605289 \mathrm{I}\end{array}$

$\begin{array}{llll}\text { atom } & 7.66642973 & 4.00236876 & 6.61376368 \mathrm{I}\end{array}$

$\begin{array}{llll}\text { atom } & 5.93676064 & 7.21103320 & 0.61404564 \mathrm{I}\end{array}$

atom $\quad 7.26653853 \quad 21.56361220 \quad 5.19198625 \mathrm{I}$

$\begin{array}{llll}\text { atom } & 2.91481131 & 21.60864452 & 3.90973724 \mathrm{I}\end{array}$

$\begin{array}{llll}\text { atom } & 1.48393548 & 7.16944307 & 8.48760272 \mathrm{I}\end{array}$

$\begin{array}{llll}\text { atom } & 4.82558867 & 7.19047404 & 5.08456462 \mathrm{I}\end{array}$

$\begin{array}{llll}\text { atom } & 8.37772724 & 21.58415296 & 0.50693146 \mathrm{I}\end{array}$

$\begin{array}{llll}\text { atom } & 4.00551994 & 21.58681122 & 8.67111209 \mathrm{I}\end{array}$

$\begin{array}{llll}\text { atom } & 0.39322157 & 7.19126542 & 4.09315543 \mathrm{I}\end{array}$

$\begin{array}{llll}\text { atom } & 6.71000219 & 4.55426259 & 3.11574829 \mathrm{~N}\end{array}$

$\begin{array}{llll}\text { atom } & 6.49336087 & 24.22025312 & 7.69363910 \mathrm{~N}\end{array}$

$\begin{array}{llll}\text { atom } & 2.07803793 & 18.95264958 & 1.44672888 \mathrm{~N}\end{array}$

$\begin{array}{llll}\text { atom } & 2.32059163 & 9.82541286 & 6.02449081 \mathrm{~N}\end{array}$

$\begin{array}{llll}\text { atom } & 6.80918368 & 9.96639882 & 3.23988158 \mathrm{~N}\end{array}$

$\begin{array}{llll}\text { atom } & 6.39419271 & 18.80809286 & 7.81774191 \mathrm{~N}\end{array}$

$\begin{array}{llll}\text { atom } & 1.97938899 & 24.34985085 & 1.33423343 \mathrm{~N}\end{array}$

$\begin{array}{llll}\text { atom } & 2.41926129 & 4.42825520 & 5.91194621 \mathrm{~N}\end{array}$

$\begin{array}{llll}\text { atom } & 6.86554431 & 5.54350792 & 2.86047740 \mathrm{H}\end{array}$

$\begin{array}{llll}\text { atom } & 6.33783422 & 23.23101445 & 7.43835487 \mathrm{H}\end{array}$

$\begin{array}{llll}\text { atom } & 1.90683282 & 19.94276319 & 1.68737672 \mathrm{H}\end{array}$

$\begin{array}{llll}\text { atom } & 2.49176141 & 8.83528863 & 6.26515134 \mathrm{H}\end{array}$

atom $\quad 7.18118995 \quad 4.37204314 \quad 4.02657581 \mathrm{H}$

atom $\quad \begin{array}{llll}6.02217044 & 24.40243907 & 8.60446455 \mathrm{H}\end{array}$

atom $\quad \begin{array}{llll}1.61266924 & 18.75181299 & 0.53651158 \mathrm{H}\end{array}$

atom $\quad 2.78599482 \quad 10.02625113 \quad 5.11428799 \mathrm{H}$

atom $\quad 5.67981190 \quad 4.43335228 \quad 3.25099366 \mathrm{H}$

$\begin{array}{llll}\text { atom } & 7.52354869 & 24.34117335 & 7.82889006 \mathrm{H}\end{array}$

$\begin{array}{llll}\text { atom } & 3.11007635 & 18.84433426 & 1.31610946 \mathrm{H}\end{array}$

atom $\quad \begin{array}{llll}1.28856273 & 9.93375687 & 5.89384052 \mathrm{H}\end{array}$

$\begin{array}{llll}\text { atom } & 5.84780431 & 10.21658731 & 2.91986544 \mathrm{H}\end{array}$

atom $\quad 7.35557420 \quad 18.55792133 \quad 7.49772496 \mathrm{H}$

$\begin{array}{llll}\text { atom } & 2.94122410 & 24.59025817 & 1.65930882 \mathrm{H}\end{array}$

$\begin{array}{llll}\text { atom } & 1.45742040 & 4.18781271 & 6.23698207 \mathrm{H}\end{array}$

$\begin{array}{llll}\text { atom } & 6.83384954 & 10.01086305 & 4.28586614 \mathrm{H}\end{array}$

$\begin{array}{llll}\text { atom } & 6.36952184 & 18.76359149 & 8.86372605 \mathrm{H}\end{array}$

$\begin{array}{llll}\text { atom } & 1.95736492 & 24.40731173 & 0.28874966 \mathrm{H}\end{array}$

atom $\quad 2.44131590 \quad 4.37087174 \quad 4.86645893 \mathrm{H}$

$\begin{array}{llll}\text { atom } & 6.97710615 & 8.98347010 & 2.97027945 \mathrm{H}\end{array}$

$\begin{array}{llll}\text { atom } & 6.22626617 & 19.79103123 & 7.54814255 \mathrm{H}\end{array}$

atom $\quad \begin{array}{lll}1.80416562 & 23.36481435 & 1.59156075 \mathrm{H}\end{array}$

atom $\quad 2.59445928 \quad 5.41327328 \quad 6.16932611 \mathrm{H}$

atom $\quad \begin{array}{llll}7.06722685 & 16.10935457 & 3.68743710 \mathrm{H}\end{array}$

atom $\quad \begin{array}{llll}6.13609583 & 12.66510283 & 8.26521862 \mathrm{H}\end{array}$

$\begin{array}{llll}\text { atom } & 1.74403829 & 1.71057465 & 0.89909296 \mathrm{H}\end{array}$

atom $\quad 2.65457734 \quad 27.06754098 \quad 5.47698645 \mathrm{H}$

$\begin{array}{llll}\text { atom } & 5.50489740 & 14.87928959 & 2.22432040 \mathrm{H}\end{array}$

$\begin{array}{llll}\text { atom } & 7.69844564 & 13.89521375 & 6.80213173 \mathrm{H}\end{array}$

$\begin{array}{llll}\text { atom } & 3.30262283 & 0.47514003 & 2.36108002 \mathrm{H}\end{array}$

$\begin{array}{llll}\text { atom } & 1.09600966 & 28.30293265 & 6.93899458 \mathrm{H}\end{array}$

atom $\quad 7.29099764 \quad 27.25582035 \quad 3.57566940 \mathrm{H}$

$\begin{array}{llll}\text { atom } & 5.91233422 & 1.51866606 & 8.15338643 \mathrm{H}\end{array}$

$\begin{array}{llll}\text { atom } & 1.51540808 & 12.87604433 & 1.01472075 \mathrm{H}\end{array}$

$\begin{array}{llll}\text { atom } & 2.88321945 & 15.90204189 & 5.59255379 \mathrm{H}\end{array}$

atom $\quad 5.52204921 \quad 28.04581945 \quad 2.05384276 \mathrm{H}$

$\begin{array}{llll}\text { atom } & 7.68128583 & 0.72873190 & 6.63155733 \mathrm{H}\end{array}$

atom $\quad 3.28034962 \quad 13.67396980 \quad 2.53597454 \mathrm{H}$

atom $\quad 1.11828474 \quad 15.10405452 \quad 7.11380541 \mathrm{H}$ 


\begin{tabular}{|c|c|c|c|}
\hline and & 0.21482390 & 14.92304900 & \\
\hline & 4.18379423 & 13.85504488 & $0.11879205 \mathrm{H}$ \\
\hline & 8.59040084 & 0.52432644 & $9.04791981 \mathrm{H}$ \\
\hline & 4.61293258 & 28.25015830 & $4.47019367 \mathrm{H}$ \\
\hline & 8.37806980 & 5.15569985 & $1.04499732 \mathrm{H}$ \\
\hline & 4.82529032 & 23.61887441 & 5.622 \\
\hline & 0.41735046 & 19.56501927 & \\
\hline & 3.98129980 & 9.21296564 & 8.0971 \\
\hline & 8.74835652 & 3.4 & 0.567 \\
\hline & 4.45499475 & 25.28005630 & 5.14529 \\
\hline & 0.06234889 & 17.90695091 & \\
\hline & 4.33627410 & 10.87101197 & 8.5 \\
\hline & 0.489 & & 21434 \\
\hline & 3.90 & 24.6754 & 6.721 \\
\hline & 8.303 & & \\
\hline & 4.89 & 10.2 & 7.0 \\
\hline & & & 1.02 \\
\hline & 4.81 & 19.2 & 5.6 \\
\hline & & 23. & \\
\hline & 3.99 & 4.8 & 8.13 \\
\hline & & 11.2 & \\
\hline & 4.3 & 17.5 & \\
\hline & -0.0 & 25.6 & 3.7 \\
\hline $\mathrm{m}$ & & & \\
\hline & 7.1 & 10.7 & \\
\hline & & & \\
\hline & 1.6 & 25 . & \\
\hline & 2.74 & & \\
\hline & 5.42 & 0 & 1.4 \\
\hline & 7.77 & 27.1 & \\
\hline & 3.37 & 16. & \\
\hline & & 12. & \\
\hline & 5.82 & 12. & \\
\hline & & & \\
\hline & 2.97 & 26. & \\
\hline & 1.42 & & \\
\hline & & & \\
\hline & 4.1 & 28. & \\
\hline $\mathrm{m}$ & & & \\
\hline & 4.6 & 14. & 4.7 \\
\hline & 0.5 & & \\
\hline & 3.8 & 16. & \\
\hline & 8.23 & 26. & \\
\hline & 4.9 & 1.8 & 4.9 \\
\hline & & & 3.6 \\
\hline & 4.20 & 26.3 & 8.2 \\
\hline & -0.1 & 16. & \\
\hline & 97 & 11.9 & \\
\hline & & 10. & \\
\hline & & & \\
\hline & -0.01 & 24. & 1.3 \\
\hline & & & \\
\hline & 3745 & 3.7 & 1.24 \\
\hline & & & \\
\hline & 9168 & 18. & \\
\hline & 1.97 & 10. & 7.9 \\
\hline & 7.214 & 15.0 & \\
\hline & 5.98 & 13. & 8.0 \\
\hline & 1.592 & 0.6 & 1.06 \\
\hline & 2.80 & 28.1 & 5.6 \\
\hline & 6.32 & 14.3 & 2.70 \\
\hline & 6.87669123 & 14.42495484 & 7.2794 \\
\hline & 2.474 & 28.72817 & 1.884 \\
\hline & 1.92363511 & 0.04990969 & 6.46227 \\
\hline & & 28.30661641 & \\
\hline & 5.94166288 & 0.46788047 & 7.8708 \\
\hline & & & \\
\hline & 2.85770538 & 14.85066648 & 5.873 \\
\hline & & -0.02892558 & $2.43987873 \mathrm{C}$ \\
\hline & 6.94347173 & 28.80345871 & 7.01779390 \\
\hline
\end{tabular}




\begin{tabular}{|c|c|c|c|}
\hline atom & 2.54464956 & 14.37648470 & $2.14817597 \mathrm{C}$ \\
\hline aton & 1.85398074 & 14.40155484 & $6.72599281 \mathrm{C}$ \\
\hline om & 8.29943604 & 14.37909474 & $4.08644269 \mathrm{C}$ \\
\hline atom & 4.90390952 & 14.39536207 & $8.66425011 \mathrm{C}$ \\
\hline atom & 0.50089499 & 28.76505328 & $0.50182780 \mathrm{C}$ \\
\hline atom & 3.89772706 & 0.01306922 & $5.07952385 \mathrm{C}$ \\
\hline atom & 8.47488029 & 4.12910126 & $1.41756518 \mathrm{C}$ \\
\hline $\mathrm{m}$ & 4.72847720 & 24.64545882 & $5.99545798 \mathrm{C}$ \\
\hline atom & 0.32473748 & 18.53346700 & $3.15969776 \mathrm{C}$ \\
\hline om & 4.07389402 & 10.24453891 & $7.73745930 \mathrm{C}$ \\
\hline om & 8.06903713 & 10.58622925 & $1.20020790 \mathrm{C}$ \\
\hline atom & 5.13434386 & 18.18833083 & $5.77805893 \mathrm{C}$ \\
\hline atom & 0.72751180 & 24.96209736 & $3.38078213 \mathrm{C}$ \\
\hline om & 3.67112730 & 3.81593015 & $7.95849895 \mathrm{C}$ \\
\hline om & 6.21814321 & 1.30949053 & $2.05890601 \mathrm{C}$ \\
\hline om & 6.98520490 & 27.46505653 & $6.63681487 \mathrm{C}$ \\
\hline $\mathrm{m}$ & 2.58327869 & 15.71596945 & $2.52558975 \mathrm{C}$ \\
\hline atom & 1.81535069 & 13.06205378 & $7.10338947 \mathrm{C}$ \\
\hline atom & 7.19524028 & 2.20670350 & $2.49753480 \mathrm{C}$ \\
\hline om & 6.00811110 & 26.56782530 & $7.07543561 \mathrm{C}$ \\
\hline $\mathrm{m}$ & 1.60416512 & 16.60929338 & $5665 \mathrm{C}$ \\
\hline om & 2.79445932 & 12.16874661 & $6.66143955 \mathrm{C}$ \\
\hline om & 7.60943050 & 12.31539339 & $3.01709890 \mathrm{C}$ \\
\hline $\mathrm{m}$ & 5.59394387 & 16.45910433 & $7.59494085 \mathrm{C}$ \\
\hline om & 1.18542798 & 26.69867180 & $1.56965374 \mathrm{C}$ \\
\hline$n$ & 3.21320930 & 2.07941753 & $6.14736156 \mathrm{C}$ \\
\hline om & 6.51953702 & 12.99192814 & $2.45420664 \mathrm{C}$ \\
\hline om & 6.68382946 & 15.78257974 & $7.03204051 \mathrm{C}$ \\
\hline om & 2.27816078 & 27.37108983 & $2.13178883 \mathrm{C}$ \\
\hline om & 2.12047782 & 1.40698459 & $6.70949938 \mathrm{C}$ \\
\hline om & 8.23498648 & 0.41533483 & $3.75418339 \mathrm{C}$ \\
\hline atom & 4.96835941 & 28.35914066 & $8.33209885 \mathrm{C}$ \\
\hline $\mathrm{n}$ & 0.56871711 & 14.81200255 & $0.83201643 \mathrm{C}$ \\
\hline 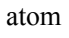 & 3.82990585 & 13.96608883 & $5.40982136 \mathrm{C}$ \\
\hline om & 8.49575886 & 13.02271248 & $3.83769104 \mathrm{C}$ \\
\hline om & 4.70760608 & 15.75175895 & $8.41551961 \mathrm{C}$ \\
\hline om & 0.30008700 & 27.40955027 & $0.75104066 \mathrm{C}$ \\
\hline atom & 4.09854329 & 1.36856241 & $5.32874170 \mathrm{C}$ \\
\hline $\mathrm{m}$ & 8.20597658 & 1.74779030 & $3.35326132 \mathrm{C}$ \\
\hline 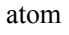 & 4.99737567 & 27.02670031 & $7.93116841 \mathrm{C}$ \\
\hline on & 0.59412542 & 16.14535877 & $1.22989915 \mathrm{C}$ \\
\hline om & 3.80449710 & 12.63271554 & $5.80768684 \mathrm{C}$ \\
\hline m & 7.89514636 & 10.86654190 & $2.68533875 \mathrm{C}$ \\
\hline om & 5.30823092 & 17.90796884 & $7.26318836 \mathrm{C}$ \\
\hline atom & 0.89732375 & 25.24939022 & $1.89666281 \mathrm{C}$ \\
\hline atom & 3.50131935 & 3.52868823 & $6.47437585 \mathrm{C}$ \\
\hline om & 7.16412068 & 3.63313471 & $1.99890962 \mathrm{C}$ \\
\hline & 6.03923629 & 25.14141238 & $6.57680402 \mathrm{C}$ \\
\hline & 1.63363877 & 18.03855932 & $2.57348159 \mathrm{C}$ \\
\hline & 2.76498980 & 10.73946359 & $7.15125166 \mathrm{C}$ \\
\hline
\end{tabular}

\section{$(S-M B A)_{2} \mathrm{~Pb}_{1-x} \mathrm{Sn}_{x} \mathbf{I}_{4}(\mathrm{x}=0.75) \quad(2 \times 2)$ unit cell, PBE+TS relaxed with "tight" default setting}

$\begin{array}{lcccc}\text { lattice_vector } & 12.72473311 & -0.00021550 & 0.06633857 \\ \text { lattice_vector } & 0.00011839 & 28.74974066 & -0.00005406 \\ \text { lattice_vector } & -0.55281439 & -0.00000162 & 12.70644499 \\ \text { atom } & 0.60201140 & 7.22340091 & 3.83540889 \mathrm{~Pb} \\ \text { atom } & 8.25079650 & 21.59780172 & 12.09756250 \mathrm{~Pb} \\ \text { atom } & 8.53071976 & 21.51871004 & 5.72819843 \mathrm{Sn} \\ \text { atom } & 1.90600336 & 21.52026285 & 11.94226561 \mathrm{Sn} \\ \text { atom } & 2.19514674 & 21.60442720 & 5.56705700 \mathrm{Sn} \\ \text { atom } & 6.65729678 & 7.22952811 & 10.36599546 \mathrm{Sn} \\ \text { atom } & 0.32211927 & 7.14393576 & 10.20439588 \mathrm{Sn} \\ \text { atom } & 6.94640929 & 7.14525977 & 3.99004484 \mathrm{Sn} \\ \text { atom } & 0.42778450 & 4.01019812 & 4.14436583 \mathrm{I} \\ \text { atom } & 6.51958968 & 4.04528759 & 10.54903019 \mathrm{I}\end{array}$




\begin{tabular}{|c|c|c|c|}
\hline atom & 8.31742866 & 24.70111994 & $5.80393096 \mathrm{I}$ \\
\hline atom & 1.67092935 & 24.69772332 & $12.10146364 \mathrm{I}$ \\
\hline tom & 2.33307188 & 18.41998974 & $5.38369277 \mathrm{I}$ \\
\hline tom & 8.42486459 & 18.38518705 & $11.78825583 \mathrm{I}$ \\
\hline atom & 0.53532293 & 10.32640430 & $10.12883745 \mathrm{I}$ \\
\hline tom & 7.18188393 & 10.32315180 & $3.83113111 \mathrm{I}$ \\
\hline atom & 0.41053959 & 10.41597436 & 4.10974731 I \\
\hline atom & 6.48178950 & 10.38743081 & $10.51758758 \mathrm{I}$ \\
\hline atom & 8.33921017 & 18.36467040 & $5.83130101 \mathrm{I}$ \\
\hline atom & 1.70614516 & 18.36617947 & $12.12279881 \mathrm{I}$ \\
\hline atom & 2.37099965 & 24.76224038 & $5.41504924 \mathrm{I}$ \\
\hline tom & 8.44212843 & 24.79081698 & $11.82300811 \mathrm{I}$ \\
\hline atom & 0.51337404 & 3.99028008 & $10.10153781 \mathrm{I}$ \\
\hline tom & 7.14645453 & 3.99128181 & $3.80985644 \mathrm{I}$ \\
\hline tom & 3.64168184 & 7.21656052 & $4.73809678 \mathrm{I}$ \\
\hline atom & 9.59926800 & 7.22646446 & $11.11940363 \mathrm{I}$ \\
\hline tom & 1.23858021 & 21.52562277 & $8.98466363 \mathrm{I}$ \\
\hline atom & 7.89440544 & 21.52864419 & $2.63442355 \mathrm{I}$ \\
\hline tom & 11.97823483 & 21.60112059 & $4.87969158 \mathrm{I}$ \\
\hline atom & 5.21056969 & 21.59129249 & $11.19459736 \mathrm{I}$ \\
\hline tom & 7.61450891 & 7.15061873 & $6.94828305 \mathrm{I}$ \\
\hline atom & 1.51110171 & 7.15392536 & $0.59184243 \mathrm{I}$ \\
\hline atom & 12.37148462 & 7.18303203 & $7.05308002 \mathrm{I}$ \\
\hline atom & 6.28721622 & 7.19105153 & $0.60806553 \mathrm{I}$ \\
\hline atom & 5.52718560 & 21.55550749 & $6.42499010 \mathrm{I}$ \\
\hline atom & 12.10684012 & 21.56006374 & $0.12493347 \mathrm{I}$ \\
\hline atom & 9.20589067 & 21.55780397 & $8.94623722 \mathrm{I}$ \\
\hline atom & 3.11822372 & 21.56582413 & $2.61791139 \mathrm{I}$ \\
\hline atom & 10.02360973 & 7.18523801 & $3.16754448 \mathrm{I}$ \\
\hline atom & 3.32529898 & 7.18079081 & $9.50783603 \mathrm{I}$ \\
\hline atom & 2.37655220 & 4.55363306 & $6.98513918 \mathrm{~N}$ \\
\hline atom & 9.00731102 & 4.55505741 & $0.69913359 \mathrm{~N}$ \\
\hline atom & 11.62732395 & 24.19610699 & $10.10228444 \mathrm{~N}$ \\
\hline atom & 5.56771117 & 24.19883085 & $3.71786031 \mathrm{~N}$ \\
\hline atom & 0.39818140 & 18.93009618 & $2.52715207 \mathrm{~N}$ \\
\hline atom & 6.47609196 & 18.92834396 & $8.94759361 \mathrm{~N}$ \\
\hline atom & 9.95005642 & 9.82137101 & $5.89673313 \mathrm{~N}$ \\
\hline atom & 3.28504469 & 9.82407553 & $12.21483385 \mathrm{~N}$ \\
\hline atom & 2.34554647 & 9.96103817 & $7.16390014 \mathrm{~N}$ \\
\hline atom & 8.97681106 & 9.95755753 & $0.86253365 \mathrm{~N}$ \\
\hline atom & 11.46049764 & 18.78859933 & $10.11837047 \mathrm{~N}$ \\
\hline atom & 5.38347486 & 18.78593366 & $3.73611668 \mathrm{~N}$ \\
\hline atom & 0.42873996 & 24.33243074 & $2.36370342 \mathrm{~N}$ \\
\hline atom & 6.50717662 & 24.33572191 & $8.76882140 \mathrm{~N}$ \\
\hline atom & 10.11687377 & 4.41385154 & $5.88067162 \mathrm{~N}$ \\
\hline atom & 3.46915669 & 4.41115093 & $12.19661431 \mathrm{~N}$ \\
\hline atom & 2.65675554 & 5.54517965 & $6.90019056 \mathrm{H}$ \\
\hline atom & 9.28823138 & 5.54773696 & $0.63375365 \mathrm{H}$ \\
\hline atom & 11.69869136 & 23.20428137 & $9.81995462 \mathrm{H}$ \\
\hline atom & 5.64929615 & 23.20626955 & $3.44128745 \mathrm{H}$ \\
\hline atom & 0.11728755 & 19.92278795 & $2.59253027 \mathrm{H}$ \\
\hline & 6.19593445 & 19.91990778 & $9.03258481 \mathrm{H}$ \\
\hline atom & 9.87877448 & 8.82953986 & $6.17907697 \mathrm{H}$ \\
\hline atom & 3.20344752 & 8.83150502 & $12.49137762 \mathrm{H}$ \\
\hline atom & 2.04246091 & 4.38457820 & $7.95678148 \mathrm{H}$ \\
\hline atom & 8.68118869 & 4.36773082 & $1.67023147 \mathrm{H}$ \\
\hline atom & 10.64359454 & 24.38907007 & $10.38423622 \mathrm{H}$ \\
\hline atom & 4.58359053 & 24.38152225 & $4.00666060 \mathrm{H}$ \\
\hline atom & 0.72429128 & 18.74276197 & $1.55604878 \mathrm{H}$ \\
\hline atom & 6.81012461 & 18.75932835 & $7.97592455 \mathrm{H}$ \\
\hline atom & 10.93374471 & 10.01439081 & $5.61467381 \mathrm{H}$ \\
\hline atom & 4.26917065 & 10.00675158 & $11.92604581 \mathrm{H}$ \\
\hline & 1.57214034 & 4.41150218 & $6.33165784 \mathrm{H}$ \\
\hline atom & 8.19696012 & 4.42728232 & $0.05042526 \mathrm{H}$ \\
\hline & 12.23859960 & 24.31582643 & $10.94231940 \mathrm{H}$ \\
\hline atom & 6.18509679 & 24.33107887 & $4.55207504 \mathrm{H}$ \\
\hline atom & 1.20853941 & 18.80228614 & $3.17584876 \mathrm{H}$ \\
\hline & 7.28052136 & 18.78611675 & $9.60104560 \mathrm{H}$ \\
\hline atom & 9.33868778 & 9.94106605 & $5.05675350 \mathrm{H}$ \\
\hline atom & 2.66768149 & 9.95637217 & $11.38060960 \mathrm{H}$ \\
\hline
\end{tabular}




\begin{tabular}{|c|c|c|c|}
\hline ator & 1.91777871 & 10.22408959 & $6.24909770 \mathrm{H}$ \\
\hline ator & 98754605 & 10.21613407 & $12.65713432 \mathrm{H}$ \\
\hline & -0.36818395 & 18.53832702 & $10.52442713 \mathrm{H}$ \\
\hline 01 & 6.27820871 & 8.53141393 & $4.20857933 \mathrm{H}$ \\
\hline & .86514588 & 24.59087408 & $3.27561270 \mathrm{H}$ \\
\hline $\mathrm{m}$ & 6.93496908 & 24.59874459 & $9.68361430 \mathrm{H}$ \\
\hline & 9.22078789 & 4.16345179 & $5.40830596 \mathrm{H}$ \\
\hline & 2.57442756 & 15668417 & $11.72413545 \mathrm{H}$ \\
\hline n & 1.60263954 & 9.99908681 & $7.90082861 \mathrm{H}$ \\
\hline & 8.24226516 & 10.00135059 & $1.60782989 \mathrm{H}$ \\
\hline & 10.68695152 & 18.73629210 & $10.82217562 \mathrm{H}$ \\
\hline & 4.60938333 & 18.73835633 & $4.44019050 \mathrm{H}$ \\
\hline & & & \\
\hline$m_{0}$ & 07920 & 791 & $12 \mathrm{H}$ \\
\hline & 248 & & $00 \mathrm{H}$ \\
\hline & 366 & & $78 \mathrm{H}$ \\
\hline & & & $7.08443889 \mathrm{H}$ \\
\hline & 9.28 & & $22 \mathrm{H}$ \\
\hline & 11.5 & 846 & $15 \mathrm{H}$ \\
\hline$n$ & 930 & 19. & $0 \mathrm{H}$ \\
\hline 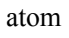 & 0.1 & 23. & $4 \mathrm{H}$ \\
\hline 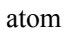 & 470 & 23. & $3 \mathrm{H}$ \\
\hline & 10.03 & & $21 \mathrm{H}$ \\
\hline & & & $21 \mathrm{H}$ \\
\hline & 2427 & $16 .($ & $87 \mathrm{H}$ \\
\hline & & & \\
\hline $\mathrm{n}$ & 10.9 & & $99 \mathrm{H}$ \\
\hline $\mathrm{m}$ & & 12. & $1 \mathrm{H}$ \\
\hline $\mathrm{m}$ & 91 & & $3 \mathrm{H}$ \\
\hline & 767 & & 8.2 \\
\hline & 10.6 & & $24 \mathrm{H}$ \\
\hline & 10 & 461 & 12. \\
\hline & & & \\
\hline 1 & & & $7 \mathrm{H}$ \\
\hline $\mathrm{m}$ & 04 & & $01 \mathrm{H}$ \\
\hline & 355 & 77 & $32 \mathrm{H}$ \\
\hline & & & \\
\hline & 06 & & 10.3 \\
\hline & 6907 & & $13 \mathrm{H}$ \\
\hline & & & $2 \mathrm{H}$ \\
\hline & & & $5 \mathrm{H}$ \\
\hline $\mathrm{m}$ & 28 & & $4 \mathrm{H}$ \\
\hline & 0574 & 936 & $39 \mathrm{H}$ \\
\hline & & & \\
\hline & 0.292 & 1000 & $302 \mathrm{H}$ \\
\hline $\mathrm{n}$ & & & $8.18847456 \mathrm{H}$ \\
\hline & 10.6 & & \\
\hline & & & 12. \\
\hline & 62 & 28. & $5 \mathrm{H}$ \\
\hline & 51 & 052 & $936 \mathrm{H}$ \\
\hline & 68 & 0.72 & $10.13491419 \mathrm{H}$ \\
\hline & 6927 & 0882 & $42 \mathrm{H}$ \\
\hline & & & \\
\hline & & & $380 \mathrm{H}$ \\
\hline & & & \\
\hline & 1.69 & 15. & $947 \mathrm{H}$ \\
\hline & 31 & & $54 \mathrm{H}$ \\
\hline & 2.82357322 & 14.90650909 & $2323 \mathrm{H}$ \\
\hline & 2.80301579 & 6986 & $5246 \mathrm{H}$ \\
\hline & 8.886 & & $266 \mathrm{H}$ \\
\hline & 12.11 & & $9770 \mathrm{H}$ \\
\hline & & 053 & $169 \mathrm{H}$ \\
\hline & -0.03342715 & 929 & $6.42356526 \mathrm{H}$ \\
\hline & & 6484 & $685 \mathrm{H}$ \\
\hline & 5.02282990 & 5.17741219 & $6.81084684 \mathrm{H}$ \\
\hline & 11.65230795 & 5.18192223 & $0.45260147 \mathrm{H}$ \\
\hline & 11.99666532 & 23.57509848 & $1946 \mathrm{H}$ \\
\hline & 5.88165906 & 23.56694352 & $1.08693657 \mathrm{H}$ \\
\hline & & 19.55679907 & $2.84007757 \mathrm{H}$ \\
\hline & 3.82978387 & 19.55222152 & $9.12194525 \mathrm{H}$ \\
\hline
\end{tabular}




\begin{tabular}{|c|c|c|c|}
\hline atom & 9.58076902 & 9.20027115 & $8.52778693 \mathrm{H}$ \\
\hline atom & 3.52384195 & 9.19212797 & $2.13930924 \mathrm{H}$ \\
\hline atom & 5.64060598 & 3.52189062 & $6.76241870 \mathrm{H}$ \\
\hline om & 12.26642021 & 3.52574512 & $0.38620372 \mathrm{H}$ \\
\hline tom & 12.10305030 & 25.23231833 & $6.86520454 \mathrm{H}$ \\
\hline om & 5.97754803 & 25.22028758 & $0.46847745 \mathrm{H}$ \\
\hline atom & 9.86381441 & 17.90060279 & $2.90641112 \mathrm{H}$ \\
\hline atom & 3.21202364 & 17.89670467 & $9.17028235 \mathrm{H}$ \\
\hline atom & 9.47440299 & 10.85746288 & $9.13386098 \mathrm{H}$ \\
\hline atom & 3.42793514 & 10.84545568 & $2.75777880 \mathrm{H}$ \\
\hline atom & 11.51184202 & 4.12492201 & $1.87587379 \mathrm{H}$ \\
\hline atom & 4.84530406 & 4.12191579 & $8.23005678 \mathrm{H}$ \\
\hline atom & 10.57474823 & 24.63932620 & $7.54460719 \mathrm{H}$ \\
\hline atom & 4.46295100 & 24.63274772 & $1.18146117 \mathrm{H}$ \\
\hline atom & 4.00733709 & 18.49681574 & $7.70268805 \mathrm{H}$ \\
\hline atom & 10.61837955 & 18.49986131 & $1.41677556 \mathrm{H}$ \\
\hline atom & 11.00268661 & 10.26446104 & $8.45440333 \mathrm{H}$ \\
\hline atom & 4.94255212 & 10.25790602 & $2.04482556 \mathrm{H}$ \\
\hline atom & 5.04245851 & 9.53524314 & $6.80235289 \mathrm{H}$ \\
\hline atom & 11.66570456 & 9.52603994 & $0.45942120 \mathrm{H}$ \\
\hline atom & 11.97872251 & 19.22354461 & $7.45066122 \mathrm{H}$ \\
\hline atom & 5.88848547 & 19.21985114 & $1.06447046 \mathrm{H}$ \\
\hline atom & 10.46459499 & 23.90104921 & $2.83309515 \mathrm{H}$ \\
\hline atom & 3.81028824 & 23.91007667 & $9.13043603 \mathrm{H}$ \\
\hline atom & 9.59869446 & 4.84872184 & $8.54838308 \mathrm{H}$ \\
\hline atom & 3.51691202 & 4.84501336 & $2.16180119 \mathrm{H}$ \\
\hline atom & 5.52480928 & 11.22822720 & $6.99561756 \mathrm{H}$ \\
\hline atom & 12.15523214 & 11.21818305 & $0.64216827 \mathrm{H}$ \\
\hline atom & 11.82768214 & 17.53080534 & $6.95280496 \mathrm{H}$ \\
\hline atom & 5.73265528 & 17.52701108 & $0.56869228 \mathrm{H}$ \\
\hline atom & 9.97523663 & 25.59327107 & $2.65041134 \mathrm{H}$ \\
\hline atom & 3.32795323 & 25.60305804 & $8.93708365 \mathrm{H}$ \\
\hline atom & 9.74973086 & 3.15596845 & $9.04621234 \mathrm{H}$ \\
\hline atom & 3.67276901 & 3.15217302 & $2.65758899 \mathrm{H}$ \\
\hline atom & 4.48750974 & 10.75817086 & $5.63795030 \mathrm{H}$ \\
\hline atom & 10.54299500 & 10.74811989 & $12.00799878 \mathrm{H}$ \\
\hline atom & 0.39214455 & 18.00742065 & $8.00498615 \mathrm{H}$ \\
\hline atom & 7.02838183 & 18.00208673 & $1.68011081 \mathrm{H}$ \\
\hline atom & 11.03467631 & 25.12303118 & $3.99096279 \mathrm{H}$ \\
\hline atom & 4.36524314 & 25.13302447 & $10.29478427 \mathrm{H}$ \\
\hline atom & 8.46051965 & 3.63243367 & $7.92771479 \mathrm{H}$ \\
\hline atom & 2.37702809 & 3.62726170 & $1.54616983 \mathrm{H}$ \\
\hline atom & 2.74821089 & 1.67499906 & $4.89357469 \mathrm{H}$ \\
\hline atom & 8.81881116 & 1.67471329 & $11.28887254 \mathrm{H}$ \\
\hline atom & 1.04695016 & 27.07416661 & $9.77161567 \mathrm{H}$ \\
\hline atom & 7.69230554 & 27.06871288 & $3.44812563 \mathrm{H}$ \\
\hline atom & 0.03382495 & 16.04969231 & 4.64387002 H \\
\hline atom & 6.10437019 & 16.04968912 & $11.03912982 \mathrm{H}$ \\
\hline atom & 7.80576165 & 12.69925304 & $6.16103765 \mathrm{H}$ \\
\hline atom & 1.16044145 & 12.69401029 & $12.48464761 \mathrm{H}$ \\
\hline atom & 2.70655077 & 12.45445085 & $5.47580812 \mathrm{H}$ \\
\hline atom & 8.78646856 & 12.45323588 & $11.86583056 \mathrm{H}$ \\
\hline atom & 0.46182538 & 16.29629331 & $9.76441978 \mathrm{H}$ \\
\hline atom & 7.10198998 & 16.29512757 & $3.45223970 \mathrm{H}$ \\
\hline atom & 0.06640156 & 26.82812327 & $4.06681882 \mathrm{H}$ \\
\hline atom & 6.14629446 & 26.82918737 & $10.45692464 \mathrm{H}$ \\
\hline atom & 8.39079224 & 1.92129114 & $6.16833372 \mathrm{H}$ \\
\hline atom & 1.75061398 & 1.92033700 & $12.48047986 \mathrm{H}$ \\
\hline atom & 9.72974678 & 0.08391055 & $3.26674319 \mathrm{H}$ \\
\hline atom & 3.07446865 & 0.07356233 & $9.57615600 \mathrm{H}$ \\
\hline atom & 9.11269908 & 28.67802907 & $9.27113628 \mathrm{H}$ \\
\hline atom & 3.03709218 & 28.68411730 & $2.88240209 \mathrm{H}$ \\
\hline atom & 5.77822310 & 14.44843854 & $6.35650593 \mathrm{H}$ \\
\hline atom & 12.40039099 & 14.45888383 & $0.02584719 \mathrm{H}$ \\
\hline atom & 12.46477464 & 14.30313891 & $6.72788660 \mathrm{H}$ \\
\hline tom & 6.36850310 & 14.30924389 & $0.34381053 \mathrm{H}$ \\
\hline atom & 10.01954423 & 12.49606846 & $3.41771815 \mathrm{H}$ \\
\hline & 3.37424691 & 12.50396571 & $9.73610097 \mathrm{H}$ \\
\hline atom & 8.96317227 & 16.24199908 & $8.96037473 \mathrm{H}$ \\
\hline atom & 2.87931984 & 16.24035043 & $2.57742214 \mathrm{H}$ \\
\hline
\end{tabular}




\begin{tabular}{|c|c|c|c|}
\hline atom & 5.47856015 & 26.87873248 & $6.19663413 \mathrm{H}$ \\
\hline atom & 12.11101003 & 26.87081307 & $-0.12514049 \mathrm{H}$ \\
\hline tom & 12.61420868 & 1.86717382 & $7.03857817 \mathrm{H}$ \\
\hline om & 6.52609477 & 1.86549807 & $0.64887464 \mathrm{H}$ \\
\hline tom & 3.55596342 & 2.42309058 & $9.05671329 \mathrm{H}$ \\
\hline om & 10.19835903 & 2.43488430 & $2.73990870 \mathrm{H}$ \\
\hline atom & 9.65305170 & 26.32585644 & $8.82645649 \mathrm{H}$ \\
\hline atom & 3.57237132 & 26.33192233 & $2.43326608 \mathrm{H}$ \\
\hline tom & 11.93182352 & 16.80985760 & $0.55275915 \mathrm{H}$ \\
\hline atom & 5.29665718 & 16.79793853 & $6.87599720 \mathrm{H}$ \\
\hline atom & 11.92435229 & 11.95098677 & $7.17261822 \mathrm{H}$ \\
\hline atom & 5.83320457 & 11.95706277 & $0.79295957 \mathrm{H}$ \\
\hline atom & 3.72436178 & 10.53909126 & $8.61154973 \mathrm{H}$ \\
\hline atom & 10.37861617 & 10.53683316 & $2.28725555 \mathrm{H}$ \\
\hline atom & 10.10148946 & 18.20460875 & $8.65487773 \mathrm{H}$ \\
\hline atom & 4.01638346 & 18.20446912 & $2.27925912 \mathrm{H}$ \\
\hline atom & 11.75179946 & 24.91171434 & $1.00526794 \mathrm{H}$ \\
\hline atom & 5.12838419 & 24.91390734 & $7.32118493 \mathrm{H}$ \\
\hline atom & 11.47589650 & 3.82976080 & $7.34412757 \mathrm{H}$ \\
\hline atom & 5.38902737 & 3.82960445 & $0.94704486 \mathrm{H}$ \\
\hline atom & 3.53447671 & 3.73197885 & $5.47047885 \mathrm{H}$ \\
\hline atom & 9.57375009 & 3.74102292 & $11.85864460 \mathrm{H}$ \\
\hline atom & 0.50500860 & 25.00791954 & $8.99540523 \mathrm{H}$ \\
\hline atom & 7.14795681 & 25.01032782 & $2.64294388 \mathrm{H}$ \\
\hline atom & 12.00367284 & 18.11589831 & $4.14044567 \mathrm{H}$ \\
\hline atom & 5.31815704 & 18.10671139 & $10.46225192 \mathrm{H}$ \\
\hline atom & 8.34766162 & 10.63298410 & $6.93733648 \mathrm{H}$ \\
\hline atom & 2.25758930 & 10.63558229 & $0.58328646 \mathrm{H}$ \\
\hline atom & 2.42933079 & 15.03203641 & $7.67112414 \mathrm{C}$ \\
\hline atom & 9.06639041 & 15.02683881 & $1.36014097 \mathrm{C}$ \\
\hline atom & 10.98297584 & 13.71597086 & $10.00287427 \mathrm{C}$ \\
\hline atom & 4.89918410 & 13.71409567 & $3.61926682 \mathrm{C}$ \\
\hline atom & 0.33887516 & 0.65213732 & $1.86623331 \mathrm{C}$ \\
\hline atom & 6.42331502 & 0.65704597 & $8.26163547 \mathrm{C}$ \\
\hline atom & 10.59450901 & 28.09088669 & $5.99620943 \mathrm{C}$ \\
\hline atom & 3.95356287 & 28.08901553 & $12.31343586 \mathrm{C}$ \\
\hline atom & 2.40855428 & 14.33869003 & $6.46253191 \mathrm{C}$ \\
\hline atom & 9.04261617 & 14.33564273 & $0.15028866 \mathrm{C}$ \\
\hline atom & 12.18865777 & 14.41047472 & $10.08054068 \mathrm{C}$ \\
\hline atom & 6.10440109 & 14.40904903 & $3.69967848 \mathrm{C}$ \\
\hline atom & 0.36280439 & 28.71063033 & $3.07601309 \mathrm{C}$ \\
\hline atom & 6.44424699 & 28.71342726 & $9.47017669 \mathrm{C}$ \\
\hline atom & 9.38870413 & 0.03566837 & $5.91859426 \mathrm{C}$ \\
\hline atom & 2.74822428 & 0.03424641 & $12.23305313 \mathrm{C}$ \\
\hline atom & 2.64534111 & 28.28281188 & $7.52833741 \mathrm{C}$ \\
\hline atom & 9.29324554 & 28.28718994 & $1.22209171 \mathrm{C}$ \\
\hline atom & 11.13736090 & 0.46951257 & $9.79762471 \mathrm{C}$ \\
\hline atom & 5.06251220 & 0.46974396 & $3.41235105 \mathrm{C}$ \\
\hline atom & 0.11229506 & 13.91253734 & $2.00408905 \mathrm{C}$ \\
\hline atom & 6.20744833 & 13.90786718 & $8.40426530 \mathrm{C}$ \\
\hline atom & 10.44000856 & 14.84444984 & $6.20143079 \mathrm{C}$ \\
\hline atom & 3.79014398 & 14.84469246 & $12.52042390 \mathrm{C}$ \\
\hline atom & 2.57154039 & -0.02204621 & $6.21174840 \mathrm{C}$ \\
\hline atom & 8.65758049 & -0.02080331 & $12.61143099 \mathrm{C}$ \\
\hline atom & -0.27627337 & 28.77395738 & $9.87186710 \mathrm{C}$ \\
\hline atom & 6.37240830 & 28.77063964 & $3.55345951 \mathrm{C}$ \\
\hline atom & 0.19504354 & 14.35422839 & $3.32126014 \mathrm{C}$ \\
\hline atom & 6.28108869 & 14.35269468 & $9.72092145 \mathrm{C}$ \\
\hline atom & 9.12902061 & 14.39899598 & $6.06077967 \mathrm{C}$ \\
\hline atom & 2.48035610 & 14.39589818 & $12.37929022 \mathrm{C}$ \\
\hline atom & 2.76840349 & 14.36675907 & $8.84603172 \mathrm{C}$ \\
\hline atom & 9.40889618 & 14.35983822 & $2.53290526 \mathrm{C}$ \\
\hline atom & 9.82483149 & 14.38028722 & $9.60882376 \mathrm{C}$ \\
\hline atom & 3.74116559 & 14.37808262 & $3.22414963 \mathrm{C}$ \\
\hline atom & -0.00334831 & 28.73481035 & $0.69340148 \mathrm{C}$ \\
\hline atom & 6.08436254 & 28.74151845 & $7.08668382 \mathrm{C}$ \\
\hline atom & 11.75253897 & 0.00546193 & $6.39025298 \mathrm{C}$ \\
\hline & 5.11145393 & 0.00324616 & $12.70861031 \mathrm{C}$ \\
\hline atom & 4.82355859 & 4.14929677 & $7.13534112 \mathrm{C}$ \\
\hline atom & 11.46063229 & 4.15382169 & $0.78208891 \mathrm{C}$ \\
\hline
\end{tabular}




\begin{tabular}{|c|c|c|c|}
\hline & 11.66456598 & 24.60480184 & $7.64907298 \mathrm{C}$ \\
\hline atom & 5.55454218 & 24.59821253 & $1.26497409 \mathrm{C}$ \\
\hline atum & 10.66958415 & 18.52871430 & $2.51056168 \mathrm{C}$ \\
\hline & 4.02907345 & 18.52412477 & $8.79740448 \mathrm{C}$ \\
\hline & 9.91286737 & 10.22997574 & $8.34995874 \mathrm{C}$ \\
\hline & 3.85096523 & 10.22339259 & $1.96128557 \mathrm{C}$ \\
\hline on & 4.70074104 & 10.57262858 & $6.69759832 \mathrm{C}$ \\
\hline & 11.32484651 & 10.56416062 & $0.35822372 \mathrm{C}$ \\
\hline & 12.07059056 & 18.18582270 & $7.79528397 \mathrm{C}$ \\
\hline 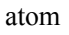 & 5.98098607 & 18.18223988 & $1.40938583 \mathrm{C}$ \\
\hline $\mathrm{m}$ & 10.80555268 & 24.93912207 & $2.93430720 \mathrm{C}$ \\
\hline & 4.15201939 & 24.94745304 & $9.23514437 \mathrm{C}$ \\
\hline & 9.50681090 & 3.81101281 & $8.20374701 \mathrm{C}$ \\
\hline & 3.42442195 & 3.80740673 & $1.81689096 \mathrm{C}$ \\
\hline & 2.82095557 & 1.31599719 & $5.91922068 \mathrm{C}$ \\
\hline & 8.89930639 & 1.31766415 & $12.31467382 \mathrm{C}$ \\
\hline $\mathrm{m}$ & 0.02685517 & 27.43417083 & $9.64495073 \mathrm{C}$ \\
\hline 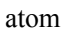 & 6.67283357 & 27.4 & $173 \mathrm{C}$ \\
\hline $\mathrm{m}$ & -0.04 & 15.6 & $55 \mathrm{C}$ \\
\hline & 6.03 & 15.6 & 10.0 \\
\hline & 8.82585770 & 13.05 & $6.28772021 \mathrm{C}$ \\
\hline & 2.17991937 & 13.0 & $12.61024534 \mathrm{C}$ \\
\hline & 3.17686609 & & $6.93007622 \mathrm{C}$ \\
\hline $\mathrm{n}$ & $9.80 \mathrm{~s}$ & & 0.61 \\
\hline $\mathrm{m}$ & 11.75 & & $56 \mathrm{C}$ \\
\hline $\mathrm{m}$ & 5.67 & 26.5 & $1 \mathrm{C}$ \\
\hline $\mathrm{m}$ & 12.32095406 & 16. & $504 \mathrm{C}$ \\
\hline & 5.67574303 & 16.5 & $347 \mathrm{C}$ \\
\hline & 9.81 & 12.1 & $34 \mathrm{C}$ \\
\hline & 3.72 & 68 & $902 \mathrm{C}$ \\
\hline & & 12. & $05 \mathrm{C}$ \\
\hline $\mathrm{m}$ & 9.70 & 12.2 & $7 \mathrm{C}$ \\
\hline $\mathrm{m}$ & $11.0^{\circ}$ & & $80 \mathrm{C}$ \\
\hline $\mathrm{m}$ & 4.98 & 16.4 & $7 \mathrm{C}$ \\
\hline & 12.42707160 & 26.67397631 & $2.00275708 \mathrm{C}$ \\
\hline & 5.78864554 & 26.67828110 & $8.32520229 \mathrm{C}$ \\
\hline & 10.50316807 & & \\
\hline & 4.4 & & 0.2 \\
\hline m & 2.71 & 12.9 & 6.4 \\
\hline & 9.354 & 12.9 & $25 \mathrm{C}$ \\
\hline & 12.234 & 50070 & $9.77158645 \mathrm{C}$ \\
\hline & 6.14958720 & 15.76736312 & $3.39188136 \mathrm{C}$ \\
\hline & 0.05080320 & 27.3 & $3.11118069 \mathrm{C}$ \\
\hline & & & $9.50204756 \mathrm{C}$ \\
\hline & & & \\
\hline & 2.70 & 1.3 & 12.5 \\
\hline & 2.98 & 0.42 & $40 \mathrm{C}$ \\
\hline & 9.62975258 & 0.42869609 & $2.23942775 \mathrm{C}$ \\
\hline & 10.13363844 & 28.33055165 & $9.41665926 \mathrm{C}$ \\
\hline & 4.05713277 & 28.33403988 & $3.02830700 \mathrm{C}$ \\
\hline & 12.50040312 & & $17020 \mathrm{C}$ \\
\hline & 5.87 & 14.7 & 7.38 \\
\hline & 11.44382469 & 13.9 & $790 \mathrm{C}$ \\
\hline & 5.34845224 & 13.95920682 & $0.19793982 \mathrm{C}$ \\
\hline & 3.08293192 & 13.01011824 & $8.81563235 \mathrm{C}$ \\
\hline & 9.72465829 & 13.00348028 & $2.49912023 \mathrm{C}$ \\
\hline & 9.86911298 & 15.73684222 & $9.29566925 \mathrm{C}$ \\
\hline & 3.78513938 & 15.73503904 & $2.91273942 \mathrm{C}$ \\
\hline & 12.40 & 27.3 & $130 \mathrm{C}$ \\
\hline & 5.76985881 & 27.38487907 & $7.11709887 \mathrm{C}$ \\
\hline & 11.70825853 & 1.36202965 & $3030 \mathrm{C}$ \\
\hline & 5.62028186 & 1.36020287 & $0.31355260 \mathrm{C}$ \\
\hline & 3.25293402 & 1.75303053 & $8.25190746 \mathrm{C}$ \\
\hline & 9.89432517 & 1.76148529 & $1.93808451 \mathrm{C}$ \\
\hline & 10.44259232 & 26.99683154 & $9.16598366 \mathrm{C}$ \\
\hline & 4.36323581 & 27.00028534 & $2.77448300 \mathrm{C}$ \\
\hline & & & $1.35455288 \mathrm{C}$ \\
\hline & 5.59969403 & 16.12785054 & $7.68079286 \mathrm{C}$ \\
\hline & 11.13483507 & 12.62200512 & $6.83308068 \mathrm{C}$ \\
\hline & 5.04233826 & 12.62545604 & $0.45176233 \mathrm{C}$ \\
\hline
\end{tabular}




$\begin{array}{lccc}\text { atom } & 3.49662867 & 10.85337027 & 7.58410363 \mathrm{C} \\ \text { atom } & 10.13500445 & 10.84895402 & 1.26278167 \mathrm{C} \\ \text { atom } & 11.11528438 & 17.89585610 & 8.94338315 \mathrm{C} \\ \text { atom } & 5.03120746 & 17.89419846 & 2.56241108 \mathrm{C} \\ \text { atom } & 11.99541423 & 25.22377960 & 2.02975417 \mathrm{C} \\ \text { atom } & 5.35613583 & 25.22814295 & 8.34863238 \mathrm{C} \\ \text { atom } & 10.46209374 & 3.52105407 & 7.05562654 \mathrm{C} \\ \text { atom } & 4.37420548 & 3.51937326 & 0.66386820 \mathrm{C} \\ \text { atom } & 3.51296759 & 3.64028034 & 6.56533546 \mathrm{C} \\ \text { atom } & 10.13476057 & 3.64619823 & 0.24690245 \mathrm{C} \\ \text { atom } & 12.13710864 & 25.10680731 & 9.00066473 \mathrm{C} \\ \text { atom } & 6.05400348 & 25.10699818 & 2.60423919 \mathrm{C} \\ \text { atom } & 11.99545725 & 18.02106021 & 3.04574729 \mathrm{C} \\ \text { atom } & 5.33965556 & 18.01504086 & 9.36739557 \mathrm{C} \\ \text { atom } & 9.44029961 & 10.73204492 & 6.99838441 \mathrm{C} \\ \text { atom } & 3.35154279 & 10.73222485 & 0.62201315 \mathrm{C}\end{array}$

\section{$(S \text {-MBA })_{2} \mathrm{~Pb}_{1-x} \mathrm{Sn}_{x} \mathbf{I}_{4}(\mathrm{x}=1) \quad \mathbf{c}(2 \times 2)$ unit cell, $\mathrm{PBE}+\mathrm{TS}$ relaxed with "tight" default setting}

\begin{tabular}{|c|c|c|c|c|c|c|}
\hline \multirow{2}{*}{\multicolumn{2}{|c|}{$\begin{array}{l}\text { lattice_vector } \\
\text { lattice_vector }\end{array}$}} & \multicolumn{2}{|c|}{8.83815684} & \multicolumn{2}{|c|}{0.00048798} & 0.00000464 \\
\hline & & -0.00 & 76591 & $28.71^{\prime}$ & 19070 & 0.00069719 \\
\hline lattice & vector & -0.00 & 0189 & -0.000 & 7264 & 9.17820467 \\
\hline atom & 3.2821 & 3405 & 7.220 & 2460 & 2.332 & $1351 \mathrm{Sn}$ \\
\hline atom & 1.1351 & 1295 & 21.49 & 97995 & 6.921 & $8999 \mathrm{Sn}$ \\
\hline atom & 5.5540 & 7035 & 21.578 & 77153 & 2.257 & $27320 \mathrm{Sn}$ \\
\hline atom & 7.7012 & 28279 & 7.138 & 94650 & 6.845 & $7435 \mathrm{Sn}$ \\
\hline atom & 3.3086 & 55672 & 4.035 & 03165 & 2.564 & 4932 I \\
\hline atom & 1.1086 & 54204 & 24.68 & 31837 & 7.153 & 70312 I \\
\hline atom & 5.5281 & 2371 & 18.39 & 55955 & 2.025 & 59999 I \\
\hline atom & 7.7273 & 30544 & 10.32 & 13417 & 6.614 & $51071 \mathrm{I}$ \\
\hline atom & 3.2695 & $\$ 9391$ & 10.37 & 68155 & 2.554 & $4760 \mathrm{I}$ \\
\hline atom & 1.1476 & 58077 & 18.33 & 59233 & 7.143 & 14725 I \\
\hline atom & 5.566 & +1106 & 24.73 & 30174 & 2.035 & 30936 I \\
\hline atom & 7.6890 & 3488 & 3.981 & 50055 & 6.624 & $1725 \mathrm{I}$ \\
\hline atom & 5.8632 & 3791 & 7.215 & 16711 & 0.732 & 5192 I \\
\hline atom & 7.3922 & 25257 & 21.502 & 79836 & 5.321 & 66186 I \\
\hline atom & 2.9732 & 2443 & 21.573 & 38903 & 3.857 & $60525 \mathrm{I}$ \\
\hline atom & 1.4441 & 1345 & 7.144 & 05348 & 8.446 & 8272 I \\
\hline atom & $4.773 \mathrm{~S}$ & 5926 & 7.185 & 55101 & 5.031 & 3587 I \\
\hline atom & 8.4813 & 39916 & 21.532 & 39752 & 0.442 & 1777 I \\
\hline atom & 4.0624 & 12158 & 21.54 & 88855 & 8.736 & $10025 \mathrm{I}$ \\
\hline atom & 0.3548 & 36197 & 7.173 & 47314 & 4.147 & 0622 I \\
\hline atom & 6.7115 & 5961 & 4.540 & 30764 & 3.134 & $5005 \mathrm{~N}$ \\
\hline atom & 6.5438 & 39069 & 24.17 & 54698 & 7.724 & $53809 \mathrm{~N}$ \\
\hline atom & 2.1251 & 3289 & 18.89 & 57943 & 1.454 & $62693 \mathrm{~N}$ \\
\hline atom & 2.2921 & 4171 & 9.818 & 80355 & 6.043 & $1954 \mathrm{~N}$ \\
\hline atom & 6.8168 & 35159 & 9.955 & 44742 & 3.264 & $5661 \mathrm{~N}$ \\
\hline atom & 6.4385 & $\$ 9499$ & 18.76 & 35252 & 7.854 & $23032 \mathrm{~N}$ \\
\hline atom & 2.0191 & 8591 & 24.313 & 73931 & 1.325 & $10099 \mathrm{~N}$ \\
\hline atom & 2.3981 & 0144 & 4.403 & 65536 & 5.913 & $8028 \mathrm{~N}$ \\
\hline atom & 6.8452 & 1767 & 5.534 & 14002 & 2.883 & $4308 \mathrm{H}$ \\
\hline atom & 6.4102 & 2314 & 23.18 & 71470 & 7.473 & $99517 \mathrm{H}$ \\
\hline atom & 1.9913 & 34079 & 19.89 & 40249 & 1.706 & $16297 \mathrm{H}$ \\
\hline atom & 2.4259 & 1017 & 8.824 & 97716 & 6.295 & $0961 \mathrm{H}$ \\
\hline atom & 7.1877 & 79403 & 4.361 & 09590 & 4.043 & $6874 \mathrm{H}$ \\
\hline atom & 6.0676 & 56256 & 24.35 & 76993 & 8.633 & $26953 \mathrm{H}$ \\
\hline atom & 1.6489 & 3778 & $18.71 \mathrm{~S}$ & 29398 & 0.545 & $99403 \mathrm{H}$ \\
\hline atom & 2.7683 & 35468 & 9.998 & 08147 & 5.134 & $0481 \mathrm{H}$ \\
\hline atom & 5.6836 & 52438 & 4.400 & 57916 & 3.268 & $4248 \mathrm{H}$ \\
\hline atom & 7.5718 & 32718 & 24.317 & 26725 & 7.858 & $62751 \mathrm{H}$ \\
\hline atom & 3.1530 & 8531 & 18.75 & 96695 & 1.320 & $64897 \mathrm{H}$ \\
\hline atom & 1.2641 & 9089 & 9.958 & 42673 & 5.909 & $3015 \mathrm{H}$ \\
\hline atom & 5.8579 & 1439 & $10.21 \mathrm{~S}$ & 36386 & 2.949 & $37663 \mathrm{H}$ \\
\hline atom & 7.3975 & 4535 & 18.498 & 46367 & 7.538 & $75550 \mathrm{H}$ \\
\hline atom & 2.9781 & 0062 & 24.57 & 73484 & 1.640 & $58591 \mathrm{H}$ \\
\hline atom & 1.4391 & 9849 & 4.139 & 1887 & 6.229 & $5315 \mathrm{H}$ \\
\hline atom & 6.8471 & 17536 & 9.998 & 85025 & 4.310 & $0716 \mathrm{H}$ \\
\hline
\end{tabular}




\begin{tabular}{|c|c|c|c|}
\hline atom & 6.40824747 & 18.71891869 & $8.90017913 \mathrm{H}$ \\
\hline atom & 1.98885120 & 24.35714361 & $0.27914935 \mathrm{H}$ \\
\hline atom & 2.42842929 & 4.36027600 & $4.86772682 \mathrm{H}$ \\
\hline atom & 6.96573073 & 8.97050146 & $2.99054265 \mathrm{H}$ \\
\hline atom & 6.28971191 & 19.74729968 & $7.57997409 \mathrm{H}$ \\
\hline atom & 1.87042734 & 23.32877907 & $1.59937427 \mathrm{H}$ \\
\hline atom & 2.54684221 & 5.38861328 & $6.18802212 \mathrm{H}$ \\
\hline atom & 7.10275565 & 16.08871432 & $3.70175350 \mathrm{H}$ \\
\hline atom & 6.15274198 & 12.62907207 & $8.29076624 \mathrm{H}$ \\
\hline atom & 1.73436428 & 1.72979405 & $0.88783603 \mathrm{H}$ \\
\hline atom & 2.68300193 & 26.98758829 & $5.47760184 \mathrm{H}$ \\
\hline atom & 5.53374908 & 14.86059932 & $2.24337278 \mathrm{H}$ \\
\hline atom & 7.72172578 & 13.85719843 & $6.83246050 \mathrm{H}$ \\
\hline atom & 3.30349204 & 0.50183552 & $2.34613873 \mathrm{H}$ \\
\hline atom & 1.11385822 & 28.21555906 & $6.93594266 \mathrm{H}$ \\
\hline atom & 7.32271201 & 27.19048194 & $3.57144358 \mathrm{H}$ \\
\hline atom & 5.93278921 & 1.52740198 & $8.15987116 \mathrm{H}$ \\
\hline atom & 1.51298507 & 12.83149356 & $1.01865619 \mathrm{H}$ \\
\hline atom & 2.90431126 & 15.88588342 & $5.60782742 \mathrm{H}$ \\
\hline atom & 5.54938330 & 27.98179729 & $2.05491885 \mathrm{H}$ \\
\hline atom & 7.70609090 & 0.73604564 & $6.64330205 \mathrm{H}$ \\
\hline atom & 3.28619769 & 13.62303307 & $2.53522188 \mathrm{H}$ \\
\hline atom & 1.13110742 & 15.09435190 & $7.12435354 \mathrm{H}$ \\
\hline atom & 0.21696355 & 14.89536155 & $4.70736045 \mathrm{H}$ \\
\hline atom & 4.20036507 & 13.82200610 & $0.11825581 \mathrm{H}$ \\
\hline atom & 8.62027728 & 0.53715382 & $9.06035561 \mathrm{H}$ \\
\hline atom & 4.63522262 & 28.18065401 & $4.47193575 \mathrm{H}$ \\
\hline atom & 8.38390667 & 5.17996129 & $1.07776740 \mathrm{H}$ \\
\hline atom & 4.87152608 & 23.53788562 & $5.66744494 \mathrm{H}$ \\
\hline atom & 0.45269369 & 19.53808064 & $3.51181663 \mathrm{H}$ \\
\hline atom & 3.96455441 & 9.17925670 & $8.10066721 \mathrm{H}$ \\
\hline atom & 8.77217264 & 3.52759126 & $0.58477684 \mathrm{H}$ \\
\hline atom & 4.48326779 & 25.19025435 & $5.17451123 \mathrm{H}$ \\
\hline atom & 0.06463971 & 17.88567460 & $4.00477253 \mathrm{H}$ \\
\hline atom & 4.35262144 & 10.83164590 & $8.59374390 \mathrm{H}$ \\
\hline atom & 0.46885456 & 4.12177222 & $2.16843384 \mathrm{H}$ \\
\hline atom & 3.94844786 & 24.59561461 & $6.75816202 \mathrm{H}$ \\
\hline atom & 8.36788759 & 18.48072974 & $2.42112572 \mathrm{H}$ \\
\hline atom & 4.88754559 & 10.23710614 & $7.01005857 \mathrm{H}$ \\
\hline atom & 8.39332216 & 9.51341308 & $1.05028268 \mathrm{H}$ \\
\hline atom & 4.86219086 & 19.20438361 & $5.63962412 \mathrm{H}$ \\
\hline atom & 0.44280820 & 23.87152684 & $3.53965658 \mathrm{H}$ \\
\hline atom & 3.97456400 & 4.84587624 & $8.12821300 \mathrm{H}$ \\
\hline atom & 8.86877880 & 11.20357304 & $0.82068647 \mathrm{H}$ \\
\hline atom & 4.38678381 & 17.51421371 & $5.40991320 \mathrm{H}$ \\
\hline atom & -0.03286770 & 25.56163101 & $3.76935448 \mathrm{H}$ \\
\hline atom & 4.45026741 & 3.15577357 & $8.35779017 \mathrm{H}$ \\
\hline atom & 7.16719905 & 10.73770629 & $0.65344622 \mathrm{H}$ \\
\hline atom & 6.08835762 & 17.98013003 & $5.24277253 \mathrm{H}$ \\
\hline atom & 1.66877176 & 25.09595067 & $3.93655199 \mathrm{H}$ \\
\hline atom & 2.74862980 & 3.62142515 & $8.52509065 \mathrm{H}$ \\
\hline atom & 5.44651636 & 1.66843459 & $1.41016099 \mathrm{H}$ \\
\hline atom & 7.80894434 & 27.04939546 & $5.99988643 \mathrm{H}$ \\
\hline atom & 3.39052534 & 16.02688653 & $3.17934576 \mathrm{H}$ \\
\hline atom & 1.02676750 & 12.69049945 & $7.76837597 \mathrm{H}$ \\
\hline atom & 5.84320077 & 12.44529991 & $1.82476150 \mathrm{H}$ \\
\hline atom & 7.41228222 & 16.27250397 & $6.41398059 \mathrm{H}$ \\
\hline atom & 2.99256662 & 26.80368972 & $2.76532402 \mathrm{H}$ \\
\hline atom & 1.42476753 & 1.91371378 & $7.35380301 \mathrm{H}$ \\
\hline atom & 0.23767782 & 0.06455424 & $4.38637888 \mathrm{H}$ \\
\hline atom & 4.17967024 & 28.65286139 & $8.97619415 \mathrm{H}$ \\
\hline atom & 8.59957814 & 14.42352697 & $0.20306633 \mathrm{H}$ \\
\hline atom & 4.65588916 & 14.29434178 & $4.79218270 \mathrm{H}$ \\
\hline atom & 0.55547429 & 12.49210152 & $4.27246968 \mathrm{H}$ \\
\hline atom & 3.86185385 & 16.22518416 & $8.86169375 \mathrm{H}$ \\
\hline atom & 8.28028563 & 26.85112030 & $0.31762041 \mathrm{H}$ \\
\hline atom & 4.97518288 & 1.86677240 & $4.90606410 \mathrm{H}$ \\
\hline atom & 9.02551086 & 2.41659515 & $3.68540384 \mathrm{H}$ \\
\hline atom & 4.22998663 & 26.30129082 & $8.27517219 \mathrm{H}$ \\
\hline atom & -0.18854995 & 16.77461117 & $0.90410687 \mathrm{H}$ \\
\hline
\end{tabular}




\begin{tabular}{|c|c|c|c|}
\hline atom & 4.60586283 & 11.94277321 & \\
\hline om & 8.81756501 & 10.52802988 & $3.24311110 \mathrm{H}$ \\
\hline & .43788524 & 18.18974484 & \\
\hline $\mathrm{om}$ & 0.01840814 & 24.88613501 & 1.34688974 \\
\hline $\mathrm{om}$ & 4.39888442 & 3.83127771 & $5.93536853 \mathrm{H}$ \\
\hline om & 6.39301474 & 3.72940572 & $1.25090927 \mathrm{H}$ \\
\hline & 6.86243907 & 24.98840910 & \\
\hline om & 2.44377289 & 18.08775083 & \\
\hline & 1.97350080 & 10.62962933 & 7.92757 \\
\hline om & 7.24780920 & 15.02244955 & \\
\hline & 6.00767052 & 13.69533683 & 8.124 \\
\hline om & 1.589 & 0.66351551 & \\
\hline om & 2.82793091 & 28.05387226 & 5.6438 \\
\hline & 6.35 & 14.32975112 & \\
\hline $\mathrm{om}$ & 6.89943045 & 14.38804559 & 7.30 \\
\hline & 2.47 & 28.68808891 & \\
\hline om & 85189 & 0.02930354 & 6.45 \\
\hline & 7.29 & 28.24161923 & \\
\hline om & 5.96304327 & 0.47625659 & 7.87 \\
\hline $\mathrm{m}$ & 1.5431 & 13.88264064 & 1.300 \\
\hline om & 2.87 & 14.83473614 & \\
\hline om & 6.28 & -0.03037579 & 2.43 \\
\hline om & 6.9 & 1822777 & \\
\hline om & 8057 & 796379 & 2.15 \\
\hline om & 1.86 & 41449 & \\
\hline om & 8.3 & 4592 & 4.0 \\
\hline om & 4.92 & 3412 & \\
\hline $\mathrm{m}$ & 0.5 & 9975 & \\
\hline $\mathrm{m}$ & & 9190 & \\
\hline & & & \\
\hline om & 4.7 & 1390 & \\
\hline & & & \\
\hline & 4.0 & 908 & \\
\hline & & & \\
\hline om & 5.1 & 5645 & \\
\hline om & & & \\
\hline om & 3.6 & 0654 & 7.9 \\
\hline om & 6.2 & 67731 & \\
\hline om & 7.01 & 16518 & 6.65 \\
\hline om & 2.5 & 2897 & \\
\hline om & 2989 & 4985 & \\
\hline om & 7.22 & 7032 & 2.4 \\
\hline & 6.03 & 26.51228319 & \\
\hline $\mathrm{m}$ & 20 & 0479 & \\
\hline & & & \\
\hline $\mathrm{m}$ & 5640 & 12.29352701 & \\
\hline & & & \\
\hline om & 1.20 & 5174482 & 1.5 \\
\hline om & 3.21 & 5879 & 6.12 \\
\hline & 6.54567306 & 12.97151196 & \\
\hline $\mathrm{m}$ & 6.7 & 15. & 7.0 \\
\hline & 2.29002769 & 27.32983158 & 2.118 \\
\hline & 2.12 & 1.38756813 & 6.70 \\
\hline & 8.26 & 0.41259656 & $3.74920502 \mathrm{C}$ \\
\hline & 4.9 & 9278 & 8.3 \\
\hline & 0.57 & 14.77069085 & 0.84 \\
\hline & 3.8 & 13.94668612 & 5.42 \\
\hline & 8.525 & 12.99950216 & 3.85 \\
\hline & 4.72957879 & 15.71828023 & 8.4393 \\
\hline & & & \\
\hline & 4.10752951 & 1.35976299 & $5.32841802 \mathrm{C}$ \\
\hline & 8.23386318 & & 3.35071 \\
\hline & 5.02163167 & 26.97207366 & 7.94048 \\
\hline & 0.60317854 & 16.10391105 & 1.2387 \\
\hline & 3.81412331 & 12.61346705 & 5.8278 \\
\hline & 7.91202062 & 10.84156025 & 2.70645969 \\
\hline & 5.34345714 & 17.87623440 & 7.29574 \\
\hline & 0.92392651 & 25.19974108 & 1.88354852 \\
\hline & 3.493 & & 6.472052 \\
\hline om & 7.18076290 & 3.63555205 & 2.01130841 \\
\hline
\end{tabular}




$\begin{array}{llll}\text { atom } & 6.07469530 & 25.08229577 & 6.60102026 \mathrm{C} \\ \text { atom } & 1.65603679 & 17.99379106 & 2.57824597 \mathrm{C} \\ \text { atom } & 2.76124176 & 10.72357865 & 7.16718464 \mathrm{C}\end{array}$

$(\boldsymbol{S} \text {-MBA })_{2} \mathbf{P b}_{1-x} \mathbf{S n}_{x} \mathbf{I}_{4}(\mathrm{x}=0.25) \quad \mathbf{c}(2 \times 2)$ unit cell, PBE+TS relaxed with "tight"
default setting

$\begin{array}{llll}\text { lattice_vector } & 8.81041156 & -0.00268405 & -0.00109986 \\ \text { lattice_vector } & 0.00165906 & 28.79305399 & -0.00097985\end{array}$

lattice vector $\quad-0.00096978 \quad-0.00261020 \quad 9.16462549$

atom $\begin{array}{lll}5.39625406 & 28.75120688 & -0.00551463 \mathrm{Sn}\end{array}$

$\begin{array}{llll}\text { atom } & 7.88656652 & 14.35327975 & 9.09685099 \mathrm{~Pb}\end{array}$

atom $\quad 3.48249581 \quad 14.43176522 \quad 4.65030140 \mathrm{~Pb}$

atom $\quad 0.98716303 \quad 0.03297217 \quad 4.51364066 \mathrm{~Pb}$

$\begin{array}{llll}\text { atom } & 3.72985717 & 0.02106828 & 2.90428293 \mathrm{I}\end{array}$

atom $\quad 0.66502151 \quad 14.42369241 \quad 6.22919403 \mathrm{I}$

atom $\quad \begin{array}{llll}5.06702215 & 14.36474991 & 7.52132980 \mathrm{I}\end{array}$

$\begin{array}{llll}\text { atom } & 8.09106186 & 28.76204611 & 1.61788831 \mathrm{I}\end{array}$

$\begin{array}{llll}\text { atom } & 2.62556957 & 28.78741887 & 7.37536562 \mathrm{I}\end{array}$

$\begin{array}{llll}\text { atom } & 1.80706285 & 14.38964553 & 1.75984403 \mathrm{I}\end{array}$

$\begin{array}{llll}\text { atom } & 6.21029676 & 14.39909943 & 2.82101269 \mathrm{I}\end{array}$

$\begin{array}{llll}\text { atom } & 7.00112985 & -0.00757011 & 6.39207168 \mathrm{I}\end{array}$

$\begin{array}{llll}\text { atom } & 5.47055053 & 25.59863178 & 8.90111975 \mathrm{I}\end{array}$

$\begin{array}{llll}\text { atom } & 7.74483226 & 11.16210548 & 0.26046683 \mathrm{I}\end{array}$

atom $\quad 3.34002875 \quad 17.62619137 \quad 4.32209251 \mathrm{I}$

$\begin{array}{llll}\text { atom } & 1.06167613 & 3.22638894 & 4.84007129 \mathrm{I}\end{array}$

atom $\quad 5.51194094 \quad 3.12914605 \quad 8.88864198 \mathrm{I}$

$\begin{array}{llll}\text { atom } & 7.70367628 & 17.56382821 & 0.27281922 \mathrm{I}\end{array}$

atom $\quad 3.29541376 \quad 11.22448761 \quad 4.31076944 \mathrm{I}$

atom $\quad 1.10475044 \quad 25.61565954 \quad 4.85522156 \mathrm{I}$

$\begin{array}{llll}\text { atom } & 8.46317371 & 7.20139286 & 9.02479195 \mathrm{C}\end{array}$

atom $\quad \begin{array}{llll}4.75352509 & 21.61986733 & 0.14057610 \mathrm{C}\end{array}$

$\begin{array}{llll}\text { atom } & 0.34468114 & 7.17931175 & 4.44287984 \mathrm{C}\end{array}$

$\begin{array}{llll}\text { atom } & 4.06338986 & 21.58022615 & 4.72538216 \mathrm{C}\end{array}$

$\begin{array}{llll}\text { atom } & 0.65755189 & 7.65119846 & 8.17315597 \mathrm{C}\end{array}$

$\begin{array}{llll}\text { atom } & 3.74821278 & 22.06661656 & 0.99285728 \mathrm{C}\end{array}$

$\begin{array}{llll}\text { atom } & 8.15020199 & 6.73049385 & 3.58862855 \mathrm{C}\end{array}$

atom $\quad 5.06879423 \quad 21.13738233 \quad 5.57962355 \mathrm{C}$

$\begin{array}{llll}\text { atom } & 0.59016487 & 4.96995287 & 8.96328901 \mathrm{C}\end{array}$

$\begin{array}{llll}\text { atom } & 3.81821934 & 19.38459545 & 0.20439315 \mathrm{C}\end{array}$

$\begin{array}{llll}\text { atom } & 8.21884061 & 9.41127860 & 4.38047142 \mathrm{C}\end{array}$

$\begin{array}{llll}\text { atom } & 4.99797502 & 23.81596936 & 4.78033993 \mathrm{C}\end{array}$

$\begin{array}{llll}\text { atom } & 8.42334726 & 5.86477585 & 0.23840662 \mathrm{C}\end{array}$

$\begin{array}{llll}\text { atom } & 4.79441132 & 20.27778442 & 8.92761426 \mathrm{C}\end{array}$

$\begin{array}{llll}\text { atom } & 0.38577725 & 8.51814497 & 4.82238428 \mathrm{C}\end{array}$

$\begin{array}{llll}\text { atom } & 4.02136157 & 22.91792076 & 4.34226235 \mathrm{C}\end{array}$

$\begin{array}{llll}\text { atom } & 1.62874627 & 6.76494316 & 7.71078309 \mathrm{C}\end{array}$

$\begin{array}{llll}\text { atom } & 2.77811092 & 21.17946802 & 1.45595908 \mathrm{C}\end{array}$

$\begin{array}{llll}\text { atom } & 7.17944004 & 7.61748715 & 3.12684254 \mathrm{C}\end{array}$

$\begin{array}{llll}\text { atom } & 6.03837873 & 22.02674326 & 6.03945350 \mathrm{C}\end{array}$

$\begin{array}{llll}\text { atom } & 1.60133609 & 5.43194277 & 8.10979022 \mathrm{C}\end{array}$

$\begin{array}{llll}\text { atom } & 2.80692086 & 19.84617500 & 1.05804975 \mathrm{C}\end{array}$

$\begin{array}{llll}\text { atom } \quad 7.20767797 & 8.95030652 & 3.52638572 \mathrm{C}\end{array}$

$\begin{array}{llll}\text { atom } & 6.00893265 & 23.35860095 & 5.63655281 \mathrm{C}\end{array}$

atom $\quad 5.41681880 \quad 5.11455894 \quad 5.31383209 \mathrm{C}$

$\begin{array}{llll}\text { atom } & 7.79738524 & 19.51943267 & 3.85010114 \mathrm{C}\end{array}$

$\begin{array}{llll}\text { atom } & 3.39406428 & 9.26072702 & 0.73397876 \mathrm{C}\end{array}$

$\begin{array}{llll}\text { atom } & 1.01486387 & 23.67278399 & 8.43536151 \mathrm{C}\end{array}$

atom $\quad 5.87772091 \quad 3.38499823 \quad 3.49757946 \mathrm{C}$

$\begin{array}{llll}\text { atom } & 7.33412142 & 17.78540680 & 5.66172963 \mathrm{C}\end{array}$

$\begin{array}{llll}\text { atom } & 2.93106030 & 10.99210627 & 8.08626278 \mathrm{C}\end{array}$

$\begin{array}{llll}\text { atom } & 1.47370431 & 25.41126666 & 1.08287644 \mathrm{C}\end{array}$

$\begin{array}{llll}\text { atom } & 5.70118897 & 3.66544591 & 4.98245847 \mathrm{C}\end{array}$

atom $\quad \begin{array}{llll}7.51022920 & 18.07012502 & 4.17768303 \mathrm{C}\end{array}$

$\begin{array}{llll}\text { atom } & 3.10661314 & 10.71007210 & 0.40595733 \mathrm{C}\end{array}$

$\begin{array}{llll}\text { atom } & 1.30252137 & 25.12209361 & 8.76312932 \mathrm{C}\end{array}$

$\begin{array}{llll}\text { atom } & 6.30327454 & 5.82162245 & 6.13450216 \mathrm{C}\end{array}$ 


\begin{tabular}{|c|c|c|c|}
\hline atom & 6.91144637 & 20.22942043 & $3.03139810 \mathrm{C}$ \\
\hline ator & 2.50854143 & 8.55110941 & $1.55358564 \mathrm{C}$ \\
\hline ton & 1.89990613 & 22.96251814 & $7.61584418 \mathrm{C}$ \\
\hline $\mathrm{om}$ & 4.32665501 & 5.79101033 & $4.75129855 \mathrm{C}$ \\
\hline atom & 0.07889425 & 20.19559816 & $4.41382621 \mathrm{C}$ \\
\hline atom & 4.48472172 & 8.58636146 & $0.16993198 \mathrm{C}$ \\
\hline $\mathrm{m}$ & 8.73311673 & 22.99704035 & $8.99694812 \mathrm{C}$ \\
\hline om & 6.10659485 & 7.17788760 & $6.38387305 \mathrm{C}$ \\
\hline om & 7.11079806 & 21.58534955 & $2.78298177 \mathrm{C}$ \\
\hline om & 2.70677002 & 7.19479704 & $1.80103623 \mathrm{C}$ \\
\hline $\mathrm{m}$ & 1.69955502 & 21.60683765 & $7.36669184 \mathrm{C}$ \\
\hline om & 4.13326753 & 7.14831578 & $4.99958655 \mathrm{C}$ \\
\hline om & 0.27466217 & 21.55283154 & $4.16688364 \mathrm{C}$ \\
\hline $\mathrm{m}$ & 4.67929485 & 7.22865725 & $0.41573435 \mathrm{C}$ \\
\hline om & 8.53669714 & 21.63986501 & $8.74981795 \mathrm{C}$ \\
\hline at & 5.02151871 & 7.84217914 & $5.81900564 \mathrm{C}$ \\
\hline & 8.19780719 & 22.24666864 & $3.34758304 \mathrm{C}$ \\
\hline $\mathrm{m}$ & 3.79225580 & 6.53249758 & $1.23448990 \mathrm{C}$ \\
\hline $\mathrm{m}$ & 0.61258565 & 20.94583712 & $7.93180756 \mathrm{C}$ \\
\hline $\mathrm{m}$ & 0.55965175 & & $0.28912255 \mathrm{C}$ \\
\hline $\mathrm{m}$ & 3.84739706 & 17.9 & $95 \mathrm{C}$ \\
\hline 11 & 8.24980376 & 10.83 & $38 \mathrm{C}$ \\
\hline $\mathrm{m}$ & 4.96513708 & 25.24247701 & $4.28256204 \mathrm{C}$ \\
\hline $\mathrm{m}$ & 1.86772297 & 3.04724170 & $0.87614549 \mathrm{C}$ \\
\hline & 2.53767923 & 84721 & $204 \mathrm{C}$ \\
\hline$n$ & 6.94024864 & 11.3 & $749 \mathrm{C}$ \\
\hline$n$ & 4934 & 25.7 & $6279 \mathrm{C}$ \\
\hline $\mathrm{m}$ & 7.72909607 & 7.9 & $03 \mathrm{H}$ \\
\hline $\mathrm{m}$ & 5.48703352 & 22.32131003 & $8.91769990 \mathrm{H}$ \\
\hline $\mathrm{m}$ & 1.07945739 & 6.47509235 & $4.82936592 \mathrm{H}$ \\
\hline $\mathrm{m}$ & 3.3292 & $20.8^{\prime}$ & $123 \mathrm{H}$ \\
\hline IIII & 0.68484170 & 8.70228754 & $7.89169713 \mathrm{H}$ \\
\hline$n$ & 3.71929213 & 23.11798064 & $690 \mathrm{H}$ \\
\hline 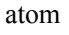 & 8.12 & 5.6 & $22 \mathrm{H}$ \\
\hline $\mathrm{m}$ & 5.09803860 & $20.08^{\prime}$ & $276 \mathrm{H}$ \\
\hline $\mathrm{m}$ & 7.62895981 & 5.50363981 & $0.89020176 \mathrm{H}$ \\
\hline $\mathrm{m}$ & 5.58887613 & 19.91 & $7726 \mathrm{H}$ \\
\hline$n$ & 1.17993394 & 8.87822472 & $5.47496675 \mathrm{H}$ \\
\hline $\mathrm{n}$ & 3.22716979 & 23.27544602 & $3.68842647 \mathrm{H}$ \\
\hline & 2.43 & & $32 \mathrm{H}$ \\
\hline 1 & 1.968 & 21.5 & $89 \mathrm{H}$ \\
\hline $\mathrm{m}$ & 6.37046509 & 7.26917248 & $2.48772483 \mathrm{H}$ \\
\hline $\mathrm{m}$ & 6.84758272 & 21.68091658 & $6.67960736 \mathrm{H}$ \\
\hline $\mathrm{m}$ & 2.39400017 & 4.76290546 & $7.77388882 \mathrm{H}$ \\
\hline $\mathrm{m}$ & 2.01488624 & 19.17677162 & $1.39467261 \mathrm{H}$ \\
\hline $\mathrm{n}$ & 6.41549093 & 9.61988212 & $3.19051139 \mathrm{H}$ \\
\hline & 6.80026837 & 24.02995024 & $5.97090380 \mathrm{H}$ \\
\hline 1 & 3.65 & 3.0 & 5.2 \\
\hline $\mathrm{m}$ & 0.74572481 & 17.41372616 & $3.94968996 \mathrm{H}$ \\
\hline & 5.15166757 & 11.37155829 & $0.63646088 \mathrm{H}$ \\
\hline $\mathrm{m}$ & 8.06844871 & 25.77752567 & $8.52489229 \mathrm{H}$ \\
\hline $\mathrm{n}$ & 4.63618354 & 2.81102723 & $6.58126992 \mathrm{H}$ \\
\hline 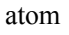 & 8.57681201 & 17.22712297 & $2.57464703 \mathrm{H}$ \\
\hline & 4.17086574 & 11.55526949 & $2.00970163 \mathrm{H}$ \\
\hline & 0.24214182 & 25.96586660 & $7.15598349 \mathrm{H}$ \\
\hline & 4.77754365 & 1.78448269 & $5.26455410 \mathrm{H}$ \\
\hline & 8.41695733 & 16.18559779 & $3.87646020 \mathrm{H}$ \\
\hline & 4.01102405 & 12.59568161 & $0.70720553 \mathrm{H}$ \\
\hline $\mathrm{n}$ & 0.39414985 & 27.00640221 & $8.46053872 \mathrm{H}$ \\
\hline & 6.18968246 & 2.34819844 & $3.32001367 \mathrm{H}$ \\
\hline & 7.01835954 & 16.74910248 & $5.83558480 \mathrm{H}$ \\
\hline & 2.61430577 & 12.02808657 & $7.91220138 \mathrm{H}$ \\
\hline & 1.79266289 & 26.44673054 & $1.25608797 \mathrm{H}$ \\
\hline & 6.65690329 & 4.04224791 & $3.09929568 \mathrm{H}$ \\
\hline & 6.55758168 & 18.44412386 & $6.06271626 \mathrm{H}$ \\
\hline & 2.15600837 & 10.33248509 & $7.68389907 \mathrm{H}$ \\
\hline & 2.24600468 & 24.75083417 & $1.48916798 \mathrm{H}$ \\
\hline & 4.95784270 & 3.56801079 & $2.92910434 \mathrm{H}$ \\
\hline & 8.25499246 & 17.96360466 & $6.23014563 \mathrm{H}$ \\
\hline & 3.85295816 & 10.81527205 & $7.51909025 \mathrm{H}$ \\
\hline
\end{tabular}




\begin{tabular}{|c|c|c|c|}
\hline atom & 0.54952029 & 25.23712826 & $\mathrm{H}$ \\
\hline atom & 6.60848718 & 3.35537535 & $5.51821250 \mathrm{H}$ \\
\hline om & 6.60145772 & 17.76400904 & $3.64212557 \mathrm{H}$ \\
\hline & 2.19702280 & 11.01557239 & 0.94049090 \\
\hline $\mathrm{m}$ & 2.21353149 & 25.42709381 & $8.23073168 \mathrm{H}$ \\
\hline & 7.17143147 & 5.31635139 & 6.5579 \\
\hline & 6.04179717 & 19.72637391 & 2.60 \\
\hline & 1.63989252 & 9.05474935 & $1.97793387 \mathrm{H}$ \\
\hline & 2.76965256 & 23.46517949 & 7.19 \\
\hline om & 3.62734871 & 5.26408471 & $4.10142550 \mathrm{H}$ \\
\hline & 0.77782792 & 19.66647760 & $3977 \mathrm{H}$ \\
\hline om & 5.18244627 & 9.11252898 & $8.68555097 \mathrm{H}$ \\
\hline om & 8.03571653 & 23.52897475 & 0.48 \\
\hline om & 6.82654056 & 8637 & 6.99 \\
\hline om & 6.39142302 & 22.12784006 & $374498 \mathrm{H}$ \\
\hline om & 1.98757707 & 8307 & 2.41 \\
\hline om & 2.41814113 & 4001 & 6.7 \\
\hline om & 3.31103596 & & 4.52 \\
\hline om & 1.09824307 & 3083 & 4.6 \\
\hline om & 5.50164804 & 6.70 & -0.0 \\
\hline om & 7.713 & 55090 & 9.2 \\
\hline om & 4.873 & & 5.98 \\
\hline om & $8.34^{\prime}$ & 23.3 & \\
\hline om & 3.94 & 5.4 & 1.3 \\
\hline & & & \\
\hline om & 8.57 & 3.4 & 1.0 \\
\hline om & & & \\
\hline om & 0.23 & 10.9 & \\
\hline om & & & \\
\hline om & 0.582 & 2.8 & 7.4 \\
\hline om & 3.83 & 17.2 & \\
\hline om & 8.23 & 11.5 & 2.8 \\
\hline om & 498 & 669 & \\
\hline om & 7.892 & 2.7 & 8.1 \\
\hline om & 5.32 & 17.1 & \\
\hline om & 0.918 & 11.6 & \\
\hline om & 3.47 & 26. & \\
\hline om & 0.279 & 1.6 & 8.5 \\
\hline & 4.11 & 16.0 & \\
\hline om & 8.520 & 12.7 & \\
\hline om & 4.658 & 27.1 & \\
\hline & & & \\
\hline om & 1.712 & 2778 & \\
\hline om & 4022 & & \\
\hline om & 7.09523634 & 25.7 & \\
\hline om & 1.773 & 2.0 & 1.2 \\
\hline & 2.63008532 & 16.4 & \\
\hline om & 7.03 & 12.3 & 5.8 \\
\hline om & 6.17780862 & 26.76 & \\
\hline & 2.13 & 3.6 & $722 \mathrm{H}$ \\
\hline & 2.27552817 & 18.0 & \\
\hline & 6.677 & 10.7 & 7191 \\
\hline & 6.549 & 25.10 & \\
\hline & 4.612 & 2.76752550 & $5.53519723 \mathrm{~N}$ \\
\hline & 8.59533861 & 17.17048369 & $3.62026553 \mathrm{~N}$ \\
\hline & 4.19031083 & 11.61110726 & 0.96403180 \\
\hline & & 26.02165324 & \\
\hline & 0.11351990 & 2.62682350 & $8.32733543 \mathrm{~N}$ \\
\hline & 4.29291751 & 17.03777777 & \\
\hline & 8.69606234 & 11.75616512 & \\
\hline tom & 4.50877826 & 26.16128716 & 5.40036585 \\
\hline
\end{tabular}

\section{$(S-M B A)_{2} \mathbf{P b}_{1-x} \mathrm{Sn}_{x} \mathbf{I}_{4}(\mathrm{x}=0.75) \quad \mathbf{c}(\mathbf{2} \times \mathbf{2})$ unit ce11, $\mathrm{PBE}+\mathrm{TS}$ relaxed with "tight" default setting}

lattice_vector $\quad 8.81587698 \quad-0.00102284 \quad-0.00134347$ lattice_vector $\quad-0.00431744 \quad 28.76204528 \quad-0.00259170$ 


\begin{tabular}{|c|c|c|c|}
\hline lattice & vector & -0 . & 492 \\
\hline atom & 3.18592766 & 7.22359910 & $2.22823764 \mathrm{~Pb}$ \\
\hline atom & 1.13498501 & 21.52994583 & $6.91475334 \mathrm{Sn}$ \\
\hline atom & 5.54579666 & 21.61095923 & $2.25244635 \mathrm{Sn}$ \\
\hline atom & 7.59674046 & 7.14934247 & $6.86757921 \mathrm{Sn}$ \\
\hline atom & 3.29716979 & 4.01247711 & $2.56802010 \mathrm{I}$ \\
\hline atom & 1.10765079 & 24.71481693 & $7.15797400 \mathrm{I}$ \\
\hline atom & 5.51503283 & 18.42410396 & $2.01082812 \mathrm{I}$ \\
\hline atom & 7.70866549 & 10.32265073 & $6.59394170 \mathrm{I}$ \\
\hline atom & 3.25216324 & 10.41738252 & $2.54588808 \mathrm{I}$ \\
\hline atom & 1.15158183 & 18.36889288 & $7.13505156 \mathrm{I}$ \\
\hline atom & 5.55946998 & 24.77049654 & $2.03287357 \mathrm{I}$ \\
\hline atom & 7.66475028 & 3.99935494 & $6.61337169 \mathrm{I}$ \\
\hline atom & 5.92336586 & 7.21093258 & $0.61721182 \mathrm{I}$ \\
\hline atom & 7.36469545 & 21.53402908 & $5.31304648 \mathrm{I}$ \\
\hline atom & 2.96130889 & 21.60631704 & $3.85821467 \mathrm{I}$ \\
\hline atom & 1.46732997 & 7.16261007 & $8.49060932 \mathrm{I}$ \\
\hline atom & 4.81315967 & 7.18642146 & $5.08343240 \mathrm{I}$ \\
\hline atom & 8.45676592 & 21.56232961 & $0.44324697 \mathrm{I}$ \\
\hline atom & 4.04882946 & 21.57639807 & $8.72137204 \mathrm{I}$ \\
\hline atom & 0.37802050 & 7.18558026 & $4.10333415 \mathrm{I}$ \\
\hline atom & 6.70230688 & 4.55571376 & $3.11193947 \mathrm{~N}$ \\
\hline atom & 6.52135856 & 24.21529517 & $7.71153057 \mathrm{~N}$ \\
\hline atom & 2.11353944 & 18.93164644 & $1.45753329 \mathrm{~N}$ \\
\hline atom & 2.30610845 & 9.82234139 & $6.03368861 \mathrm{~N}$ \\
\hline atom & 6.80208673 & 9.96057565 & $3.24060491 \mathrm{~N}$ \\
\hline atom & 6.42257890 & 18.78343565 & $7.82977662 \mathrm{~N}$ \\
\hline atom & 2.01401878 & 24.34912909 & $1.33979741 \mathrm{~N}$ \\
\hline atom & 2.40753310 & 4.41885943 & $5.91445497 \mathrm{~N}$ \\
\hline atom & 6.85008216 & 5.54580189 & $2.85514536 \mathrm{H}$ \\
\hline atom & 6.38513646 & 23.22222552 & $7.45854104 \mathrm{H}$ \\
\hline atom & 1.97920145 & 19.92522766 & $1.70938721 \mathrm{H}$ \\
\hline atom & 2.47238217 & 8.83172980 & $6.27596162 \mathrm{H}$ \\
\hline atom & 7.17648310 & 4.37701617 & $4.02168240 \mathrm{H}$ \\
\hline atom & 6.04348154 & 24.39509842 & $8.61921237 \mathrm{H}$ \\
\hline atom & 1.63528158 & 18.75153184 & $0.55020358 \mathrm{H}$ \\
\hline atom & 2.77381417 & 10.02037569 & $5.12420008 \mathrm{H}$ \\
\hline atom & 5.67317284 & 4.42814718 & $3.24906825 \mathrm{H}$ \\
\hline atom & 7.54941773 & 24.35149504 & $7.84931356 \mathrm{H}$ \\
\hline atom & 3.14125148 & 18.79307502 & $1.31958679 \mathrm{H}$ \\
\hline atom & 1.27462998 & 9.93447141 & $5.90157407 \mathrm{H}$ \\
\hline atom & 5.84121838 & 10.21498565 & $2.92237521 \mathrm{H}$ \\
\hline atom & 7.38278785 & 18.51673679 & $7.51957108 \mathrm{H}$ \\
\hline atom & 2.97341682 & 24.61716598 & $1.65110626 \mathrm{H}$ \\
\hline atom & 1.44545254 & 4.17691557 & $6.23792573 \mathrm{H}$ \\
\hline atom & 6.82812923 & 10.00368331 & $4.28650928 \mathrm{H}$ \\
\hline atom & 6.38913234 & 18.74647134 & $8.87583220 \mathrm{H}$ \\
\hline atom & 1.98178503 & 24.38726201 & $0.29377962 \mathrm{H}$ \\
\hline atom & 2.43139647 & 4.36184851 & $4.86902039 \mathrm{H}$ \\
\hline atom & 6.96501790 & 8.97740063 & $2.96891185 \mathrm{H}$ \\
\hline atom & 6.27669887 & 19.76672634 & $7.54786725 \mathrm{H}$ \\
\hline atom & 1.86939796 & 23.36539275 & $1.62066896 \mathrm{H}$ \\
\hline atom & 2.58044400 & 5.40411189 & $6.17238968 \mathrm{H}$ \\
\hline atom & 7.07899367 & 16.10120951 & $3.69486448 \mathrm{H}$ \\
\hline atom & 6.13094999 & 12.64852991 & $8.27866609 \mathrm{H}$ \\
\hline atom & 1.72639500 & 1.71849229 & $0.88891725 \mathrm{H}$ \\
\hline atom & 2.66086835 & 27.03923743 & $5.47671112 \mathrm{H}$ \\
\hline atom & 5.51063642 & 14.87694527 & $2.23286893 \mathrm{H}$ \\
\hline atom & 7.69959673 & 13.87029280 & $6.81519697 \mathrm{H}$ \\
\hline atom & 3.29561665 & 0.49674341 & $2.35161911 \mathrm{H}$ \\
\hline atom & 1.09723955 & 28.26908050 & $6.93861998 \mathrm{H}$ \\
\hline atom & 7.30073353 & 27.24405059 & $3.57376087 \mathrm{H}$ \\
\hline atom & 5.91023491 & 1.52130281 & $8.15461498 \mathrm{H}$ \\
\hline atom & 1.49997432 & 12.86781037 & $1.01138397 \mathrm{H}$ \\
\hline atom & 2.88639073 & 15.89796496 & $5.60231204 \mathrm{H}$ \\
\hline atom & 5.52882225 & 28.02806683 & $2.05121588 \mathrm{H}$ \\
\hline atom & 7.68466523 & 0.73472853 & $6.63705143 \mathrm{H}$ \\
\hline atom & 3.27313344 & 13.65311601 & $2.53055697 \mathrm{H}$ \\
\hline atom & 1.11741388 & 15.10450639 & $7.12278586 \mathrm{H}$ \\
\hline atom & 0.21301875 & 14.90560073 & $4.70213152 \mathrm{H}$ \\
\hline
\end{tabular}




\begin{tabular}{|c|c|c|c|}
\hline atom & 4.18386653 & 13.84962676 & $0.11621975 \mathrm{H}$ \\
\hline atom & 8.58989709 & 0.51745147 & $9.05416212 \mathrm{H}$ \\
\hline atom & 4.61376537 & 28.22953723 & $4.46842900 \mathrm{H}$ \\
\hline atom & 8.36917589 & 5.16555895 & $1.04320045 \mathrm{H}$ \\
\hline atom & 4.86024479 & 23.58139846 & $5.64492890 \mathrm{H}$ \\
\hline atom & 0.45134749 & 19.56945703 & $3.52236693 \mathrm{H}$ \\
\hline atom & 3.96379063 & 9.20497904 & $8.10665064 \mathrm{H}$ \\
\hline atom & 8.74465282 & 3.50667352 & $0.56201169 \mathrm{H}$ \\
\hline atom & 4.47562211 & 25.23466360 & $5.15245308 \mathrm{H}$ \\
\hline atom & 0.06596239 & 17.91709228 & $4.01710684 \mathrm{H}$ \\
\hline atom & 4.32291816 & 10.86156161 & $8.60825996 \mathrm{H}$ \\
\hline atom & 0.47420318 & 4.11091730 & $2.14029861 \mathrm{H}$ \\
\hline atom & 3.93203705 & 24.63786191 & $6.73253253 \mathrm{H}$ \\
\hline atom & 8.33882026 & 18.51085436 & $2.43475207 \mathrm{H}$ \\
\hline atom & 4.88658854 & 10.27642255 & $7.03027663 \mathrm{H}$ \\
\hline atom & 8.37615701 & 9.54173814 & $1.02126573 \mathrm{H}$ \\
\hline atom & 4.84627222 & 19.21625662 & $5.61198777 \mathrm{H}$ \\
\hline atom & 0.43593906 & 23.91339092 & $3.55625975 \mathrm{H}$ \\
\hline atom & 3.97654570 & 4.84311673 & $8.13695476 \mathrm{H}$ \\
\hline atom & 8.84080944 & 11.23647633 & $0.80013664 \mathrm{H}$ \\
\hline atom & 4.37849997 & 17.52340677 & $5.38553548 \mathrm{H}$ \\
\hline atom & -0.03559444 & 25.60527126 & $3.78165866 \mathrm{H}$ \\
\hline atom & 4.42730697 & 3.14677341 & $8.37215123 \mathrm{H}$ \\
\hline atom & 7.14188531 & 10.76035545 & $0.63442111 \mathrm{H}$ \\
\hline atom & 6.07840997 & 17.99609309 & $5.22142910 \mathrm{H}$ \\
\hline atom & 1.66511819 & 25.13599522 & $3.94854123 \mathrm{H}$ \\
\hline atom & 2.73103998 & 3.63525328 & $8.52550903 \mathrm{H}$ \\
\hline atom & 5.42630672 & 1.66709678 & $1.40211044 \mathrm{H}$ \\
\hline atom & 7.79066178 & 27.09345151 & $5.98986804 \mathrm{H}$ \\
\hline atom & 3.38102567 & 16.05524830 & $3.17992537 \mathrm{H}$ \\
\hline atom & 1.01456388 & 12.70000651 & $7.76404644 \mathrm{H}$ \\
\hline atom & 5.81929852 & 12.46240245 & $1.80934071 \mathrm{H}$ \\
\hline atom & 7.39488830 & 16.28574442 & $6.39294528 \mathrm{H}$ \\
\hline atom & 2.98489867 & 26.84424010 & $2.77425603 \mathrm{H}$ \\
\hline atom & 1.41543618 & 1.92179250 & $7.36083932 \mathrm{H}$ \\
\hline atom & 0.23541524 & 0.08040015 & $4.39242801 \mathrm{H}$ \\
\hline atom & 4.15894605 & 28.68707645 & $8.96802795 \mathrm{H}$ \\
\hline atom & 8.56662729 & 14.46225561 & $0.19762449 \mathrm{H}$ \\
\hline atom & 4.63618747 & 14.30751023 & $4.78084108 \mathrm{H}$ \\
\hline atom & 0.55050316 & 12.50333340 & $4.26283074 \mathrm{H}$ \\
\hline atom & 3.84837383 & 16.25035812 & $8.84615247 \mathrm{H}$ \\
\hline atom & 8.25105958 & 26.87952088 & $0.32433843 \mathrm{H}$ \\
\hline atom & 4.96208650 & 1.86908828 & $4.90813483 \mathrm{H}$ \\
\hline atom & 8.99940359 & 2.42805415 & $3.68220229 \mathrm{H}$ \\
\hline atom & 4.21317660 & 26.33652531 & $8.26460705 \mathrm{H}$ \\
\hline atom & -0.19705303 & 16.81304126 & $0.90644668 \mathrm{H}$ \\
\hline atom & 4.58970622 & 11.95614617 & $5.48110392 \mathrm{H}$ \\
\hline atom & 8.79898738 & 10.54536728 & $3.21911591 \mathrm{H}$ \\
\hline atom & 4.42211276 & 18.20915069 & $7.80691946 \mathrm{H}$ \\
\hline atom & 0.01260744 & 24.92030474 & $1.36091451 \mathrm{H}$ \\
\hline atom & 4.40190640 & 3.82744744 & $5.94843459 \mathrm{H}$ \\
\hline atom & 6.37026689 & 3.72898690 & $1.23677210 \mathrm{H}$ \\
\hline atom & 6.85109120 & 25.03035588 & $5.83127249 \mathrm{H}$ \\
\hline atom & 2.44132682 & 18.11842742 & $3.33903775 \mathrm{H}$ \\
\hline atom & 1.95726683 & 10.63589495 & $7.91211437 \mathrm{H}$ \\
\hline atom & 7.22298967 & 15.03503728 & $3.52728567 \mathrm{C}$ \\
\hline atom & 5.98773348 & 13.71464761 & $8.11047667 \mathrm{C}$ \\
\hline atom & 1.58220106 & 0.65272913 & $1.05835735 \mathrm{C}$ \\
\hline atom & 2.80802551 & 28.10515302 & $5.64314004 \mathrm{C}$ \\
\hline atom & 6.33170613 & 14.34443596 & $2.70844016 \mathrm{C}$ \\
\hline atom & 6.87949562 & 14.40396515 & $7.29110307 \mathrm{C}$ \\
\hline atom & 2.47004212 & 28.72550913 & $1.87478665 \mathrm{C}$ \\
\hline atom & 1.92348687 & 0.03704460 & $6.46444937 \mathrm{C}$ \\
\hline atom & 7.26873898 & 28.29429065 & $3.28944056 \mathrm{C}$ \\
\hline atom & 5.94288252 & 0.47039437 & $7.87280904 \mathrm{C}$ \\
\hline atom & 1.53119497 & 13.91811910 & $1.29535287 \mathrm{C}$ \\
\hline atom & 2.85715602 & 14.84660552 & $5.88282405 \mathrm{C}$ \\
\hline atom & 6.26607999 & -0.02677584 & $2.43859529 \mathrm{C}$ \\
\hline atom & 6.94715151 & 28.78998976 & $7.01948198 \mathrm{C}$ \\
\hline atom & 2.53927543 & 14.35996874 & $2.14700185 \mathrm{C}$ \\
\hline
\end{tabular}




\begin{tabular}{|c|c|c|c|}
\hline atom & 1.85160769 & 14.40020720 & \\
\hline & 8.30657498 & 14.36696766 & $4.09072527 \mathrm{C}$ \\
\hline & .90521785 & 14.38410289 & 8.674 \\
\hline & .49385834 & 28.74598227 & $1002 C$ \\
\hline & 3.89741641 & 0.00759323 & $5.08095279 \mathrm{C}$ \\
\hline & .46973320 & 4.13860113 & $2777 \mathrm{C}$ \\
\hline $\mathrm{m}$ & 4.75371865 & 24.61026366 & \\
\hline & 0.34448055 & 18.54015109 & \\
\hline & 4.05989192 & 3650143 & \\
\hline & 8.06354524 & 10.57809031 & \\
\hline & 5.15721018 & 18.1 & \\
\hline & 0.74517036 & 24.95140664 & 3.380675 \\
\hline & & & \\
\hline $\mathrm{m}$ & 6.22 & 1.31 & 2.05 \\
\hline & & 27.4 & \\
\hline $\mathrm{n}$ & 2.58 & 15. & \\
\hline & & & \\
\hline $\mathrm{m}$ & 7.19 & 2.2 & 2.4 \\
\hline & 6.0 & 26. & \\
\hline & & & \\
\hline & 2.7 & 12. & \\
\hline & & & \\
\hline & 5.6 & 16. & 7.6 \\
\hline $\mathrm{m}$ & $1.1 \mathrm{~S}$ & 26. & \\
\hline & 3.20 & 2.0 & 6.1 \\
\hline & 6.5 & 12.5 & \\
\hline & 6.6 & 15. & \\
\hline & 2.2 & 27. & $6 \mathrm{C}$ \\
\hline & 2.11 & & \\
\hline & 8.2 & 0.4 & \\
\hline & & & \\
\hline & 0.56 & & \\
\hline & & & \\
\hline & 8.49 & 13.0 & 3.839772 \\
\hline & & & \\
\hline & 0.3 & 27.3 & \\
\hline $\mathrm{m}$ & 4.0 & 1.3 & $6 \mathrm{C}$ \\
\hline & 8.20 & 1.7 & \\
\hline & 5.00 & 27.0 & $3 \mathrm{C}$ \\
\hline & 0.59 & 16.1 & \\
\hline & 3.79 & 12.6 & 5.8 \\
\hline & 7.89 & 10.8 & \\
\hline & 5.328 & 17.8 & 7.27289891 \\
\hline & 0.91836061 & 25.23580392 & 1.89602449 \\
\hline & 3.49035282 & 3.52100438 & $6.47869152 \mathrm{C}$ \\
\hline & 7.16071743 & 3.63718357 & \\
\hline & 6.05931207 & 25.12299577 & 6.58762890 \\
\hline & 1.64990992 & 18.02587828 & \\
\hline & 2.75268564 & 10.73537859 & 7.16056874 \\
\hline
\end{tabular}

\section{References}

(1) Billing, D. G.; Lemmerer, A. Synthesis and crystal structures of inorganic-organic hybrids incorporating an aromatic amine with a chiral functional group. CrystEngComm 2006, 8, 686-695.

(2) Calabrese, J.; Jones, N. L.; Harlow, R. L.; Herron, N.; Thorn, D. L.; Wang, Y. Preparation and characterization of layered lead halide compounds. Journal of the American Chemical Society 1991, 113, 2328-2330.

(3) Papavassiliou, G. C.; Koutselas, I. B.; Terzis, A.; Whangbo, M. H. Structural and electronic properties of the natural quantum-well system $(\mathrm{C} 6 \mathrm{H} 5 \mathrm{CH} 2 \mathrm{CH} 2 \mathrm{NH} 3) 2 \mathrm{Snl} 14$. Solid State Communications 1994, 91, 695-698.

(4) Mitzi, D. B. Synthesis, Crystal Structure, and Optical and Thermal Properties of (C4H9NH3)2MI4 (M = Ge, Sn, Pb). Chemistry of Materials 1996, 8, 791-800. 
(5) Mao, L.; Tsai, H.; Nie, W.; Ma, L.; Im, J.; Stoumpos, C. C.; Malliakas, C. D.; Hao, F.; Wasielewski, M. R.; Mohite, A. D.; Kanatzidis, M. G. Role of Organic Counterion in Lead- and Tin-Based Two-Dimensional Semiconducting lodide Perovskites and Application in Planar Solar Cells. Chemistry of Materials 2016, 28, 7781-7792. 